\title{
Quantitative characterization of stress concentration in the presence of closely spaced hard inclusions in two-dimensional linear elasticity*
}

\author{
Hyeonbae Kang ${ }^{\dagger} \quad$ Sanghyeon $\mathrm{Yu}^{\ddagger}$
}

April 19, 2022

\begin{abstract}
In the region between close-to-touching hard inclusions, the stress may be arbitrarily large as the inclusions get closer. The stress is represented by the gradient of a solution to the Lamé system of linear elasticity. We consider the problem of characterizing the gradient blow-up of the solution in the narrow region between two inclusions and estimating its magnitude. We introduce singular functions which are constructed in terms of nuclei of strain and hence are solutions of the Lamé system, and then show that the singular behavior of the gradient in the narrow region can be precisely captured by singular functions. As a consequence of the characterization, we are able to regain the existing upper bound on the blow-up rate of the gradient, namely, $\epsilon^{-1 / 2}$ where $\epsilon$ is the distance between two inclusions. We then show that it is in fact an optimal bound by showing that there are cases where $\epsilon^{-1 / 2}$ is also a lower bound. This work is the first to completely reveal the singular nature of the gradient blow-up in the context of the Lamé system with hard inclusions. The singular functions introduced in this paper play essential roles to overcome the difficulties in applying the methods of previous works. Main tools of this paper are the layer potential techniques and the variational principle. The variational principle can be applied because the singular functions of this paper are solutions of the Lamé system.
\end{abstract}

AMS subject classifications. 35J47, 74B05, 35B40

Key words. stress concentration, gradient blow-up, closely spaced inclusions, hard inclusion, Lamé system, linear elasticity, high contrast, optimal bound, singular functions, nuclei of strain

\section{Contents}

\section{Introduction}

${ }^{*}$ This work is supported by NRF 2016R1A2B4011304 and 2017R1A4A1014735

${ }^{\dagger}$ Department of Mathematics, Inha University, Incheon 22212, S. Korea (hbkang@inha.ac.kr)

${ }^{\ddagger}$ Seminar for Applied Mathematics, Department of Mathematics, ETH Zürich, Rämistrasse 101, CH8092 Zürich, Switzerland (sanghyeon.yu@sam.math.ethz.ch) 
2 Problem formulation and preliminaries 4

2.1 Lamé system with hard inclusions: a problem formulation . . . . . . . . . 5

2.2 Layer potentials for 2D Lamé system . . . . . . . . . . . . . . . . . 7

2.3 The exterior problem and the variational principle . . . . . . . . 9

2.4 An estimate for the free space problem . . . . . . . . . . . 13

2.5 Geometry of two inclusions . . . . . . . . . . . . . . . . 15

3 Singular functions and their properties 17

3.1 Construction of singular functions . . . . . . . . . . . . 18

3.2 Estimates of the function $\zeta \ldots \ldots \ldots \ldots \ldots$

3.3 Estimates of singular functions . . . . . . . . . . . . . 23

3.4 Approximations by singular functions . . . . . . . . . . . 32

4 Stress concentration-boundary value problem 35

4.1 Characterization of stress concentration-BVP . . . . . . . . . . 35

4.2 Preliminary estimates of boundary integrals . . . . . . . . . . . 40

4.3 Proof of Lemma $4.1 \ldots \ldots \ldots \ldots$

4.4 Estimates of integrals $\mathcal{I}_{j k}$ and $\mathcal{J}_{k}$ and proof of Proposition 4.2 . . . . . 46

4.5 Proof of Proposition $4.3 \ldots \ldots \ldots \ldots \ldots$. . . . . . . . . . . . . . . . . . . . . . . .

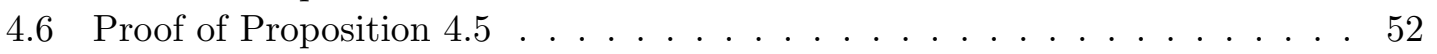

5 Stress concentration-the free space problem

6 Symmetric inclusions and optimality of the blow-up rate 58

6.1 Symmetric inclusions . . . . . . . . . . . . . . 59

6.2 Circular inclusions and optimality of the blow-up rate . . . . . . . . 60

A The Neumann-Poincaré operator and the exterior problem 64

A.1 The NP operator . . . . . . . . . . . . . . . 65

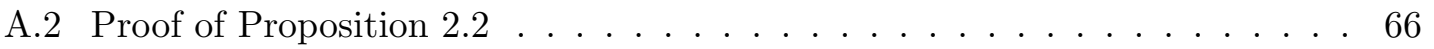

A.3 Proof of Proposition $2.1 \ldots \ldots \ldots \ldots$. . . . . . . . . . . . .

A.4 Proof of Theorem $2.5 \ldots \ldots \ldots$. . . . . . . . . . . . . . . . . . . . . .

\section{Introduction}

When two inclusions are close to touching, the physical field such as the stress or the electric field may be arbitrarily large in the narrow region between the inclusions. It is quite important to understand the field concentration precisely. Stress concentration may occur in fiber-reinforced composites where elastic inclusions are densely packed [7]. The electric field can be greatly enhanced in the conducting inclusions case. It can be utilized to achieve subwavelength imaging and sensitive spectroscopy [34].

In response to such importance there has been much progress in understanding the field concentration in the last decade or so. In the context of electrostatics (or anti-plane elasticity), the field is the gradient of a solution to the Laplace equation and the precise estimates of the gradient were obtained. It is discovered that when the conductivity of the inclusions is $\infty$, the blow-up rate of the gradient is $\epsilon^{-1 / 2}$ in two dimensions [5, 35, 
where $\epsilon$ is the distance between two inclusions, and it is $|\epsilon \ln \epsilon|^{-1}$ in three dimensions [8]. There is a long list of literature in this direction of research, e.g., [3, 4, 11, 17, 18, 26, 27, 31, 32, 36, 37. While these works are related to the estimate of the blow-up rate of the gradient, there is other direction of research to characterize the singular behavior of the gradient [1, 20, 21, 22, 29]. An explicit function, which is called a singular function, is introduced and the singular behavior of the gradient is completely characterized by this singular function. Since the singular function is closely related to this work, we include a brief discussion on it at the beginning of subsection 3.1. All the work mentioned above are related to the homogenous equation and inclusions with smooth boundaries. Recently there have been important extensions to the inhomogeneous equation [15] and inclusions with corners (the bow-tie shape) [24].

In this paper, we consider a similar problem in linear elasticity, i.e., the Lamé system. We assume two hard inclusions, which have infinite shear modulus, are presented with a small separation distance $\epsilon$. The stress is represented in terms of the gradient of a solution to the Lamé system. We are interested in the singular behavior of the stress (or the gradient) when the distance $\epsilon$ goes to zero.

Even though much progress has been made for the Laplace equation of the anti-plane elasticity as mentioned above, not much is known about the gradient blow-up in the context of the full elasticity, e.g., the Lamé system. Recently, a significant progress has been made by Bao et al [9, 10]: it is proved in [9] that $\epsilon^{-1 / 2}$ is an upper bound on the blow-up rate of the gradient in the two-dimensional Lamé system. We emphasize that there is significant difficulty in applying the methods for scalar equations to systems of equations. For instance, the maximum principle does not hold for the system. In [9] they come up with an ingenious iteration technique to overcome this difficulty and obtain the upper bound on the blow-up rate. However, it was still not known if it is also a lower bound.

The purpose of this paper is to construct singular functions for the two-dimensional Lamé system, like the one for electrostatics, and to characterize the singular behavior of the gradient using singular functions. In fact, we construct singular functions as elaborated linear combinations of nuclei of strain, and show that they capture the singular behavior of the gradient precisely. Nuclei of strain are the columns of the Kelvin matrix of the fundamental solution to the Lamé system and their variants. As a consequence of such characterization, we are able to reobtain the result of [9] with a different proof, which states that $\epsilon^{-1 / 2}$ is an upper bound on the blow-up rate of the gradient. More importantly, the characterization enables us to show that the rate $\epsilon^{-1 / 2}$ is actually optimal, optimal in the sense that there are cases where $\epsilon^{-1 / 2}$ is a lower bound on the blow-up rate. To the best of our knowledge, this work is the first to completely reveal the singular nature of the gradient blow-up in the context of the Lamé system with hard inclusions. The singular functions introduced in this paper play essential roles to overcome the difficulties in applying the methods of previous works.

We emphasize that the nuclei of strain and singular functions are solutions of the Lamé system. This has a significant implication. We heavily use the variational principle for proving the characterization of the stress concentration in section 4 , which is only possible since singular functions are solutions of the Lamé system. This makes the method of this paper significantly different from that of [9]. We include a brief comparison of two methods at the end of subsection 4.1 . 
It is worth mentioning that the singular functions constructed in this paper are applied to an important problem other than analysis of the gradient blow-up. In fact, quantitative analysis of the gradient is closely related to the computation of the effective property of densely packed composites. In [12, Beryland et al. provided the first rigorous justification of the asymptotic formula for the effective conductivity, which was found by Keller [25]. However, the corresponding formulas of Flaherty-Keller [16] for the effective elastic properties have not been rigorously proved to the best of our knowledge. Using singular functions of this paper we are able to prove the formulas in a mathematically rigorous way. We emphasize that this is possible only because singular functions are solutions of the Lamé system. We report this result in a separate paper [23].

Accurate numerical computation of the gradient in the presence of closely spaced hard inclusions is a well-known challenging problem in computational mathematics and sciences. When computing the gradient, a serious difficulty arises since a fine mesh is required to capture the gradient blow-up in the narrow region. The precise characterization of the gradient blow-up can be utilized for designing an efficient numerical scheme to compute the gradient. This was done for the conductivity case in [21]. The result of this paper may open up a way to do computation for the isotropic elasticity.

It is worth mentioning that, for the Lamé system where two inclusions are circular holes, the gradient blow-up is recently characterized by a singular function in [30]. Moreover, the optimal blow-up rate of the gradient is obtained. The holes are characterized by the vanishing traction condition on the boundary, and the blow-up rate is the same as the hard inclusion case, namely, $\epsilon^{-1 / 2}$. We emphasize that unlike the anti-plane elasticity, the hole case is not the dual problem of the hard inclusion case, and a different method is required to handle the hole case.

This paper consists of six sections including introduction and appendices. In section 2, we formulate the problem to be considered, derive some preliminary results which will be used in later sections, and describe geometry of two inclusions. In section 3 , singular functions are constructed in terms of nuclei of strain and their properties are derived for later use. Section 4 and 5 respectively deal with the problem of characterizing the stress concentration in a bounded domain and in the free space. In section 6 we consider the case when inclusions are symmetric, in particular, when inclusions are disks of the same radius, and show that $\epsilon^{-1 / 2}$ is a lower bound on the blow-up rate of the gradient when Lamé constants satisfy a certain constraint. Since each section is rather long and its subject can be viewed as independent, we include an introduction in each section. Appendices are to prove some results used in the text, especially existence and uniqueness of the solution to the exterior problem of the Lamé system and the layer potential representation of the solution to the boundary value problem and the free space problem.

Throughout this paper, we use the expression $A \lesssim B$ to imply that there is a constant $C$ independent of $\epsilon$ such that $A \leq C B$. The expression $A \approx B$ implies that both $A \lesssim B$ and $B \lesssim A$ hold.

\section{Problem formulation and preliminaries}

In this section we formulate the problem of characterizing the stress concentration. The main tools in dealing with the problem are the layer potential technique and the varia- 
tional principle. We introduce them in this section. We then consider the existence and uniqueness question of the exterior problem for the Lamé system with arbitrary Dirichlet data. The final subsection is to describe the geometry of two inclusions in a precise manner.

\subsection{Lamé system with hard inclusions: a problem formulation}

We consider two disjoint elastic inclusions $D_{1}$ and $D_{2}$ which are embedded in $\mathbb{R}^{2}$ occupied by an elastic material. We assume that $D_{1}$ and $D_{2}$ are simply connected bounded domains with $C^{4}$-smooth boundaries. We emphasize that the results of this paper are valid even if boundaries are $C^{3, \alpha}$ for some $\alpha>0$. But we assume that they are $C^{4}$ for convenience. Advantage of assuming $C^{4}$ is made clear in subsection 2.5. We also assume some convexity of the boundaries which is precisely described in the same subsection.

Let $(\lambda, \mu)$ be the pair of Lamé constants of $D^{e}:=\mathbb{R}^{2} \backslash \overline{D_{1} \cup D_{2}}$ which satisfies the strong ellipticity conditions $\mu>0$ and $\lambda+\mu>0$. Then the elasticity tensor is given by $\mathbb{C}=\left(C_{i j k l}\right)$ with

$$
C_{i j k l}=\lambda \delta_{i j} \delta_{k l}+\mu\left(\delta_{i k} \delta_{j l}+\delta_{i l} \delta_{j k}\right), \quad i, j, k, l=1,2,
$$

where $\delta_{i j}$ denotes Kronecker's delta. The Lamé operator $\mathcal{L}_{\lambda, \mu}$ of the linear isotropic elasticity is defined by

$$
\mathcal{L}_{\lambda, \mu} \mathbf{u}:=\nabla \cdot \mathbb{C} \widehat{\nabla} \mathbf{u}=\mu \Delta \mathbf{u}+(\lambda+\mu) \nabla \nabla \cdot \mathbf{u},
$$

where $\widehat{\nabla}$ denotes the symmetric gradient, namely,

$$
\widehat{\nabla} \mathbf{u}=\frac{1}{2}\left(\nabla \mathbf{u}+\nabla \mathbf{u}^{T}\right) \quad(T \text { for transpose }) .
$$

The corresponding conormal derivative $\partial_{\nu} \mathbf{u}$ on $\partial D_{j}$ is defined as

$$
\partial_{\nu} \mathbf{u}=(\mathbb{C} \widehat{\nabla} \mathbf{u}) \mathbf{n}
$$

where $\mathbf{n}$ is the outward unit normal vector to $\partial D_{j}(j=1,2)$.

Given a displacement field $\mathbf{u}=\left(u_{1}, u_{2}\right)^{T}, \widehat{\nabla} \mathbf{u}$ is the strain tensor while the stress tensor $\boldsymbol{\sigma}=\left(\sigma_{i j}\right)_{i, j=1}^{2}$ is defined to be

$$
\boldsymbol{\sigma}:=\mathbb{C} \widehat{\nabla} \mathbf{u}=\lambda \operatorname{tr}(\widehat{\nabla} \mathbf{u}) \mathbf{I}+2 \mu \widehat{\nabla} \mathbf{u}
$$

namely,

$$
\begin{aligned}
& \sigma_{11}=(\lambda+2 \mu) \partial_{1} u_{1}+\lambda \partial_{2} u_{2}, \\
& \sigma_{22}=\lambda \partial_{1} u_{1}+(\lambda+2 \mu) \partial_{2} u_{2}, \\
& \sigma_{12}=\sigma_{21}=\mu\left(\partial_{2} u_{1}+\partial_{1} u_{2}\right) .
\end{aligned}
$$

Here and throughout this paper, tr stands for the trace and $\partial_{j}$ denotes the partial derivative with respect to the $x_{j}$-variable for $j=1,2$. 
Let $\boldsymbol{\Psi}$ be the collection of all functions $\boldsymbol{\psi}$ such that $\widehat{\nabla} \boldsymbol{\psi}=0$ in $\mathbb{R}^{2}$, i.e., the threedimensional vector space spanned by the displacement fields of the rigid motions $\left\{\Psi_{j}\right\}_{j=1}^{3}$ defined as follows:

$$
\Psi_{1}(\mathbf{x})=\left[\begin{array}{l}
1 \\
0
\end{array}\right], \quad \Psi_{2}(\mathbf{x})=\left[\begin{array}{l}
0 \\
1
\end{array}\right], \quad \Psi_{3}(\mathbf{x})=\left[\begin{array}{c}
-y \\
x
\end{array}\right]
$$

Throughout this paper we denote the point $\mathbf{x}$ in $\mathbb{R}^{2}$ by either $\left(x_{1}, x_{2}\right)^{T}$ or $(x, y)^{T}$ at its convenience.

We assume $D_{1}$ and $D_{2}$ are hard inclusions. This assumption is inscribed on the boundary conditions on $\partial D_{j}$ in the following problem: Let $\Omega$ be a bounded domain in $\mathbb{R}^{2}$ containing $D_{1}$ and $D_{2}$ such that $\operatorname{dist}\left(\partial \Omega, D_{1} \cup D_{2}\right) \geq C$ for some constant $C>0$. Let us denote

$$
\widetilde{\Omega}=\Omega \backslash \overline{D_{1} \cup D_{2}} .
$$

For a given Dirichlet data $\mathbf{g}$ we consider the following problem:

$$
\begin{cases}\mathcal{L}_{\lambda, \mu} \mathbf{u}=0 & \text { in } \widetilde{\Omega}, \\ \mathbf{u}=\sum_{j=1}^{3} c_{i j} \Psi_{j}(\mathbf{x}) & \text { on } \partial D_{i}, \quad i=1,2 \\ \mathbf{u}=\mathbf{g} & \text { on } \partial \Omega,\end{cases}
$$

where the constants $c_{i j}$ are determined by the conditions

$$
\left.\int_{\partial D_{i}} \partial_{\nu} \mathbf{u}\right|_{+} \cdot \Psi_{j} d \sigma=0, \quad i=1,2, j=1,2,3 .
$$

Here and afterwards, the subscript + denotes the limit from outside $\partial D_{j}$.

$$
\text { Let }
$$

$$
\epsilon:=\operatorname{dist}\left(D_{1}, D_{2}\right)
$$

The gradient $\nabla \mathbf{u}$ of the solution $\mathbf{u}$ to 2.6 may become arbitrarily large as two inclusions get closer, namely, as $\epsilon \rightarrow 0$. The main purpose of this paper is to characterize the blow-up of $\nabla \mathbf{u}$. Roughly speaking, we show that $\mathbf{u}$ can be decomposed as

$$
\mathbf{u}=\mathbf{s}+\mathbf{b}
$$

where $\nabla \mathbf{s}$ has the main singularity of $\nabla \mathbf{u}$ while $\nabla \mathbf{b}$ is regular or less singular. So the singular behavior of $\nabla \mathbf{u}$ is characterized by that of $\nabla \mathbf{s}$. We will find $\mathbf{s}$ in an explicit form. The characterization of the gradient blow-up enables us to show that the optimal blow-up rate of $\nabla \mathbf{u}$ in terms of $\epsilon$ is $\epsilon^{-1 / 2}$. It is proved in [9] that $\epsilon^{-1 / 2}$ is an upper bound on the blow-up rate of $\nabla \mathbf{u}$ as mentioned before.

The problem in the presence of hard inclusions may be considered as the limiting problem of a high contrast elasticity problem when the shear modulus of the inclusions degenerates to infinity [9]. When the shear modulus is bounded away from zero and infinity, it is known that the gradient is bounded regardless of the distance between inclusions [28]. 
We also consider the free space problem: For a given function $\mathbf{H}$ satisfying $\mathcal{L}_{\lambda, \mu} \mathbf{H}=$ 0 in $\mathbb{R}^{2}$, the displacement field $\mathbf{u}$ satisfies

$$
\begin{cases}\mathcal{L}_{\lambda, \mu} \mathbf{u}=0 & \text { in } D^{e} \\ \mathbf{u}=\sum_{j=1}^{3} d_{i j} \Psi_{j} & \text { on } \partial D_{i}, \quad i=1,2 \\ \mathbf{u}(\mathbf{x})-\mathbf{H}(\mathbf{x})=O\left(|\mathbf{x}|^{-1}\right) & \text { as }|\mathbf{x}| \rightarrow \infty\end{cases}
$$

where the constants $d_{i j}$ are determined by the condition (2.7). We will obtain the decomposition of the form (2.9) and estimates of $\nabla \mathbf{u}$ for this problem as well.

\subsection{Layer potentials for 2D Lamé system}

The Kelvin matrix of fundamental solutions $\boldsymbol{\Gamma}=\left(\Gamma_{i j}\right)_{i, j=1}^{2}$ to the Lamé operator $\mathcal{L}_{\lambda, \mu}$ is given by

$$
\Gamma_{i j}(\mathbf{x})=\alpha_{1} \delta_{i j} \ln |\mathbf{x}|-\alpha_{2} \frac{x_{i} x_{j}}{|\mathbf{x}|^{2}}
$$

where

$$
\alpha_{1}=\frac{1}{4 \pi}\left(\frac{1}{\mu}+\frac{1}{\lambda+2 \mu}\right) \quad \text { and } \quad \alpha_{2}=\frac{1}{4 \pi}\left(\frac{1}{\mu}-\frac{1}{\lambda+2 \mu}\right) .
$$

In short, $\boldsymbol{\Gamma}$ can be expressed as

$$
\boldsymbol{\Gamma}(\mathbf{x})=\alpha_{1} \ln |\mathbf{x}-\mathbf{y}| \mathbf{I}-\alpha_{2} \mathbf{x} \otimes \nabla(\ln |\mathbf{x}|)
$$

where $\mathbf{I}$ is the identity matrix.

For a given bounded domain $D$ with $C^{2}$ boundary, the single and double layer potentials on $\partial D$ associated with the pair of Lamé parameters $(\lambda, \mu)$ are defined by

$$
\begin{aligned}
& \mathcal{S}_{\partial D}[\boldsymbol{\varphi}](\mathbf{x}):=\int_{\partial D} \boldsymbol{\Gamma}(\mathbf{x}-\mathbf{y}) \boldsymbol{\varphi}(\mathbf{y}) d \sigma(\mathbf{y}), \quad \mathbf{x} \in \mathbb{R}^{2}, \\
& \mathcal{D}_{\partial D}[\boldsymbol{\varphi}](\mathbf{x}):=\int_{\partial D} \partial_{\nu} \boldsymbol{\Gamma}(\mathbf{x}-\mathbf{y}) \boldsymbol{\varphi}(\mathbf{y}) d \sigma(\mathbf{y}), \quad \mathbf{x} \in \mathbb{R}^{2} \backslash \partial D
\end{aligned}
$$

where the conormal derivative $\partial_{\nu} \boldsymbol{\Gamma}(\mathbf{x}-\mathbf{y})$ is defined by

$$
\partial_{\nu} \boldsymbol{\Gamma}(\mathbf{x}-\mathbf{y}) \mathbf{b}=\partial_{\nu}(\boldsymbol{\Gamma}(\mathbf{x}-\mathbf{y}) \mathbf{b})
$$

for any constant vector $\mathbf{b}$.

Let $H^{1 / 2}(\partial D)$ be the usual $L^{2}$-Sobolev space of order $1 / 2$ on $\partial D$ and $H^{-1 / 2}(\partial D)$ be its dual space. With functions $\Psi_{j}$ in 2.5 we define

$$
H_{\Psi}^{-1 / 2}(\partial D):=\left\{\mathbf{f} \in H^{-1 / 2}(\partial D)^{2}: \int_{\partial D} \mathbf{f} \cdot \Psi_{j}=0, j=1,2,3\right\} .
$$

The following propositions for representations of the solutions to 2.6 and $(2.10)$ can be proved in a standard way (see, for example, [2]). We include brief proofs in Appendix. 
Proposition 2.1. Let $\mathbf{u}$ be the solution to (2.6) and let $\mathbf{f}:=\left.\partial_{\nu} \mathbf{u}\right|_{-}$on $\partial \Omega$. Define

$$
\mathbf{H}_{\Omega}(\mathbf{x})=-\mathcal{S}_{\partial \Omega}[\mathbf{f}](\mathbf{x})+\mathcal{D}_{\partial \Omega}[\mathbf{g}](\mathbf{x}), \quad \mathbf{x} \in \Omega .
$$

Then there is a unique pair $\left(\boldsymbol{\varphi}_{1}, \boldsymbol{\varphi}_{2}\right) \in H_{\Psi}^{-1 / 2}\left(\partial D_{1}\right) \times H_{\Psi}^{-1 / 2}\left(\partial D_{2}\right)$ such that

$$
\mathbf{u}(\mathbf{x})=\mathbf{H}_{\Omega}(\mathbf{x})+\mathcal{S}_{\partial D_{1}}\left[\boldsymbol{\varphi}_{1}\right](\mathbf{x})+\mathcal{S}_{\partial D_{2}}\left[\boldsymbol{\varphi}_{2}\right](\mathbf{x}), \quad \mathbf{x} \in \Omega
$$

In fact, $\boldsymbol{\varphi}_{j}$ is given by $\boldsymbol{\varphi}_{j}=\left.\partial_{\nu} \mathbf{u}\right|_{+}$on $\partial D_{j}$ for $j=1,2$.

Proposition 2.2. Let $\mathbf{u}$ be the solution to 2.10). Then there is a unique pair $\left(\boldsymbol{\varphi}_{1}, \boldsymbol{\varphi}_{2}\right) \in$ $H_{\Psi}^{-1 / 2}\left(\partial D_{1}\right) \times H_{\Psi}^{-1 / 2}\left(\partial D_{2}\right)$ such that

$$
\mathbf{u}(\mathbf{x})=\mathbf{H}(\mathbf{x})+\mathcal{S}_{\partial D_{1}}\left[\boldsymbol{\varphi}_{1}\right](\mathbf{x})+\mathcal{S}_{\partial D_{2}}\left[\boldsymbol{\varphi}_{2}\right](\mathbf{x}), \quad \mathbf{x} \in D^{e} .
$$

In fact, $\boldsymbol{\varphi}_{j}$ is given by $\boldsymbol{\varphi}_{j}=\left.\partial_{\nu} \mathbf{u}\right|_{+}$on $\partial D_{j}$ for $j=1,2$.

Note that $\int_{\partial D_{j}} \boldsymbol{\varphi}_{j}=0$, which holds because $\boldsymbol{\varphi}_{j}$ belongs to $H_{\Psi}^{-1 / 2}\left(\partial D_{j}\right)$. So, we have $\mathcal{S}_{\partial D_{j}}\left[\boldsymbol{\varphi}_{j}\right](\mathbf{x})=O\left(|\mathbf{x}|^{-1}\right)$ as $|\mathbf{x}| \rightarrow \infty$. Thus $\mathbf{u}$ given by 2.19 satisfies the last condition in 2.10 .

Note that since the domains $D_{1}$ and $D_{2}$ are assumed to have $C^{4}$ boundaries, the solutions to 2.6 and 2.10 are $C^{3, \alpha}$ in $\Omega \backslash\left(D_{1} \cup D_{2}\right)$ including $\partial D_{1} \cup \partial D_{2}$ for any $0<\alpha<1$.

We now prove an analogue of the addition formula for $\boldsymbol{\Gamma}(\mathbf{x}-\mathbf{y})$. Let $\left\{\mathbf{e}_{1}, \mathbf{e}_{2}\right\}$ be the standard basis for $\mathbb{R}^{2}$. For $n \in \mathbb{Z}$ let

$$
P_{n}(\mathbf{x})=r^{|n|} e^{i n \theta}
$$

where $(r, \theta)$ denotes the polar coordinates of $\mathbf{x}$. Let

$$
\begin{aligned}
& \mathbf{v}_{n}^{(i)}(\mathbf{x})=\alpha_{1} P_{n}(\mathbf{x}) \mathbf{e}_{i}-\alpha_{2} x_{i} \nabla P_{n}(\mathbf{x}), \quad i=1,2, \\
& \mathbf{w}_{n}(\mathbf{x})=\alpha_{2} \nabla P_{n}(\mathbf{x}) .
\end{aligned}
$$

Since $P_{n}$ is harmonic in $\mathbb{R}^{2}$, one can easily see that $\mathbf{w}_{n}$ is a solution to the Lamé system in $\mathbb{R}^{2}$. To show that $\mathbf{v}_{n}^{(i)}$ is a solution to the Lamé system in $\mathbb{R}^{2}$, we prove a more general fact:

Lemma 2.3. If $h$ is a harmonic function, then a vector-valued function $\mathbf{v}$ of the form

$$
\mathbf{v}(\mathbf{x})=\alpha_{1} h(\mathbf{x}) \mathbf{e}_{j}-\alpha_{2} x_{j} \nabla h(\mathbf{x})
$$

for $j=1,2$, is a solution of the Lamé system, namely, $\mathcal{L}_{\lambda, \mu} \mathbf{v}=0$.

Proof. We only prove the case when $j=1$. Let us write $\mathbf{v}=\left(v_{1}, v_{2}\right)^{T}$. Simple computations show that

$$
\Delta v_{1}=-2 \alpha_{2} \partial_{1}^{2} h
$$

and

$$
\Delta v_{2}=-2 \alpha_{2} \partial_{1} \partial_{2} h
$$


We also have

$$
\nabla \cdot \mathbf{v}=\alpha_{1} \partial_{1} h-\alpha_{2}\left(x_{1} \Delta h+\partial_{1} h\right)=\left(\alpha_{1}-\alpha_{2}\right) \partial_{1} h .
$$

Therefore we obtain

$$
\begin{aligned}
{[\mu \Delta \mathbf{v}+(\lambda+\mu) \nabla(\nabla \cdot \mathbf{v})] \cdot \mathbf{e}_{k} } & =-2 \mu \alpha_{2} \partial_{1} \partial_{k} h+(\lambda+\mu)\left(\alpha_{1}-\alpha_{2}\right) \partial_{1} \partial_{k} h \\
& =\left(-\frac{1}{2 \pi} \frac{\lambda+\mu}{\lambda+2 \mu}+\frac{1}{2 \pi} \frac{\lambda+\mu}{\lambda+2 \mu}\right) \partial_{1} \partial_{k} h=0
\end{aligned}
$$

for $k=1,2$. This completes the proof.

We obtain the following proposition.

Proposition 2.4. The fundamental solution $\boldsymbol{\Gamma}$ admits the following series expansion: for $|\mathbf{x}|>|\mathbf{y}|$ and for any constant vector $\mathbf{b}$ in $\mathbb{R}^{2}$

$$
\begin{aligned}
\boldsymbol{\Gamma}(\mathbf{x}-\mathbf{y}) \mathbf{b}= & -\sum_{n \neq 0} \frac{1}{2|n|} \frac{e^{-i n \theta}}{r^{|n|}} \sum_{i=1}^{2}\left(\mathbf{v}_{n}^{(i)}(\mathbf{y}) \cdot \mathbf{b}\right) \mathbf{e}_{i} \\
& +\sum_{n \neq 0} \frac{1}{2|n|} \frac{\mathbf{x} e^{-i n \theta}}{r^{|n|}}\left(\mathbf{w}_{n}(\mathbf{y}) \cdot \mathbf{b}\right)+\alpha_{1} \ln |\mathbf{x}| \mathbf{b}
\end{aligned}
$$

where $\mathbf{x}=(r, \theta)$ in the polar coordinates. Moreover, the series converges absolutely and uniformly in $\mathbf{x}$ and $\mathbf{y}$ provided that there are numbers $r_{1}$ and $r_{2}$ such that $|\mathbf{y}| \leq r_{1}<r_{2} \leq$ $|\mathbf{x}|$.

Proof. By (2.13), we have

$$
\boldsymbol{\Gamma}(\mathbf{x}-\mathbf{y}) \mathbf{b}=\alpha_{1} \ln |\mathbf{x}-\mathbf{y}| \mathbf{b}-\alpha_{2}\left(\nabla_{\mathbf{y}}(\ln |\mathbf{x}-\mathbf{y}|) \cdot \mathbf{b}\right)(\mathbf{x}-\mathbf{y})(-1)
$$

for any constant vector $\mathbf{b}$. The addition formula for $\ln |\mathbf{x}-\mathbf{y}|$ reads

$$
\ln |\mathbf{x}-\mathbf{y}|=\ln |\mathbf{x}|-\sum_{n \neq 0} \frac{1}{2|n|} \frac{e^{-i n \theta}}{r^{|n|}} P_{n}(\mathbf{y}) .
$$

By substituting this formula to the one above, we obtain (2.24).

\subsection{The exterior problem and the variational principle}

In this subsection we consider the following exterior Dirichlet problem for the Lamé system:

$$
\begin{cases}\mathcal{L}_{\lambda, \mu} \mathbf{v}=0 & \text { in } D^{e}, \\ \mathbf{v}=\mathbf{g} & \text { on } \partial D^{e}=\partial D_{1} \cup \partial D_{2},\end{cases}
$$

for $\mathbf{g} \in H^{1 / 2}\left(\partial D^{e}\right)^{2}:=H^{1 / 2}\left(\partial D_{1}\right)^{2} \times H^{1 / 2}\left(\partial D_{2}\right)^{2}$. We seek a solution in the function space $\mathcal{A}^{*}$ defined as follows: Let $\mathcal{A}$ be the collection of all $\mathbf{v} \in H_{\text {loc }}^{1}\left(D^{e}\right)$ such that there exists a $2 \times 2$ symmetric matrix $B$ such that

$$
\mathbf{v}(\mathbf{x})=\sum_{j=1}^{2} \partial_{j} \boldsymbol{\Gamma}(\mathbf{x}) B \mathbf{e}_{j}+O\left(|\mathbf{x}|^{-2}\right) \quad \text { as }|\mathbf{x}| \rightarrow \infty
$$


where $\left\{\mathbf{e}_{1}, \mathbf{e}_{2}\right\}$ is the standard basis of $\mathbb{R}^{2}$. We emphasize that $\mathbf{v}(\mathbf{x})=O\left(|\mathbf{x}|^{-1}\right)$ as $|\mathbf{x}| \rightarrow \infty$. We then define

$$
\mathcal{A}^{*}:=\left\{\mathbf{u}=\mathbf{v}+\sum_{j=1}^{3} b_{j} \Psi_{j} \mid \mathbf{v} \in \mathcal{A}, \quad b_{j}: \text { constant }\right\} .
$$

A proof of the following theorem is given in Appendix.

Theorem 2.5. For any $\mathbf{g} \in H^{1 / 2}\left(\partial D^{e}\right)^{2}$, 2.25) admits a unique solution in $\mathcal{A}^{*}$.

This theorem in a different form is proved in [13] when $D^{e}$ is the compliment of a simply connected domain. Here, $\partial D^{e}$ has two components, namely, $\partial D^{e}=\partial D_{1} \cup \partial D_{2}$. Moreover, the proof of this paper is completely different from that of [13]. It is worth mentioning that the term $\sum_{j=1}^{3} b_{j} \Psi_{j}$ plays the role of the solution corresponding to the component of $\mathbf{g}$ spanned by $\Psi_{j}, j=1,2,3$.

The condition 2.26) is somewhat unfamiliar. To motivate it we prove the following lemma. This lemma will be used in the proof of Theorem 2.5.

Lemma 2.6. (i) If $\boldsymbol{\varphi}=\left(\boldsymbol{\varphi}_{1}, \boldsymbol{\varphi}_{2}\right) \in H^{-1 / 2}\left(\partial D_{1}\right)^{2} \times H^{-1 / 2}\left(\partial D_{2}\right)^{2}$ and satisfies

$$
\int_{\partial D_{1}} \varphi_{1} \cdot \Psi_{k}+\int_{\partial D_{2}} \varphi_{2} \cdot \Psi_{k}=0, \quad k=1,2,3
$$

then $\mathbf{v}$, defined by

$$
\mathbf{v}(\mathbf{x})=\mathcal{S}_{\partial D_{1}}\left[\boldsymbol{\varphi}_{1}\right](\mathbf{x})+\mathcal{S}_{\partial D_{2}}\left[\boldsymbol{\varphi}_{2}\right](\mathbf{x}), \quad \mathbf{x} \in D^{e},
$$

belongs to $\mathcal{A}$.

(ii) If $\boldsymbol{\psi}=\left(\boldsymbol{\psi}_{1}, \boldsymbol{\psi}_{2}\right)$ belongs to $H^{1 / 2}\left(\partial D_{1}\right)^{2} \times H^{1 / 2}\left(\partial D_{2}\right)^{2}$, then $\mathbf{w}$, defined by

$$
\mathbf{w}(\mathbf{x})=\mathcal{D}_{\partial D_{1}}\left[\boldsymbol{\psi}_{1}\right](\mathbf{x})+\mathcal{D}_{\partial D_{2}}\left[\boldsymbol{\psi}_{2}\right](\mathbf{x}), \quad \mathbf{x} \in D^{e},
$$

belongs to $\mathcal{A}$.

Proof. If $\mathbf{y} \in \partial D^{e}$ and $|\mathbf{x}| \rightarrow \infty$, then by the Taylor expansion we have

$$
\boldsymbol{\Gamma}(\mathbf{x}-\mathbf{y})=\boldsymbol{\Gamma}(\mathbf{x})+\sum_{j=1}^{2} \partial_{j} \boldsymbol{\Gamma}(\mathbf{x}) y_{j}+O\left(|\mathbf{x}|^{-2}\right) .
$$

So $\mathbf{v}$ defined by 2.29 takes the form

$$
\mathbf{v}(\mathbf{x})=\boldsymbol{\Gamma}(\mathbf{x}) \int_{\partial D^{e}} \boldsymbol{\varphi}+\sum_{j=1}^{2} \partial_{j} \boldsymbol{\Gamma}(\mathbf{x}) \int_{\partial D^{e}} y_{j} \boldsymbol{\varphi}+O\left(|\mathbf{x}|^{-2}\right) .
$$

Here and throughout this paper we use $\int_{\partial D^{e}} \boldsymbol{\varphi}$ to denote $\int_{\partial D_{1}} \boldsymbol{\varphi}_{1}+\int_{\partial D_{2}} \boldsymbol{\varphi}_{2}$ for ease of notation. The assumption (2.28) for $k=1,2$ implies that the first term in the right-hand side of 2.32 vanishes. Define the matrix $B:=\left(b_{i j}\right)_{i, j=1,2}$ by

$$
\left[\begin{array}{l}
b_{11} \\
b_{21}
\end{array}\right]:=\int_{\partial D^{e}} y_{1} \varphi \quad \text { and } \quad\left[\begin{array}{l}
b_{12} \\
b_{22}
\end{array}\right]:=\int_{\partial D^{e}} y_{2} \varphi
$$


Then, we may rewrite 2.32 as

$$
\mathbf{v}(\mathbf{x})=\sum_{j=1}^{2} \partial_{j} \boldsymbol{\Gamma}(\mathbf{x}) B \mathbf{e}_{j}+O\left(|\mathbf{x}|^{-2}\right) \quad \text { as }|\mathbf{x}| \rightarrow \infty .
$$

Note that the assumption 2.28 for $k=3$ implies $b_{12}=b_{21}$, namely, $B$ is symmetric.

To prove (ii), let $\mathbf{u}_{j}$ be the solution to $\mathcal{L}_{\lambda, \mu} \mathbf{u}_{j}=0$ in $D_{j}$ and $\mathbf{u}_{j}=\boldsymbol{\psi}_{j}$ on $\partial D_{j}$. Then $\partial_{\nu} \mathbf{u}_{j} \in H_{\Psi}^{-1 / 2}\left(\partial D_{j}\right)$ and Green's formula for the Lamé system shows that the following holds:

$$
\mathcal{D}_{\partial D_{1}}\left[\boldsymbol{\psi}_{1}\right](\mathbf{x})=\mathcal{S}_{\partial D_{1}}\left[\partial_{\nu} \mathbf{u}_{1}\right](\mathbf{x}), \quad \mathcal{D}_{\partial D_{2}}\left[\boldsymbol{\psi}_{2}\right](\mathbf{x})=\mathcal{S}_{\partial D_{2}}\left[\partial_{\nu} \mathbf{u}_{2}\right](\mathbf{x}), \quad \mathbf{x} \in D^{e}
$$

So, we have

$$
\mathbf{w}(\mathbf{x})=\mathcal{S}_{\partial D_{1}}\left[\partial_{\nu} \mathbf{u}_{1}\right](\mathbf{x})+\mathcal{S}_{\partial D_{2}}\left[\partial_{\nu} \mathbf{u}_{2}\right](\mathbf{x}), \quad \mathbf{x} \in D^{e} .
$$

So, (ii) follows from (i).

The most important property of the function of the form $\sum_{j=1}^{2} \partial_{j} \boldsymbol{\Gamma}(\mathbf{x}) B \mathbf{e}_{j}$ lies in the following fact.

Lemma 2.7. Let $\mathbf{v}(\mathbf{x})=\sum_{j=1}^{2} \partial_{j} \boldsymbol{\Gamma}(\mathbf{x})$ Be $\mathbf{e}_{j}$ for some symmetric matrix $B$. Then the following holds for any simple closed Lipschitz curve $C$ such that $0 \notin C$ :

$$
\int_{C} \partial_{\nu} \mathbf{v} \cdot \Psi_{k}=0, \quad k=1,2,3
$$

Proof. Since the cases of $k=1,2$ are easier to prove, we only consider the case of $k=3$. Let $U$ be the bounded domain enclosed by $C$. If $0 \notin U$, then by Green's formula for the Lamé system, we have

$$
\int_{C} \partial_{\nu} \mathbf{v} \cdot \Psi_{3}=\int_{U} \mathbb{C} \widehat{\nabla} \mathbf{v}: \widehat{\nabla} \Psi_{3}=0 .
$$

Suppose that $0 \in U$. Then choose $B_{r}$, the disk of radius $r$ centered at 0 , so that $\overline{B_{r}} \subset U$. Then, we see that

$$
\int_{C} \partial_{\nu} \mathbf{v} \cdot \Psi_{3}=\int_{\partial B_{r}} \partial_{\nu} \mathbf{v} \cdot \Psi_{3}
$$

Straightforward but tedious computations show that on $\partial B_{r}$

$$
\partial_{\nu} \mathbf{v} \cdot \Psi_{3}=\frac{1}{2 \pi r}\left(b_{21}-b_{12}\right)+\frac{\lambda+\mu}{\lambda+2 \mu}\left(b_{11}-\frac{b_{12}}{2}-\frac{b_{21}}{2}-b_{22}\right) \frac{\sin 2 \theta}{2 \pi r},
$$

where $(r, \theta)$ is the polar coordinates. So we obtain

$$
\int_{\partial B_{R}} \partial_{\nu} \mathbf{v} \cdot \Psi_{3}=b_{21}-b_{12}
$$

Since $b_{12}=b_{21}, 2.33$ follows.

The following lemma shows that Green's formula holds for $\mathbf{u}, \mathbf{v} \in \mathcal{A}^{*}$. It is worth mentioning that the - sign appears on the right-hand side of (2.34) below since the normal vector on $\partial D^{e}$ is directed outward. 
Lemma 2.8. If $\mathbf{u}, \mathbf{v} \in \mathcal{A}^{*}$ and $\mathcal{L}_{\lambda, \mu} \mathbf{u}=0$ in $D^{e}$, then

$$
\int_{D^{e}} \mathbb{C} \widehat{\nabla} \mathbf{u}: \widehat{\nabla} \mathbf{v}=-\left.\int_{\partial D^{e}} \partial_{\nu} \mathbf{u}\right|_{+} \cdot \mathbf{v}
$$

where the left-hand side is understood to be

$$
\int_{D^{e}} \mathbb{C} \widehat{\nabla} \mathbf{u}: \hat{\nabla} \mathbf{v}=\lim _{R \rightarrow \infty} \int_{B_{R} \backslash\left(D_{1} \cup D_{2}\right)} \mathbb{C} \widehat{\nabla} \mathbf{u}: \widehat{\nabla} \mathbf{v}
$$

Proof. We have

$$
\int_{B_{R} \backslash\left(D_{1} \cup D_{2}\right)} \mathbb{C} \hat{\nabla} \mathbf{u}: \hat{\nabla} \mathbf{v}=-\left.\int_{\partial D^{e}} \partial_{\nu} \mathbf{u}\right|_{+} \cdot \mathbf{v}+\left.\int_{\partial B_{R}} \partial_{\nu} \mathbf{u}\right|_{+} \cdot \mathbf{v} .
$$

So, it suffices to prove that

$$
\left.\lim _{R \rightarrow \infty} \int_{\partial B_{R}} \partial_{\nu} \mathbf{u}\right|_{+} \cdot \mathbf{v}=0
$$

Let $\mathbf{u}=\mathbf{u}_{1}+\mathbf{u}_{2}+\mathbf{u}_{3}$ where $\mathbf{u}_{1}$ is of the form $\sum_{j=1}^{2} \partial_{j} \boldsymbol{\Gamma}(\mathbf{x}) B \mathbf{e}_{j}, \mathbf{u}_{2}(\mathbf{x})=O\left(|\mathbf{x}|^{-2}\right)$, and $\mathbf{u}_{3}$ is of the form $\sum_{k=1}^{3} a_{k} \Psi_{k}$. We also let $\mathbf{v}=\mathbf{v}_{1}+\mathbf{v}_{2}$ where $\mathbf{v}_{1}(\mathbf{x})=O\left(|\mathbf{x}|^{-1}\right)$ and $\mathbf{v}_{2}$ is of the form $\sum_{k=1}^{3} b_{k} \Psi_{k}$. Since $\partial_{\nu} \mathbf{u}_{3}=0$ on $\partial B_{R}$ for any $R$, we have

$$
\left.\int_{\partial B_{R}} \partial_{\nu} \mathbf{u}\right|_{+} \cdot \mathbf{v}=\left.\int_{\partial B_{R}} \partial_{\nu}\left(\mathbf{u}_{1}+\mathbf{u}_{2}\right)\right|_{+} \cdot\left(\mathbf{v}_{1}+\mathbf{v}_{2}\right) .
$$

We see

$$
\lim _{R \rightarrow \infty}\left[\left.\int_{\partial B_{R}} \partial_{\nu}\left(\mathbf{u}_{1}+\mathbf{u}_{2}\right)\right|_{+} \cdot \mathbf{v}_{1}+\left.\int_{\partial B_{R}} \partial_{\nu} \mathbf{u}_{2}\right|_{+} \cdot \mathbf{v}_{2}\right]=0
$$

by considering the decay at $\infty$ of the functions involved. We also have from 2.33

$$
\left.\int_{\partial B_{R}} \partial_{\nu} \mathbf{u}_{1}\right|_{+} \cdot \mathbf{v}_{2}=0
$$

This completes the proof.

The following variational principle for the exterior Dirichlet problem plays a crucial role in what follows.

Lemma 2.9. Define

$$
\mathcal{E}_{D^{e}}[\mathbf{w}]:=\int_{D^{e}} \mathbb{C} \widehat{\nabla} \mathbf{w}: \widehat{\nabla} \mathbf{w}
$$

Let $\mathbf{u}$ be the solution in $\mathcal{A}^{*}$ to 2.25) with $\mathbf{g} \in H^{1 / 2}\left(\partial D^{e}\right)^{2}$. Then the following variational principle holds:

$$
\mathcal{E}_{D^{e}}[\mathbf{u}]=\min _{\mathbf{w} \in W_{\mathbf{g}}} \mathcal{E}_{D^{e}}[\mathbf{w}]
$$

where

$$
W_{\mathbf{g}}=\left\{\mathbf{w} \in \mathcal{A}^{*}:\left.\mathbf{w}\right|_{\partial D^{e}}=\mathbf{g}\right\}
$$


Proof. Let $\mathbf{w} \in W_{\mathbf{g}}$. By Lemma 2.8, we have

$$
\int_{D^{e}} \mathbb{C} \widehat{\nabla} \mathbf{u}: \widehat{\nabla} \mathbf{u}=-\left.\int_{\partial D^{e}} \partial_{\nu} \mathbf{u}\right|_{+} \cdot \mathbf{g}=\int_{D^{e}} \mathbb{C} \widehat{\nabla} \mathbf{u}: \widehat{\nabla} \mathbf{w}
$$

By the Cauchy-Schwartz inequality, we have

$$
\int_{D^{e}} \mathbb{C} \widehat{\nabla} \mathbf{u}: \widehat{\nabla} \mathbf{u}=\int_{D^{e}} \mathbb{C} \widehat{\nabla} \mathbf{u}: \widehat{\nabla} \mathbf{w} \leq \frac{1}{2}\left(\int_{D^{e}} \mathbb{C} \widehat{\nabla} \mathbf{u}: \widehat{\nabla} \mathbf{u}+\int_{D^{e}} \mathbb{C} \widehat{\nabla} \mathbf{w}: \widehat{\nabla} \mathbf{w}\right) .
$$

Thus (2.37) holds.

\subsection{An estimate for the free space problem}

The purpose of this subsection is to prove the following proposition which will be used in section 5 .

Proposition 2.10. Let $\mathbf{u}$ be the solution to (2.10) for a given $\mathbf{H}$. Then for any disk $B$ centered at 0 containing $\overline{D_{1} \cup D_{2}}$ and for $k=0,1,2, \ldots$, there is a constant $C_{k}$ independent of $\epsilon$ (and $\mathbf{H})$ such that

$$
\left\|\nabla^{k}(\mathbf{u}-\mathbf{H})\right\|_{L^{\infty}\left(\mathbb{R}^{2} \backslash B\right)} \leq C_{k}\|\mathbf{H}\|_{H^{1}(B)} .
$$

The main emphasis of (2.38) is that the estimate holds independently of $\epsilon$, the distance between $D_{1}$ and $D_{2}$. It shows that even if $\mathbf{u}$ depends on $\epsilon$, the dependence is negligible far away from the inclusions.

To prove Proposition 2.10, we begin with the following lemma.

Lemma 2.11. Let $\mathbf{u}$ be the solution to (2.10). There is a constant $C$ independent of $\epsilon$ and $\mathbf{H}$ such that

$$
\mathcal{E}_{D^{e}}[\mathbf{u}-\mathbf{H}] \leq C\|\mathbf{H}\|_{H^{1}(B)}^{2},
$$

where $\mathcal{E}_{D^{e}}$ is defined in $(2.36)$ and $B$ is a disk centered at 0 containing $\overline{D_{1} \cup D_{2}}$.

Proof. We first observe from (2.7) and the second condition in 2.10 that

$$
\left.\int_{\partial D_{i}} \partial_{\nu} \mathbf{u}\right|_{+} \cdot \mathbf{u}=0, \quad i=1,2
$$

Since $\mathcal{L}_{\lambda, \mu} \mathbf{H}=0$ in $\mathbb{R}^{2}$, we also have

$$
\left.\int_{\partial D_{i}} \partial_{\nu} \mathbf{H}\right|_{+} \cdot \mathbf{u}=0, \quad i=1,2
$$

So we have

$$
\begin{aligned}
\mathcal{E}_{D^{e}}[\mathbf{u}-\mathbf{H}] & =\int_{D^{e}} \mathbb{C} \widehat{\nabla}(\mathbf{u}-\mathbf{H}): \widehat{\nabla}(\mathbf{u}-\mathbf{H}) \\
& =-\left.\int_{\partial D^{e}} \partial_{\nu}(\mathbf{u}-\mathbf{H})\right|_{+} \cdot(\mathbf{u}-\mathbf{H})=\left.\int_{\partial D^{e}} \partial_{\nu}(\mathbf{u}-\mathbf{H})\right|_{+} \cdot \mathbf{H} .
\end{aligned}
$$


Let $R$ be the radius of $B$ and let $r$ be such that $r<R$ and $\overline{D_{1} \cup D_{2}} \subset B_{r}$. Let $\chi$ be a smooth radial function such that $\chi(\mathbf{x})=1$ if $|\mathbf{x}| \leq r$ and $\chi(\mathbf{x})=0$ if $|\mathbf{x}| \geq R$. Let $\mathbf{w}:=-\chi \mathbf{H}$. Then we have

$$
\int_{D^{e}} \mathbb{C} \widehat{\nabla}(\mathbf{u}-\mathbf{H}): \hat{\nabla} \mathbf{w}=-\left.\int_{\partial D^{e}} \partial_{\nu}(\mathbf{u}-\mathbf{H})\right|_{+} \cdot \mathbf{w}=\left.\int_{\partial D^{e}} \partial_{\nu}(\mathbf{u}-\mathbf{H})\right|_{+} \cdot \mathbf{H} .
$$

It then follows that

$$
\mathcal{E}_{D^{e}}[\mathbf{u}-\mathbf{H}]=\int_{D^{e}} \mathbb{C} \widehat{\nabla}(\mathbf{u}-\mathbf{H}): \widehat{\nabla} \mathbf{w} \leq \frac{1}{2}\left(\mathcal{E}_{D^{e}}[\mathbf{u}-\mathbf{H}]+\mathcal{E}_{D^{e}}[\mathbf{w}]\right) .
$$

So we have

$$
\mathcal{E}_{D^{e}}[\mathbf{u}-\mathbf{H}] \leq \mathcal{E}_{D^{e}}[\mathbf{w}] \leq C\|\mathbf{H}\|_{H^{1}(B)}^{2} .
$$

The proof is completed.

Proof of Proposition 2.10. By Proposition 2.2, the solution $\mathbf{u}$ is represented as

$$
\mathbf{u}=\mathbf{H}+\mathcal{S}_{\partial D_{1}}\left[\boldsymbol{\varphi}_{1}\right]+\mathcal{S}_{\partial D_{2}}\left[\boldsymbol{\varphi}_{2}\right]
$$

with $\boldsymbol{\varphi}_{j}=\left.\partial_{\nu} \mathbf{u}\right|_{+}$on $\partial D_{j}, j=1,2$. Proposition 2.4 yields

$$
(\mathbf{u}-\mathbf{H})(\mathbf{x})=\sum_{n \neq 0} \frac{1}{2|n|} \frac{e^{-i n \theta}}{r^{|n|}}\left(-M_{n}^{(1)} \mathbf{e}_{1}-M_{n}^{(2)} \mathbf{e}_{2}+M_{n}^{(3)} \mathbf{x}\right), \quad r=|\mathbf{x}| \geq R,
$$

where $R$ is the radius of $B$ and $M_{n}^{(i)}$ is given by

$$
\begin{aligned}
& M_{n}^{(i)}=\int_{\partial D_{1}} \mathbf{v}_{n}^{(i)} \cdot \boldsymbol{\varphi}_{1} d \sigma+\int_{\partial D_{2}} \mathbf{v}_{n}^{(i)} \cdot \boldsymbol{\varphi}_{2} d \sigma, \quad i=1,2, \\
& M_{n}^{(3)}=\int_{\partial D_{1}} \mathbf{w}_{n} \cdot \boldsymbol{\varphi}_{1} d \sigma+\int_{\partial D_{2}} \mathbf{w}_{n} \cdot \boldsymbol{\varphi}_{2} d \sigma .
\end{aligned}
$$

Observe that the dependence of $\mathbf{u}-\mathbf{H}$ on $\epsilon$ is contained only in the coefficients $M_{n}^{(i)}$, $i=1,2,3$.

Let $r$ be such that $r<R$ and $B_{r}$ contains $\overline{D_{1} \cup D_{2}}$. We now show that there is a constant $C$ independent of $\epsilon$ and $n$ such that

$$
\left|M_{n}^{(i)}\right| \leq C|n| r^{|n|+2}\|\mathbf{H}\|_{H^{1}\left(B_{r}\right)}
$$

for all $n \neq 0$ and $i=1,2,3$.

For simplicity, we consider only $i=1$. The other cases can be proved in the exactly same way. Let $\mathbf{v}$ be the solution to 2.10 when $\mathbf{H}=\mathbf{v}_{n}^{(1)}$. Since $\boldsymbol{\varphi}_{j}=\left.\partial_{\nu} \mathbf{u}\right|_{+}$on $\partial D_{j}$ and $\left.\mathbf{v}\right|_{\partial D_{j}} \in \Psi$, we have using 2.7 that

$$
\begin{aligned}
M_{n}^{(1)} & =\left.\int_{\partial D^{e}} \mathbf{v}_{n}^{(1)} \cdot \partial_{\nu} \mathbf{u}\right|_{+} \\
& =-\left.\int_{\partial D^{e}}\left(\mathbf{v}-\mathbf{v}_{n}^{(1)}\right) \cdot \partial_{\nu} \mathbf{u}\right|_{+} \\
& =-\left.\int_{\partial D^{e}}\left(\mathbf{v}-\mathbf{v}_{n}^{(1)}\right) \cdot \partial_{\nu}(\mathbf{u}-\mathbf{H})\right|_{+}+\left.\int_{\partial D^{e}} \mathbf{v}_{n}^{(1)} \cdot \partial_{\nu} \mathbf{H}\right|_{+} \\
& =\int_{D^{e}} \mathbb{C} \widehat{\nabla}\left(\mathbf{v}-\mathbf{v}_{n}^{(1)}\right): \widehat{\nabla}(\mathbf{u}-\mathbf{H})+\left.\int_{\partial D^{e}} \mathbf{v}_{n}^{(1)} \cdot \partial_{\nu} \mathbf{H}\right|_{+} .
\end{aligned}
$$


So, by applying Lemma 2.11 to $\mathcal{E}_{D^{e}}\left[\mathbf{v}-\mathbf{v}_{n}^{(1)}\right]$ and $\mathcal{E}_{D^{e}}[\mathbf{u}-\mathbf{H}]$ on $B_{r}$, we obtain

$$
\begin{aligned}
\left|M_{n}^{(1)}\right| & \leq \mathcal{E}_{D^{e}}\left[\mathbf{v}-\mathbf{v}_{n}^{(1)}\right]^{1 / 2} \mathcal{E}_{D^{e}}[\mathbf{u}-\mathbf{H}]^{1 / 2}+\sum_{i=1}^{2}\left\|\mathbf{v}_{n}^{(1)}\right\|_{H^{1 / 2}\left(\partial D_{i}\right)}\left\|\partial_{\nu} \mathbf{H}\right\|_{H^{-1 / 2}\left(\partial D_{i}\right)} \\
& \leq C\left\|\mathbf{v}_{n}^{(1)}\right\|_{H^{1}\left(B_{r}\right)}\|\mathbf{H}\|_{H^{1}\left(B_{r}\right)}+\sum_{i=1}^{2}\left\|\mathbf{v}_{n}^{(1)}\right\|_{H^{1}\left(D_{i}\right)}\|\mathbf{H}\|_{H^{1}\left(D_{i}\right)} \\
& \leq C\left\|\mathbf{v}_{n}^{(1)}\right\|_{H^{1}\left(B_{r}\right)}\|\mathbf{H}\|_{H^{1}\left(B_{r}\right)} .
\end{aligned}
$$

Here and throughout this paper, the constant $C$ appearing in the course of estimations may differ at each occurrence. Since $\mathbf{v}_{n}^{(1)}$ is a homogeneous polynomial of order $n$, there is a constant $C$ independent of $n$ such that

$$
\left\|\mathbf{v}_{n}^{(1)}\right\|_{H^{1}\left(B_{r}\right)} \leq C|n| r^{|n|+2},
$$

assuming that $r>1$.

It follows from (2.41) and $(2.44)$ that

$$
\begin{aligned}
\|\mathbf{u}-\mathbf{H}\|_{L^{\infty}\left(\mathbb{R}^{2} \backslash B_{R}\right)} & \leq C \sum_{n \neq 0} \frac{1}{2|n|} \frac{1}{R^{|n|}}\left(|n| r^{|n|+2}\right)\|\mathbf{H}\|_{H^{1}\left(B_{r}\right)} \\
& \leq C \sum_{n \neq 0}\left(\frac{r}{R}\right)^{|n|}\|\mathbf{H}\|_{H^{1}(B)} \leq C\|\mathbf{H}\|_{H^{1}(B)} .
\end{aligned}
$$

This proves 2.38 for $k=0$.

If $k>0$, we differentiate (2.41) to obtain (2.38). This completes the proof.

\subsection{Geometry of two inclusions}

In this subsection, we describe geometry of two inclusions $D_{1}$ and $D_{2}$. See Figure 2.1.

Suppose that there are unique points $\mathbf{z}_{1} \in \partial D_{1}$ and $\mathbf{z}_{2} \in \partial D_{2}$ such that

$$
\left|\mathbf{z}_{1}-\mathbf{z}_{2}\right|=\operatorname{dist}\left(D_{1}, D_{2}\right) .
$$

We assume that $D_{j}$ is strictly convex near $\mathbf{z}_{j}$, namely, there is a common neighborhood $U$ of $\mathbf{z}_{1}$ and $\mathbf{z}_{2}$ such that $D_{j} \cap U$ is strictly convex for $j=1,2$. Moreover, we assume that

$$
\operatorname{dist}\left(D_{1}, D_{2} \backslash U\right) \geq C \text { and } \operatorname{dist}\left(D_{2}, D_{1} \backslash U\right) \geq C
$$

for some positive constant $C$ independent of $\epsilon$. This assumption says that other than neighborhoods of $\mathbf{z}_{1}$ and $\mathbf{z}_{2}, D_{1}$ and $D_{2}$ are at some distance to each other. We need one more assumption: the center of the circle which is osculating $D_{j}$ at $\mathbf{z}_{j}$ lies inside $D_{j}$ $(j=1,2)$. This assumption is needed for defining the singular function $\mathbf{q}_{3}$ in (3.15) later. We emphasize that strictly convex domains satisfy all the assumptions.

Let $\kappa_{j}$ be the curvature of $\partial D_{j}$ at $\mathbf{z}_{j}$. Let $B_{j}$ be the disk osculating to $D_{j}$ at $\mathbf{z}_{j}$ $(j=1,2)$. Then the radius $r_{j}$ of $B_{j}$ is given by $r_{j}=1 / \kappa_{j}$. Let $R_{j}$ be the reflection with respect to $\partial B_{j}$ and let $\mathbf{p}_{1}$ and $\mathbf{p}_{2}$ be the unique fixed points of the combined reflections $R_{1} \circ R_{2}$ and $R_{2} \circ R_{1}$, respectively. 


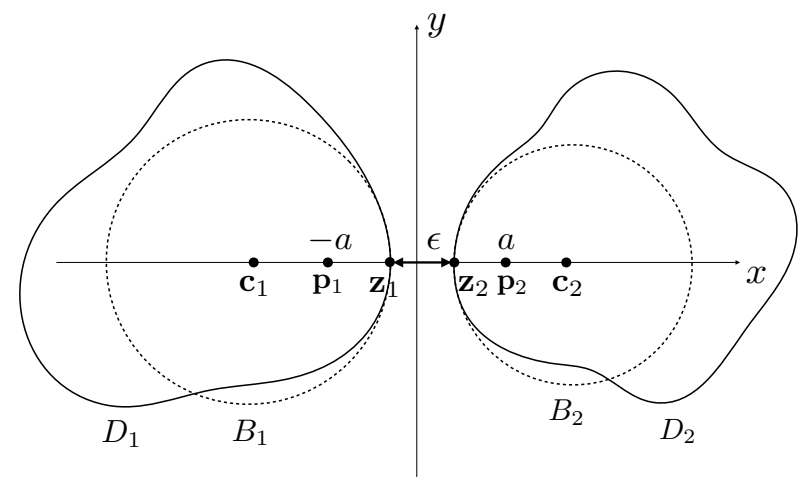

Figure 2.1: Geometry of the two inclusions and osculating circles

Let $\mathbf{n}$ be the unit vector in the direction of $\mathbf{p}_{2}-\mathbf{p}_{1}$ and let $\mathbf{t}$ be the unit vector perpendicular to $\mathbf{n}$ such that $(\mathbf{n}, \mathbf{t})$ is positively oriented. We set $(x, y) \in \mathbb{R}^{2}$ to be the Cartesian coordinates such that $\mathbf{p}=\left(\mathbf{p}_{1}+\mathbf{p}_{2}\right) / 2$ is the origin and the $x$-axis is parallel to $\mathbf{n}$. Then one can see (see [5]) that $\mathbf{p}_{1}$ and $\mathbf{p}_{2}$ are written as

$$
\mathbf{p}_{1}=(-a, 0) \quad \text { and } \quad \mathbf{p}_{2}=(a, 0),
$$

where the constant $a$ is given by

$$
a:=\frac{\sqrt{\epsilon} \sqrt{\left(2 r_{1}+\epsilon\right)\left(2 r_{2}+\epsilon\right)\left(2 r_{1}+2 r_{2}+\epsilon\right)}}{2\left(r_{1}+r_{2}+\epsilon\right)},
$$

from which one can infer

$$
a=\sqrt{\frac{2}{\kappa_{1}+\kappa_{2}}} \sqrt{\epsilon}+O\left(\epsilon^{3 / 2}\right) .
$$

Then the center $\mathbf{c}_{i}$ of $B_{i}(i=1,2)$ is given by

$$
\mathbf{c}_{i}=\left((-1)^{i} \sqrt{r_{i}^{2}+a^{2}}, 0\right)=\left((-1)^{i} r_{i}+O(\epsilon), 0\right) .
$$

So we have

$$
\mathbf{z}_{i}=(-1)^{i+1}\left(r_{i}-\sqrt{r_{i}^{2}+a^{2}}, 0\right)=\left((-1)^{i} \frac{\kappa_{i}}{\kappa_{1}+\kappa_{2}} \epsilon+O\left(\epsilon^{2}\right), 0\right) .
$$

Let us consider the narrow region between $D_{1}$ and $D_{2}$. See Figure 2.2, There exists $L>0$ (independent of $\epsilon$ ) and functions $f_{1}, f_{2}:[-L, L] \rightarrow \mathbb{R}$ such that

$$
\mathbf{z}_{1}=\left(-f_{1}(0), 0\right), \quad \mathbf{z}_{2}=\left(f_{2}(0), 0\right), \quad f_{1}^{\prime}(0)=f_{2}^{\prime}(0)=0,
$$

and $\partial D_{1}$ and $\partial D_{2}$ are graphs of $-f_{1}(y)$ and $f_{2}(y)$ for $|y|<L$, i.e.,

$$
\mathbf{x}_{1}(y):=\left(-f_{1}(y), y\right) \in \partial D_{1} \quad \text { and } \quad \mathbf{x}_{2}(y):=\left(f_{2}(y), y\right) \in \partial D_{2} .
$$




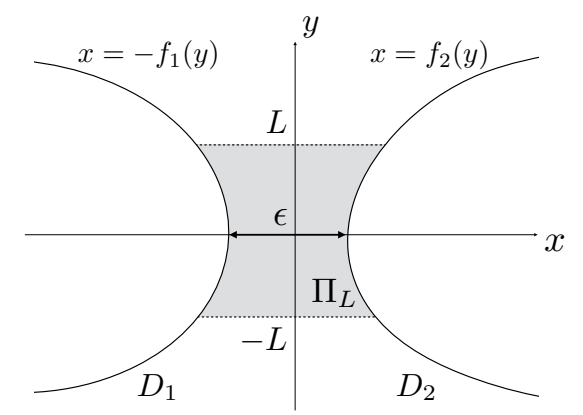

Figure 2.2: Geometry of the narrow gap region $\Pi_{L}$

Since $D_{i}$ is strictly convex near $\mathbf{z}_{j}, f_{1}$ is strictly convex. Note that, for $i=1,2$ and $|y|<L$,

$$
f_{i}(y)=\frac{\kappa_{i}}{\kappa_{1}+\kappa_{2}} \epsilon+\frac{1}{2 !} \kappa_{i} y^{2}+\frac{1}{3 !} \omega_{i} y^{3}+O\left(\epsilon^{2}+y^{4}\right)
$$

for some constant $\omega_{i}$. Let us define for later use a constant $\tau$ as

$$
\tau=\left|\kappa_{1}-\kappa_{2}\right|+\left|\omega_{1}\right|+\left|\omega_{2}\right| .
$$

We denote by $\Pi_{l}$ for $0<l \leq L$ the narrow region between $D_{1}$ and $D_{2}$ defined as

$$
\Pi_{l}=\left\{(x, y) \in \mathbb{R}^{2}\left|-f_{1}(y)<x<f_{2}(y),\right| y \mid<l\right\} .
$$

\section{Singular functions and their properties}

Let $\Psi_{j}, j=1,2,3$, be the rigid motions defined in 2.5 and let $\mathbf{h}_{j}$ be the solution to the following problem:

$$
\begin{cases}\mathcal{L}_{\lambda, \mu} \mathbf{h}_{j}=0 & \text { in } D^{e}, \\ \mathbf{h}_{j}=-\frac{1}{2} \Psi_{j}(\mathbf{x}) & \text { on } \partial D_{1}, \\ \mathbf{h}_{j}=\frac{1}{2} \Psi_{j}(\mathbf{x}) & \text { on } \partial D_{2} .\end{cases}
$$

It turns out that $\mathbf{h}_{j}(j=1,2,3)$ captures the singular behavior of the solution $\mathbf{u}$ to (2.10). In fact, $\mathbf{u}$ can be decomposed in the following form:

$$
\mathbf{u}=\sum_{j=1}^{3} c_{j} \mathbf{h}_{j}+\mathbf{b}
$$

for some constants $c_{j}$, where $\nabla \mathbf{b}$ is bounded in a bounded domain containing the narrow region $\Pi_{L}$ between $D_{1}$ and $D_{2}$. In other words, the blow-up behavior of $\nabla \mathbf{u}$ is completely characterized by that of $\sum_{j=1}^{3} c_{j} \nabla \mathbf{h}_{j}$. We emphasize that $\left|\mathbf{h}_{j}\right|_{\partial D_{1}}-\left.\mathbf{h}_{j}\right|_{\partial D_{2}} \mid=1$ for $j=1,2$. So one expect that $\left|\nabla \mathbf{h}_{j}\right| \approx \epsilon^{-1}$ in the narrow region between $D_{1}$ and $D_{2}$. The function $\mathbf{h}_{3}$ has a weaker singularity since $\left|\mathbf{h}_{3}\right|_{\partial D_{1}}-\left.\mathbf{h}_{3}\right|_{\partial D_{2}}|=| \mathbf{x} \mid$.

The purpose of this section is to construct explicit singular functions, denoted by $\mathbf{q}_{j}$, which yield good approximations of $\mathbf{h}_{j}$ and to derive their important properties. 


\subsection{Construction of singular functions}

We begin with a brief review of the singular function for the electro-static case. Let $\mathbf{p}_{j}$ $(j=1,2)$ be the fixed points of the combined reflections given in 2.46$)$ and let

$$
q_{B}(\mathbf{x})=\frac{1}{2 \pi}\left(\ln \left|\mathbf{x}-\mathbf{p}_{1}\right|-\ln \left|\mathbf{x}-\mathbf{p}_{2}\right|\right) .
$$

This function was introduced in [35] and used in an essential way for characterization of the gradient blow-up in the context of electro-statics [1]. The most important property of $q_{B}(\mathbf{x})$ is that it takes constant values on $\partial B_{j}$, the circles osculating to $\partial D_{j}$ at $\mathbf{z}_{j}, j=1,2$. It is because $\partial B_{1}$ and $\partial B_{2}$ are circles of Apollonius of $\mathbf{p}_{1}$ and $\mathbf{p}_{2}$.

Note that $\frac{1}{2 \pi} \ln |\mathbf{x}|$ is a fundamental solution of the Laplacian and represents a point source of the electric field. So it is natural to expect that, even in the linear elasticity case, the point source functions may also characterize the gradient blow-up. There are various types of point source functions in linear elasticity which are often called nuclei of strain. We will use the following nuclei of strain as basic building blocks of the singular functions:

$$
\boldsymbol{\Gamma}(\mathbf{x}) \mathbf{e}_{1}, \quad \boldsymbol{\Gamma}(\mathbf{x}) \mathbf{e}_{2}, \quad \frac{\mathbf{x}}{|\mathbf{x}|^{2}}, \quad \frac{\mathbf{x}^{\perp}}{|\mathbf{x}|^{2}} .
$$

where $\mathbf{x}^{\perp}=(-y, x)$ for $\mathbf{x}=(x, y) \in \mathbb{R}^{2}$. These nuclei of strain have physical meanings: the function $\boldsymbol{\Gamma}(\mathbf{x}) \mathbf{e}_{j}$ represents the point force applied at the origin in the direction of $\mathbf{e}_{j}$, and the functions $\mathbf{x} /|\mathbf{x}|$ and $\mathbf{x}^{\perp} /|\mathbf{x}|$ represent the point source of the pressure and that of the moment located at the origin, respectively (see, for example, [33]).

We emphasize that the functions given in (3.4) are solutions to the Lamé system for $\mathbf{x} \neq 0$. In fact, the first two are solutions since they are columns of the fundamental solution, and so are the last two because of the following relations:

$$
\left\{\begin{array}{l}
\left(\alpha_{1}-\alpha_{2}\right) \frac{\mathbf{x}}{|\mathbf{x}|^{2}}=\partial_{1}\left(\boldsymbol{\Gamma}(\mathbf{x}) \mathbf{e}_{1}\right)+\partial_{2}\left(\boldsymbol{\Gamma}(\mathbf{x}) \mathbf{e}_{2}\right) \\
\left(\alpha_{1}+\alpha_{2}\right) \frac{\mathbf{x}^{\perp}}{|\mathbf{x}|^{2}}=\partial_{1}\left(\boldsymbol{\Gamma}(\mathbf{x}) \mathbf{e}_{2}\right)-\partial_{2}\left(\boldsymbol{\Gamma}(\mathbf{x}) \mathbf{e}_{1}\right)
\end{array}\right.
$$

where $\alpha_{1}$ and $\alpha_{2}$ are constants appearing in the definition 2.11 of the fundamental solution. The identities in (3.5) can be proved by straightforward computations.

The singular functions of this paper are constructed as linear combinations of functions given in (3.4). To motivate the construction, we temporarily assume that two inclusions $D_{1}$ and $D_{2}$ are symmetric with respect to both $x$ - and $y$-axes. If we write $\mathbf{h}_{1}=\left(h_{11}, h_{12}\right)^{T}$, then thanks to the symmetry of the inclusions and boundary conditions in (3.1), the following two functions are also solutions of $(3.1)$ for $j=1$ :

$$
\left[\begin{array}{c}
h_{11}(x,-y) \\
-h_{12}(x,-y)
\end{array}\right], \quad\left[\begin{array}{c}
-h_{11}(-x, y) \\
h_{12}(-x, y)
\end{array}\right] .
$$

By the uniqueness of the solution, we see that $\mathbf{h}_{1}$ has the following symmetric property with respect to $x$ - and $y$-axes:

$$
\left\{\begin{array}{l}
h_{11}(x, y)=h_{11}(x,-y)=-h_{11}(-x, y) \\
h_{12}(x, y)=-h_{12}(x,-y)=h_{12}(-x, y)
\end{array}\right.
$$


One can see that the following two functions have the same symmetry:

$$
\boldsymbol{\Gamma}\left(\mathbf{x}-\mathbf{p}_{1}\right) \mathbf{e}_{1}-\boldsymbol{\Gamma}\left(\mathbf{x}-\mathbf{p}_{2}\right) \mathbf{e}_{1}, \quad \frac{\mathbf{x}-\mathbf{p}_{1}}{\left|\mathbf{x}-\mathbf{p}_{1}\right|^{2}}+\frac{\mathbf{x}-\mathbf{p}_{2}}{\left|\mathbf{x}-\mathbf{p}_{2}\right|^{2}}
$$

So the first singular function $\mathbf{q}_{1}$ is constructed as a linear combination of these functions.

On the other hand, one can see in a similar way that $\mathbf{h}_{2}=\left(h_{21}, h_{22}\right)^{T}$ has the following symmetric property:

$$
\left\{\begin{array}{l}
h_{21}(x, y)=-h_{21}(x,-y)=h_{21}(-x, y), \\
h_{22}(x, y)=h_{22}(x,-y)=-h_{22}(-x, y)
\end{array}\right.
$$

and the following two functions have the same symmetry:

$$
\boldsymbol{\Gamma}\left(\mathbf{x}-\mathbf{p}_{1}\right) \mathbf{e}_{2}-\boldsymbol{\Gamma}\left(\mathbf{x}-\mathbf{p}_{2}\right) \mathbf{e}_{2}, \quad \frac{\left(\mathbf{x}-\mathbf{p}_{1}\right)^{\perp}}{\left|\mathbf{x}-\mathbf{p}_{1}\right|^{2}}+\frac{\left(\mathbf{x}-\mathbf{p}_{2}\right)^{\perp}}{\left|\mathbf{x}-\mathbf{p}_{2}\right|^{2}} .
$$

So $\mathbf{q}_{2}$ is constructed as a linear combination of these functions.

The singular functions of this paper are defined by

$$
\mathbf{q}_{1}(\mathbf{x}):=\boldsymbol{\Gamma}\left(\mathbf{x}-\mathbf{p}_{1}\right) \mathbf{e}_{1}-\boldsymbol{\Gamma}\left(\mathbf{x}-\mathbf{p}_{2}\right) \mathbf{e}_{1}+\alpha_{2} a\left(\frac{\mathbf{x}-\mathbf{p}_{1}}{\left|\mathbf{x}-\mathbf{p}_{1}\right|^{2}}+\frac{\mathbf{x}-\mathbf{p}_{2}}{\left|\mathbf{x}-\mathbf{p}_{2}\right|^{2}}\right)
$$

and

$$
\mathbf{q}_{2}(\mathbf{x}):=\boldsymbol{\Gamma}\left(\mathbf{x}-\mathbf{p}_{1}\right) \mathbf{e}_{2}-\boldsymbol{\Gamma}\left(\mathbf{x}-\mathbf{p}_{2}\right) \mathbf{e}_{2}-\alpha_{2} a\left(\frac{\left(\mathbf{x}-\mathbf{p}_{1}\right)^{\perp}}{\left|\mathbf{x}-\mathbf{p}_{1}\right|^{2}}+\frac{\left(\mathbf{x}-\mathbf{p}_{2}\right)^{\perp}}{\left|\mathbf{x}-\mathbf{p}_{2}\right|^{2}}\right),
$$

where $a$ is the number appearing in $(2.46)$. We emphasize that $a$ depends on $\epsilon$. In fact, we repeatedly use the fact that $a \approx \sqrt{\epsilon}$. The functions $\mathbf{q}_{j}$ satisfy $\mathcal{L}_{\lambda, \mu} \mathbf{q}_{j}=0$ in $\mathbb{R}^{2} \backslash\left\{\mathbf{p}_{1}, \mathbf{p}_{2}\right\}$, and

$$
\mathbf{q}_{j}(\mathbf{x})=O\left(|\mathbf{x}|^{-1}\right) \quad \text { as }|\mathbf{x}| \rightarrow \infty,
$$

as one can easily see. We emphasize that the symmetry of $D_{1} \cup D_{2}$ is not assumed here.

It will be proved later in Proposition 3.13 that

$$
\mathbf{h}_{j} \approx \frac{m_{j}}{\sqrt{\epsilon}} \mathbf{q}_{j}
$$

where $m_{1}$ and $m_{2}$ are constants defined by

$$
m_{1}:=\left[\left(\alpha_{1}-\alpha_{2}\right) \sqrt{2\left(\kappa_{1}+\kappa_{2}\right)}\right]^{-1}, \quad m_{2}:=\left[\left(\alpha_{1}+\alpha_{2}\right) \sqrt{2\left(\kappa_{1}+\kappa_{2}\right)}\right]^{-1} .
$$

So blow-up of $\nabla \mathbf{h}_{j}$ is captured by an explicit function $\frac{m_{j}}{\sqrt{\epsilon}} \nabla \mathbf{q}_{j}$. This is a crucial fact for investigating blow-up of $\nabla \mathbf{u}$ in this paper.

We now construct the third singular function $\mathbf{q}_{3}$ which approximates $\mathbf{h}_{3}$. For that we introduce $\boldsymbol{\Gamma}^{\perp}=\left(\Gamma_{i j}^{\perp}\right)_{i, j=1}^{2}$ which is defined by

$$
\boldsymbol{\Gamma}^{\perp}(\mathbf{x})=\alpha_{1} \arg (\mathbf{x})\left[\begin{array}{ll}
1 & 0 \\
0 & 1
\end{array}\right]-\frac{\alpha_{2}}{|\mathbf{x}|^{2}}\left[\begin{array}{cc}
-x_{1} x_{2} & -x_{2}^{2} \\
x_{1}^{2} & x_{1} x_{2}
\end{array}\right] .
$$


We emphasize that $\boldsymbol{\Gamma}^{\perp}$ is a multi-valued function since $\arg (\mathbf{x})$ is. So $\boldsymbol{\Gamma}^{\perp}$ is defined in $\mathbb{R}^{2}$ except a branch-cut starting from the origin.

Note that

$$
\boldsymbol{\Gamma}^{\perp}(\mathbf{x}) \mathbf{e}_{j}=\alpha_{1} \arg (\mathbf{x}) \mathbf{e}_{j}-\alpha_{2} x_{j} \nabla(\arg (\mathbf{x})), \quad j=1,2 .
$$

Since $\arg (\mathbf{x})$ is a harmonic function, we infer from Lemma 2.3 that $\boldsymbol{\Gamma}^{\perp}(\mathbf{x}) \mathbf{e}_{j}$ is a solution to the Lamé system (except on the branch-cut).

We now define the singular function $\mathbf{q}_{3}$ by

$$
\begin{aligned}
\mathbf{q}_{3}(\mathbf{x})= & m_{3}\left(\boldsymbol{\Gamma}^{\perp}\left(\mathbf{x}-\mathbf{p}_{1}\right)-\boldsymbol{\Gamma}^{\perp}\left(\mathbf{x}-\mathbf{c}_{1}\right)\right) \mathbf{e}_{1}+m_{3}\left(\boldsymbol{\Gamma}^{\perp}\left(\mathbf{x}-\mathbf{p}_{2}\right)-\boldsymbol{\Gamma}^{\perp}\left(\mathbf{x}-\mathbf{c}_{2}\right)\right) \mathbf{e}_{1} \\
& +m_{3} \alpha_{2} a\left(\frac{\left(\mathbf{x}-\mathbf{p}_{1}\right)^{\perp}}{\left|\mathbf{x}-\mathbf{p}_{1}\right|^{2}}-\frac{\left(\mathbf{x}-\mathbf{p}_{2}\right)^{\perp}}{\left|\mathbf{x}-\mathbf{p}_{2}\right|^{2}}\right)
\end{aligned}
$$

where

$$
m_{3}:=\left[\left(\alpha_{1}-\alpha_{2}\right)\left(\kappa_{1}+\kappa_{2}\right)\right]^{-1},
$$

and $\mathbf{c}_{1}$ and $\mathbf{c}_{2}$ are centers of the osculating disks $B_{1}$ and $B_{2}$, respectively. It is worth mentioning that $\boldsymbol{\Gamma}^{\perp}\left(\mathbf{x}-\mathbf{p}_{j}\right)-\boldsymbol{\Gamma}^{\perp}\left(\mathbf{x}-\mathbf{c}_{j}\right)$ is well-defined in $\mathbb{R}^{2}$ except a branch-cut connecting $\mathbf{p}_{j}$ and $\mathbf{c}_{j}$. So, $\mathbf{q}_{3}$ is well-defined and a solution of the Lamé system in $D^{e}$. We will show in Lemma 3.11 that $\mathbf{q}_{3}$ has the same local behavior as $\mathbf{h}_{3}$.

In subsections to follow we derive technical estimates of $\mathbf{q}_{j}$ and its derivatives which will be used in later sections.

\subsection{Estimates of the function $\zeta$}

We show in the next subsection that the singular function $\mathbf{q}_{j}(j=1,2)$ can be nicely represented using the function $q_{B}$ given in (3.3). In fact, it is slightly more convenient to use the function $\zeta(\mathbf{x})$ defined by

$$
\zeta(\mathbf{x})=2 \pi q_{B}(\mathbf{x})
$$

The following lemma collects estimates for the function $\zeta$ to be used in the next subsection. Some of the estimates are essentially proved in [1]. However, in that paper the estimates are not explicitly written and derivations of estimates are smeared in other proofs. So we include proofs.

Lemma 3.1. (i) Let $\Pi_{L}$ be the narrow region defined in 2.55). It holds that

$$
|\zeta(\mathbf{x})| \lesssim \sqrt{\epsilon}, \quad \mathbf{x} \in \Pi_{L},
$$

and

$$
\left|\partial_{1} \zeta(\mathbf{x})\right| \lesssim \frac{\sqrt{\epsilon}}{\epsilon+y^{2}}, \quad\left|\partial_{2} \zeta(\mathbf{x})\right| \lesssim \frac{\sqrt{\epsilon}|y|}{\epsilon+y^{2}}, \quad \mathbf{x}=(x, y) \in \Pi_{L} .
$$

(ii) Let $\mathbf{x}_{j}(y)$ be the defining functions for $\partial D_{j}$ for $j=1,2$ as given in 2.52). For $|y|<L$ and $j=1,2$, we have

$$
\begin{aligned}
\left|\zeta\left(\mathbf{x}_{j}(y)\right)-\zeta\right|_{\partial B_{j}} \mid & \lesssim \sqrt{\epsilon}\left(\left|\omega_{j}\right||y|+|y|^{2}\right), \\
\left|\frac{d}{d y} \zeta\left(\mathbf{x}_{j}(y)\right)\right| & \lesssim \sqrt{\epsilon} \\
\left|\frac{d^{2}}{d y^{2}} \zeta\left(\mathbf{x}_{j}(y)\right)\right| & \lesssim \frac{\sqrt{\epsilon}}{\epsilon+y^{2}}
\end{aligned}
$$


Proof. (i) Since $\mathbf{p}_{1}=(-a, 0)$ and $\mathbf{p}_{2}=(a, 0)$, we can rewrite $\zeta(\mathbf{x})$ as

$$
\zeta(\mathbf{x})=\frac{1}{2} \ln \frac{(x+a)^{2}+y^{2}}{(x-a)^{2}+y^{2}}=\frac{1}{2} \ln \left(1+\frac{4 a x}{(x-a)^{2}+y^{2}}\right) .
$$

Since $a \approx \sqrt{\epsilon}, \epsilon+y^{2} \approx(x \pm a)^{2}+y^{2}$, and $|x| \lesssim \epsilon+y^{2}$ for $(x, y) \in \Pi_{L}$, we obtain

$$
|\zeta(\mathbf{x})|=\frac{1}{2} \ln (1+O(\sqrt{\epsilon}))=O(\sqrt{\epsilon}) \quad \text { for } \mathbf{x} \in \Pi_{L},
$$

which yields (3.18).

Assume $\mathbf{x}=(x, y) \in \Pi_{L}$. Since

$$
|x| \lesssim \epsilon+y^{2} \quad \text { and } \quad \epsilon+y^{2} \lesssim(x \pm a)^{2}+y^{2}
$$

we have from the first identity in $(3.23)$ that

$$
\begin{aligned}
\left|\partial_{1} \zeta(\mathbf{x})\right| & =\left|\frac{x+a}{(x+a)^{2}+y^{2}}-\frac{x-a}{(x-a)^{2}+y^{2}}\right| \\
& =\left|\frac{2 a\left(a^{2}-x^{2}+y^{2}\right)}{\left((x+a)^{2}+y^{2}\right)\left((x+a)^{2}+y^{2}\right)}\right| \lesssim \frac{\sqrt{\epsilon}\left(\epsilon+y^{2}\right)}{\left(\epsilon+y^{2}\right)^{2}} \lesssim \frac{\sqrt{\epsilon}}{\epsilon+y^{2}},
\end{aligned}
$$

and

$$
\begin{aligned}
\left|\partial_{2} \zeta(\mathbf{x})\right| & =|y|\left|\frac{1}{(x+a)^{2}+y^{2}}-\frac{1}{(x-a)^{2}+y^{2}}\right| \\
& \lesssim|y|\left|\frac{4 a x}{\left((x+a)^{2}+y^{2}\right)\left((x-a)^{2}+y^{2}\right)}\right| \lesssim \frac{\sqrt{\epsilon}|y||x|}{\left(\epsilon+y^{2}\right)^{2}} \lesssim \frac{\sqrt{\epsilon}|y|}{\epsilon+y^{2}} .
\end{aligned}
$$

So $(3.19)$ is proved.

(ii) We now prove $(3.20)$. For simplicity, we assume $j=1$. Let us write the boundary $\partial B_{1}$ of the osculating disk $B_{1}$ as $\left(-f_{B}(y), y\right)$ for $|y|<L$. Recall from $(2.53)$ that

$$
f_{1}(y)-f_{B}(y)=\frac{1}{3 !} \omega_{1} y^{3}+O\left(y^{4}\right) .
$$

From 3.23 , we have

$$
\begin{aligned}
\left|\zeta\left(\mathbf{x}_{1}(y)\right)-\zeta\right|_{\partial B_{1}} \mid & =\frac{1}{2}\left|\ln \left(1-\frac{4 a f_{1}(y)}{\left(f_{1}(y)+a\right)^{2}+y^{2}}\right)-\ln \left(1-\frac{4 a f_{B}(y)}{\left(f_{B}(y)+a\right)^{2}+y^{2}}\right)\right| \\
& =\frac{1}{2}\left|\ln \left(1-4 a \frac{\eta_{1}(y)}{\eta_{2}(y)}\right)\right|
\end{aligned}
$$

where

$$
\begin{aligned}
& \eta_{1}(y)=\frac{f_{1}(y)}{\left(f_{1}(y)+a\right)^{2}+y^{2}}-\frac{f_{B}(y)}{\left(f_{B}(y)+a\right)^{2}+y^{2}}, \\
& \eta_{2}(y)=1-\frac{4 a f_{B}(y)}{\left(f_{B}(y)+a\right)^{2}+y^{2}} .
\end{aligned}
$$

Since $a \approx \sqrt{\epsilon}, f_{B}(y) \approx \epsilon+y^{2}$, and $\left(f_{B}(y)+a\right)^{2}+y^{2} \approx \epsilon+y^{2}$, we see that

$$
\left|\eta_{2}(y)\right| \approx 1 \text { for }|y|<L
$$


From (3.24) and the facts that $f_{1} \approx \epsilon+y^{2}, f_{B} \approx \epsilon+y^{2}$ and $a \approx \sqrt{\epsilon}$, we have, for $|y|<L$,

$$
\begin{aligned}
\left|\eta_{1}(y)\right| & =\left|\frac{\left(f_{1}(y)-f_{B}(y)\right)\left(y^{2}-f_{1}(y) f_{B}(y)+a^{2}\right)}{\left(\left(f_{1}(y)+a\right)^{2}+y^{2}\right)\left(\left(f_{B}(y)+a\right)^{2}+y^{2}\right)}\right| \\
& \lesssim \frac{\left(\omega_{1}|y|^{3}+y^{4}\right)\left(y^{2}+\left(\epsilon+y^{2}\right)^{2}+\epsilon\right)}{\left(\epsilon+y^{2}\right)^{2}} \\
& \lesssim\left|\omega_{1}\right||y|+y^{2} .
\end{aligned}
$$

Since $a \approx \sqrt{\epsilon}$, it follows from $3.25,3.28$, and 3.29 that

$$
\left|\zeta\left(\mathbf{x}_{1}(y)\right)-\zeta\right|_{\partial B_{1}} \mid \lesssim \sqrt{\epsilon}\left(\left|\omega_{1}\right||y|+y^{2}\right)
$$

Therefore 3.20$)$ is proved.

We now prove (3.21) and $(3.22)$ for $j=1$. The cases for $j=2$ can be handled similarly. In view of (3.23), we have

$$
\begin{aligned}
\frac{d}{d y} \zeta\left(\mathbf{x}_{1}(y)\right)= & \frac{d}{d y} \zeta\left(-f_{1}(y), y\right) \\
= & \left(\frac{-f_{1}(y)+a}{\left(-f_{1}(y)+a\right)^{2}+y^{2}}-\frac{y}{\left(-f_{1}(y)-a\right)^{2}+y^{2}}\right)\left(-f_{1}^{\prime}(y)\right) \\
& \quad+\frac{y}{\left(-f_{1}(y)+a\right)^{2}+y^{2}}-\frac{y}{\left(-f_{1}(y)-a\right)^{2}+y^{2}} \\
= & 2 a \frac{N(y)}{D_{+}(y) D_{-}(y)},
\end{aligned}
$$

where $N$ and $D_{ \pm}$are given by

$$
\begin{aligned}
N(y) & :=(-1)\left(a^{2}-f_{1}(y)^{2}+y^{2}\right) f_{1}^{\prime}(y)+2 f_{1}(y) y, \\
D_{ \pm}(y) & :=\left(-f_{1}(y) \pm a\right)^{2}+y^{2} .
\end{aligned}
$$

It is easy to see that

$$
D_{ \pm}(y) \approx \epsilon+y^{2}
$$

As consequences of 2.48 and 2.53), we have

$$
a^{2}=\frac{2 \epsilon}{\kappa_{1}+\kappa_{2}}+O\left(\epsilon^{2}\right), \quad f_{1}(y)=\frac{\kappa_{1}}{\kappa_{1}+\kappa_{2}} \epsilon+\frac{1}{2} \kappa_{1} y^{2}+O\left(\epsilon^{2}+y^{3}\right),
$$

and hence

$$
\begin{aligned}
N(y)= & (-1)\left(\frac{2 \epsilon}{\kappa_{1}+\kappa_{2}}+y^{2}\right) \kappa_{1} y \\
& +2\left(\frac{\kappa_{1}}{\kappa_{1}+\kappa_{2}} \epsilon+\frac{1}{2} \kappa_{1} y^{2}\right) y+O\left(\epsilon y^{3}+\epsilon^{2} y+y^{4}\right) \\
= & O\left(\epsilon y^{2}+\epsilon^{2} y+y^{4}\right) .
\end{aligned}
$$

Then, from 3.30 and the fact that $a \approx \sqrt{\epsilon}$, we have

$$
\left|\frac{d}{d y} \zeta\left(\mathbf{x}_{1}(y)\right)\right| \lesssim a \frac{\epsilon y^{2}+\epsilon^{2} y+y^{4}}{\left(\epsilon+y^{2}\right)^{2}} \lesssim \sqrt{\epsilon}
$$


So, 3.21 is proved.

Now let us consider (3.22). We have

$$
\begin{aligned}
\frac{d^{2}}{d y^{2}} \zeta\left(\mathbf{x}_{1}(y)\right) & =\frac{d^{2}}{d y^{2}} \zeta\left(-f_{1}(y), y\right)=\frac{d}{d y}\left(2 a \frac{N(y)}{D_{+}(y) D_{-}(y)}\right) \\
& =2 a \frac{N^{\prime}(y)}{D_{+}(y) D_{-}(y)}-2 a \frac{N(y)\left(D_{+}\right)^{\prime}(y)}{\left(D_{+}(y)\right)^{2} D_{-}(y)}-2 a \frac{N(y)\left(D_{-}\right)^{\prime}(y)}{D_{+}(y)\left(D_{-}(y)\right)^{2}} .
\end{aligned}
$$

We also have

$$
\begin{aligned}
N^{\prime}(y) & =-a^{2} f_{1}^{\prime \prime}+2 f_{1}\left(f_{1}^{\prime}\right)^{2}+\left(f_{1}\right)^{2} f_{1}^{\prime \prime}-y^{2} f_{1}^{\prime \prime}+2 f_{1}, \\
\left(D_{ \pm}\right)^{\prime}(y) & =2\left(-f_{1} \pm a\right)\left(-f_{1}^{\prime}\right)+2 y .
\end{aligned}
$$

Since $f_{1}(y) \approx \epsilon+y^{2}, f_{1}^{\prime}(y)=O(y)$ and $f_{1}^{\prime \prime}(y)=O(1)$, we have

$$
\left|N^{\prime}(y)\right| \lesssim \epsilon+y^{2}, \quad\left|\left(D_{ \pm}\right)^{\prime}(y)\right| \lesssim|y| .
$$

Then, from (3.31)-3.33 and the fact that $a \approx \sqrt{\epsilon}$, we obtain

$$
\left|\frac{d^{2}}{d y^{2}} \zeta\left(\mathbf{x}_{1}(y)\right)\right| \lesssim a\left(\frac{\epsilon+y^{2}}{\left(\epsilon+y^{2}\right)^{2}}+\frac{\left(\epsilon y^{2}+\epsilon^{2} y+y^{4}\right)|y|}{\left(\epsilon+y^{2}\right)^{3}}\right) \lesssim \frac{\sqrt{\epsilon}}{\epsilon+y^{2}} .
$$

The proof is completed.

\subsection{Estimates of singular functions}

This subsection is to derive estimates of the singular function $\mathbf{q}_{j}$ in the narrow region $\Pi_{L}$ and on $\partial D_{1} \cup \partial D_{2}$, which will be used in the later part of the paper.

We begin by showing that singular functions can be explicitly represented by the function $\zeta$ introduced in the previous subsection. Set

$$
A_{\zeta}(\mathbf{x}):=\left[1-\frac{\sinh ^{2} \zeta(\mathbf{x})}{a^{2}} y^{2}\right]^{1 / 2}, \quad \mathbf{x}=(x, y)
$$

If $\mathbf{x}=(x, y) \in \Pi_{L}$, then it holds by (3.18) that $|\zeta(\mathbf{x})| \lesssim \sqrt{\epsilon}$. Since $a \approx \sqrt{\epsilon}$ by (2.48), we have

$$
\frac{\sinh ^{2} \zeta(\mathbf{x})}{a^{2}} \lesssim 1
$$

So there exists a constant $0<L_{0}<L$ (independent of $\epsilon$ ) such that

$$
1-\frac{\sinh ^{2} \zeta(\mathbf{x})}{a^{2}} y^{2} \geq \frac{1}{2}, \quad \mathbf{x}=(x, y) \in \Pi_{L_{0}} .
$$

Note that

$$
A_{\zeta}(\mathbf{x})=1+O\left(y^{2}\right), \quad \mathbf{x}=(x, y) \in \Pi_{L_{0}} .
$$

Singular functions $\mathbf{q}_{j}$ can be represented in terms of $\zeta$ as follows 
Proposition 3.2. Let $\mathbf{q}_{i}(\mathbf{x})=\left(q_{i 1}(\mathbf{x}), q_{i 2}(\mathbf{x})\right)^{T}$ for $i=1,2$. If $\mathbf{x} \in \Pi_{L_{0}}$, then $q_{i j}$ are given by

$$
\begin{aligned}
q_{11}(\mathbf{x}) & =\alpha_{1} \zeta(\mathbf{x})-\alpha_{2} A_{\zeta}(\mathbf{x}) \sinh \zeta(\mathbf{x}) \\
q_{12}(\mathbf{x})=q_{21}(\mathbf{x}) & =\alpha_{2} a^{-1} y \sinh ^{2} \zeta(\mathbf{x}) \\
q_{22}(\mathbf{x}) & =\alpha_{1} \zeta(\mathbf{x})+\alpha_{2} A_{\zeta}(\mathbf{x}) \sinh \zeta(\mathbf{x})
\end{aligned}
$$

Proof. From the definition (3.8) of $\mathbf{q}_{1}$ and the first identity in $(3.23)$, we have

$$
\begin{aligned}
q_{11}(\mathbf{x})= & \alpha_{1} \zeta(\mathbf{x})-\alpha_{2}\left[\frac{(x+a)^{2}}{(x+a)^{2}+y^{2}}-\frac{(x-a)^{2}}{(x-a)^{2}+y^{2}}\right] \\
& +\alpha_{2} a\left[\frac{x+a}{(x+a)^{2}+y^{2}}+\frac{x-a}{(x-a)^{2}+y^{2}}\right] \\
= & \alpha_{1} \zeta(\mathbf{x})-\alpha_{2}\left[\frac{x(x+a)}{(x+a)^{2}+y^{2}}-\frac{x(x-a)}{(x-a)^{2}+y^{2}}\right] \\
= & \alpha_{1} \zeta(\mathbf{x})-\alpha_{2} \frac{2 a x\left(a^{2}-x^{2}+y^{2}\right)}{\left((x-a)^{2}+y^{2}\right)\left((x+a)^{2}+y^{2}\right)}
\end{aligned}
$$

for $\mathbf{x} \in \mathbb{R}^{2} \backslash\left\{\mathbf{p}_{1}, \mathbf{p}_{2}\right\}$.

Thanks to the first identity in (3.23) again, we have

$$
\begin{aligned}
\sinh \zeta(\mathbf{x}) & =\frac{1}{2}\left(\sqrt{\frac{(x+a)^{2}+y^{2}}{(x-a)^{2}+y^{2}}}-\sqrt{\frac{(x-a)^{2}+y^{2}}{(x+a)^{2}+y^{2}}}\right) \\
& =\frac{2 a x}{\sqrt{(x-a)^{2}+y^{2}} \sqrt{(x+a)^{2}+y^{2}}}
\end{aligned}
$$

for $\mathbf{x} \in \mathbb{R}^{2} \backslash\left\{\mathbf{p}_{1}, \mathbf{p}_{2}\right\}$. Then straightforward computations yield

$$
A_{\zeta}(\mathbf{x})=\frac{a^{2}-x^{2}+y^{2}}{\sqrt{\left((x+a)^{2}+y^{2}\right)\left((x-a)^{2}+y^{2}\right)}}, \quad \mathbf{x} \in \Pi_{L_{0}} .
$$

This together with 3.40 yields (3.37).

The identity 3.38 can be proved similarly. In fact, one can see that

$$
\begin{aligned}
q_{12}(\mathbf{x})= & -\alpha_{2}\left[\frac{(x+a) y}{(x+a)^{2}+y^{2}}-\frac{(x-a) y}{(x-a)^{2}+y^{2}}\right] \\
& +\alpha_{2} a\left[\frac{y}{(x+a)^{2}+y^{2}}+\frac{y}{(x-a)^{2}+y^{2}}\right] \\
= & \alpha_{2} \frac{4 a x^{2} y}{\left((x+a)^{2}+y^{2}\right)\left((x-a)^{2}+y^{2}\right)},
\end{aligned}
$$

and (3.38) follows from (3.41).

Similarly one can show that $q_{21}=q_{12}$ and 3.39 ) hold. We omit the proof.

Proposition 3.2 already reveals an important property of the singular functions. They are almost constant near the points $\mathbf{z}_{1}$ and $\mathbf{z}_{2}$. This can be seen more clearly if two osculating disks have the same radii. 
Lemma 3.3. Assume $B_{1}$ and $B_{2}$ have the same radii $r_{0}$. Then it holds for $\mathbf{x} \in \partial B_{i}$, $i=1,2$, that

$$
\begin{aligned}
& \mathbf{q}_{1}(\mathbf{x})=\left(\frac{\sqrt{\epsilon}}{m_{1}}+t_{1}\right) \frac{(-1)^{i}}{2} \Psi_{1}+\alpha_{2} \frac{a}{r_{0}^{2}}\left[\begin{array}{l}
x \\
y
\end{array}\right], \\
& \mathbf{q}_{2}(\mathbf{x})=\left(\frac{\sqrt{\epsilon}}{m_{2}}+t_{2}\right) \frac{(-1)^{i}}{2} \Psi_{2}+\alpha_{2} \frac{a}{r_{0}^{2}}\left[\begin{array}{l}
y \\
x
\end{array}\right]
\end{aligned}
$$

where $m_{j}$ are constants defined by (3.12) and $t_{j}$ are constants satisfying

$$
\left|t_{j}\right| \leq C\left(\alpha_{1}+\alpha_{2}\right) \epsilon^{3 / 2}
$$

for some constant $C$ independent of $\left(\alpha_{1}, \alpha_{2}\right)$, or equivalently, independent of $(\lambda, \mu)$, as well as $\epsilon$.

Proof. If $r_{1}=r_{2}=r_{0}, 2.47$ reads

$$
a=\frac{\sqrt{\epsilon\left(4 r_{0}+\epsilon\right)}}{2}
$$

We see from 3.23 that the constant value $\zeta(\mathbf{x})$ on $\partial B_{j}$ are as follows:

$$
\left.\zeta\right|_{\partial B_{i}}=(-1)^{i} \sinh ^{-1}\left(a / r_{0}\right), \quad i=1,2 .
$$

Let $s=\sinh ^{-1}\left(a / r_{0}\right)$. Note that $a=r_{0} \sinh s$. Then it follows from 3.47 that

$$
r_{0} \cosh s=\sqrt{r_{0}^{2}+a^{2}}=r_{0}+\epsilon / 2 .
$$

Since the center of $\partial B_{i}$ is $(-1)^{i}\left(r_{0}+\epsilon / 2,0\right)$, we have

$$
\partial B_{i}=\left\{(x, y) \in \mathbb{R}^{2}:\left(x-(-1)^{i} r_{0} \cosh s\right)^{2}+y^{2}=r_{0}^{2}\right\} .
$$

So, for $(x, y) \in \partial B_{i}$, we obtain

$$
\begin{aligned}
(x \pm a)^{2}+y^{2} & =x^{2} \pm 2 x r_{0} \sinh s+r_{0}^{2} \sinh ^{2} s+y^{2} \\
& =\left(x-(-1)^{i} r_{0} \cosh s\right)^{2}+y^{2}-r_{0}^{2}+(-1)^{i} 2 x r_{0} \cosh s \pm 2 x r_{0} \sinh s \\
& =\left((-1)^{i} \cosh s \pm \sinh s\right) 2 r_{0} x,
\end{aligned}
$$

and

$$
\begin{aligned}
a^{2}-x^{2}+y^{2} & =\left(x-(-1)^{i} r_{0} \cosh s\right)^{2}+y^{2}-r_{0}^{2}-2 x^{2}+(-1)^{i} 2 x r_{0} \cosh s \\
& =\left((-1)^{i} r_{0} \cosh s-x\right) 2 x=\left((-1)^{i}\left(r_{0}+\epsilon / 2\right)-x\right) 2 x .
\end{aligned}
$$

Then, for $(x, y) \in \partial B_{i}$, we have

$$
\begin{aligned}
& \frac{2 a x}{\sqrt{(x-a)^{2}+y^{2}} \sqrt{(x+a)^{2}+y^{2}}}=(-1)^{i} \frac{a}{r_{0}}, \\
& \frac{a^{2}-x^{2}+y^{2}}{\sqrt{\left((x+a)^{2}+y^{2}\right)\left((x-a)^{2}+y^{2}\right)}}=1+\frac{\epsilon}{2 r_{0}}-(-1)^{i} \frac{x}{r_{0}} .
\end{aligned}
$$


Note that $\left.\zeta\right|_{\partial B_{i}}=(-1)^{i}$ s. So we obtain from 3.40 and 3.50 that

$$
q_{11}(\mathbf{x})=(-1)^{i} \alpha_{1} s-\alpha_{2}(-1)^{i}\left(1+\frac{\epsilon}{2 r_{0}}\right) \frac{a}{r_{0}}+\alpha_{2} \frac{a}{r_{0}} \frac{x}{r_{0}}, \quad \mathbf{x} \in \partial B_{i} .
$$

Since $a=\sqrt{r_{0} \epsilon}+O\left(\epsilon^{3 / 2}\right), s=\sqrt{\epsilon / r_{0}}+O\left(\epsilon^{3 / 2}\right)$, and $m_{1}=\sqrt{r_{0}} /\left[2\left(\alpha_{1}-\alpha_{2}\right)\right]$, it follows that

$$
\begin{aligned}
q_{11}(\mathbf{x}) & =(-1)^{i}\left(\left(\alpha_{1}-\alpha_{2}\right) \sqrt{\epsilon / r_{0}}+\left(\alpha_{1}+\alpha_{2}\right) O\left(\epsilon^{3 / 2}\right)\right)+\alpha_{2} \frac{a}{r_{0}^{2}} x \\
& =(-1)^{i}\left(\frac{1}{2} \frac{\sqrt{\epsilon}}{m_{1}}+\left(\alpha_{1}+\alpha_{2}\right) O\left(\epsilon^{3 / 2}\right)\right)+\alpha_{2} \frac{a}{r_{0}^{2}} x, \quad \mathbf{x} \in \partial B_{1} .
\end{aligned}
$$

We also obtain from 3.43 and 3.49 that

$$
q_{12}(\mathbf{x})=\alpha_{2} \frac{a}{r_{0}^{2}} y, \quad \mathbf{x} \in \partial B_{1} \cup \partial B_{2} .
$$

This proves (3.44). One can prove 3.45 similarly.

We have the following lemma in $\Pi_{L_{0}}$.

Lemma 3.4. We have, for $\mathrm{x} \in \Pi_{L_{0}}$,

$$
\left|\partial_{1} q_{11}(\mathbf{x})\right|+\left|\partial_{1} q_{22}(\mathbf{x})\right| \lesssim \frac{\sqrt{\epsilon}}{\epsilon+y^{2}}
$$

and

$$
\left|\partial_{2} q_{11}(\mathbf{x})\right|+\left|\partial_{2} q_{22}(\mathbf{x})\right|+\left|\nabla q_{12}(\mathbf{x})\right| \lesssim \frac{\sqrt{\epsilon}|y|}{\epsilon+y^{2}}+\sqrt{\epsilon} .
$$

Proof. We only consider $\mathbf{q}_{1}$. Estimates for $\mathbf{q}_{2}$ can be obtained similarly.

First we consider $\partial_{1} q_{11}(\mathbf{x})$. By (3.37), we have

$$
\begin{aligned}
\partial_{1} q_{11}(\mathbf{x})= & \alpha_{1} \partial_{1} \zeta(\mathbf{x})-\alpha_{2} \cosh (\zeta(\mathbf{x})) A_{\zeta}(\mathbf{x}) \partial_{1} \zeta(\mathbf{x}) \\
& +\alpha_{2} \sinh ^{2}(\zeta(\mathbf{x})) \cosh (\zeta(\mathbf{x})) \frac{y^{2}}{a^{2}} A_{\zeta}(\mathbf{x})^{-1} \partial_{1} \zeta(\mathbf{x})
\end{aligned}
$$

Thanks to 3.35, we have

$$
\left|A_{\zeta}(\mathbf{x})\right| \approx 1
$$

Then, using the fact that $a \approx \sqrt{\epsilon}$, we obtain

$$
\left|\partial_{1} q_{11}(\mathbf{x})\right| \lesssim\left|\partial_{1} \zeta(\mathbf{x})\right|+|\zeta(\mathbf{x})|^{2} \frac{y^{2}}{\epsilon}\left|\partial_{1} \zeta(\mathbf{x})\right| .
$$

We then infer from 3.18 and 3.19 that

$$
\left|\partial_{1} q_{11}(\mathbf{x})\right| \lesssim \frac{\sqrt{\epsilon}}{\epsilon+y^{2}}+\epsilon \frac{y^{2}}{\epsilon} \frac{\sqrt{\epsilon}}{\epsilon+y^{2}} \lesssim \frac{\sqrt{\epsilon}}{\epsilon+y^{2}} .
$$

This proves 3.51. 
To prove 3.52 for $\mathbf{q}_{1}$, we compute using (3.37) and (3.38)

$$
\begin{aligned}
\partial_{2} q_{11}(\mathbf{x})= & \alpha_{1} \partial_{2} \zeta(\mathbf{x})-\alpha_{2} \cosh (\zeta(\mathbf{x})) A_{\zeta}(\mathbf{x}) \partial_{2} \zeta(\mathbf{x}) \\
& +\alpha_{2} \sinh (\zeta(\mathbf{x})) A_{\zeta}(\mathbf{x})^{-1}\left(\sinh \zeta(\mathbf{x}) \partial_{2} \zeta(\mathbf{x}) \frac{y^{2}}{a^{2}}+\sinh ^{2} \zeta(\mathbf{x}) \frac{y}{a^{2}}\right)
\end{aligned}
$$

and

$$
\begin{aligned}
& \partial_{1} q_{12}(\mathbf{x})=2 \alpha_{2} a y \sinh \zeta(\mathbf{x}) \cosh \zeta(\mathbf{x}) \partial_{1} \zeta(\mathbf{x}), \\
& \partial_{2} q_{12}(\mathbf{x})=2 \alpha_{2} a y \sinh \zeta(\mathbf{x}) \cosh \zeta(\mathbf{x}) \partial_{2} \zeta(\mathbf{x})+\alpha_{2} a \sinh ^{2} \zeta(\mathbf{x})
\end{aligned}
$$

So, (3.52 can be proved in the same way as above. The proof is completed.

Let $\mathbf{h}_{1}=\left(h_{11}, h_{12}\right)^{T}$ be the solution to 3.1 for $j=1$. Then, we have $\mathbf{h}_{1}\left(\mathbf{x}_{2}(y)\right)-$ $\mathbf{h}_{1}\left(\mathbf{x}_{1}(y)\right)=(1,0)^{T}$. Since $\left|\mathbf{x}_{2}(0)-\mathbf{x}_{1}(0)\right|=\epsilon$, one can expect

$$
\left\{\begin{array}{l}
\partial_{1} h_{11}(0,0)=\epsilon^{-1}+O(1), \\
\left|\partial_{2} h_{11}(0,0)\right|+\left|\partial_{1} h_{12}(0,0)\right|+\left|\partial_{2} h_{12}(0,0)\right| \lesssim 1 .
\end{array}\right.
$$

One can expect a similar behavior for $\mathbf{h}_{2}$ as well. We now show that $\frac{m_{j}}{\sqrt{\epsilon}} \mathbf{q}_{j}$ has the exactly same behavior as $\epsilon \rightarrow 0$.

Lemma 3.5. It holds for small $\epsilon>0$ that

$$
\left\{\begin{array}{l}
\partial_{1} q_{11}(0,0)=\frac{1}{m_{1} \sqrt{\epsilon}}+O(\sqrt{\epsilon}), \\
\left|\partial_{2} q_{11}(0,0)\right|+\left|\partial_{1} q_{12}(0,0)\right|+\left|\partial_{2} q_{12}(0,0)\right| \lesssim \sqrt{\epsilon},
\end{array}\right.
$$

and

$$
\left\{\begin{array}{l}
\partial_{1} q_{22}(0,0)=\frac{1}{m_{2} \sqrt{\epsilon}}+O(\sqrt{\epsilon}), \\
\left|\partial_{1} q_{21}(0,0)\right|+\left|\partial_{2} q_{21}(0,0)\right|+\left|\partial_{2} q_{22}(0,0)\right| \lesssim \sqrt{\epsilon} .
\end{array}\right.
$$

Proof. Since $\partial_{1} \zeta(0,0)=2 / a$ and $\zeta(0,0)=0$, it follows from (3.53) that

$$
\partial_{1} q_{11}(0,0)=\frac{2\left(\alpha_{1}-\alpha_{2}\right)}{a} .
$$

Since $a=\sqrt{2 \epsilon} / \sqrt{\left(\kappa_{1}+\kappa_{2}\right)}+O\left(\epsilon^{3 / 2}\right)$, the first equality in 3.56 follows. From 3.52 , we have

$$
\left|\partial_{2} q_{11}(0,0)\right|+\left|\partial_{1} q_{12}(0,0)\right|+\left|\partial_{2} q_{12}(0,0)\right| \lesssim \sqrt{\epsilon} .
$$

This proves 3.56) (3.57) can be proved similarly.

Lemma 3.6. For $j=1,2$, we have

$$
\left\|\mathbf{q}_{j}\right\|_{L^{\infty}\left(D^{e} \backslash \Pi_{L_{0}}\right)}+\left\|\nabla \mathbf{q}_{j}\right\|_{L^{\infty}\left(D^{e} \backslash \Pi_{L_{0}}\right)} \lesssim \sqrt{\epsilon}
$$

Proof. We only prove 3.58 for $j=1$. The same proof applies to the case when $j=2$.

Recall that

$$
\mathbf{q}_{1}(\mathbf{x})=\boldsymbol{\Gamma}\left(\mathbf{x}-\mathbf{p}_{1}\right) \mathbf{e}_{1}-\boldsymbol{\Gamma}\left(\mathbf{x}-\mathbf{p}_{2}\right) \mathbf{e}_{1}+\alpha_{2} a\left(\frac{\mathbf{x}-\mathbf{p}_{1}}{\left|\mathbf{x}-\mathbf{p}_{1}\right|^{2}}+\frac{\mathbf{x}-\mathbf{p}_{2}}{\left|\mathbf{x}-\mathbf{p}_{2}\right|^{2}}\right) .
$$


Note that if $\mathbf{x} \in D^{e} \backslash \Pi_{L_{0}}$, then $1 \lesssim|\mathbf{x}-\mathbf{p}|$ for all $\mathbf{p}$ lying on the line segment $\overline{\mathbf{p}_{1} \mathbf{p}_{2}}$. Since $a \approx \sqrt{\epsilon}$, the second term on the right-hand side of the above and its derivative is less than $\sqrt{\epsilon}$.

One can easily show that the first term also satisfies the same estimate. In fact, by the mean value theorem, we have

$$
\left|\boldsymbol{\Gamma}\left(\mathbf{x}-\mathbf{p}_{1}\right)-\boldsymbol{\Gamma}\left(\mathbf{x}-\mathbf{p}_{2}\right)\right| \lesssim\left|\nabla \boldsymbol{\Gamma}\left(\mathbf{x}-\mathbf{p}_{*}\right)\right|\left|\mathbf{p}_{1}-\mathbf{p}_{2}\right|
$$

for some $\mathbf{p}_{*}$ on $\overline{\mathbf{p}_{1} \mathbf{p}_{2}}$. We also have

$$
\left|\nabla\left(\boldsymbol{\Gamma}\left(\mathbf{x}-\mathbf{p}_{1}\right)-\boldsymbol{\Gamma}\left(\mathbf{x}-\mathbf{p}_{2}\right)\right)\right| \lesssim\left|\nabla^{2} \boldsymbol{\Gamma}\left(\mathbf{x}-\mathbf{p}_{* *}\right)\right|\left|\mathbf{p}_{1}-\mathbf{p}_{2}\right|
$$

for some $\mathbf{p}_{* *}$ on $\overline{\mathbf{p}_{1} \mathbf{p}_{2}}$. Since $\left|\mathbf{p}_{1}-\mathbf{p}_{2}\right|=2 a \approx \sqrt{\epsilon},(3.58)$ follows.

As a corollary, we have the following estimate for $\nabla \mathbf{q}_{j}$.

Corollary 3.7. For $j=1,2$, we have

$$
\left\|\nabla \mathbf{q}_{j}\right\|_{L^{\infty}\left(D^{e}\right)} \approx \epsilon^{-1 / 2}
$$

Proof. The upper estimate $\left\|\nabla \mathbf{q}_{j}\right\|_{L^{\infty}\left(D^{e}\right)} \lesssim \epsilon^{-1 / 2}$ is a consequence of Lemma 3.4 and 3.6 . and the lower one is that of Lemma 3.5 .

We have the following lemma on $\partial D_{1} \cup \partial D_{2}$.

Lemma 3.8. Let $\mathbf{x}_{k}(y)$ be the defining functions for $\partial D_{k}$ for $k=1,2$ as given in 2.52). For $|y|<L_{0}$, the following holds:

$$
\begin{aligned}
q_{11}\left(\mathbf{x}_{k}(y)\right) & =(-1)^{k}\left(\alpha_{1}-\alpha_{2}\right) \kappa_{k} a+O(E), \\
q_{12}\left(\mathbf{x}_{k}(y)\right)= & q_{21}\left(\mathbf{x}_{k}(y)\right)=\alpha_{2} \kappa_{k}^{2} a y+O(|y| E), \\
& q_{22}\left(\mathbf{x}_{k}(y)\right)=(-1)^{k}\left(\alpha_{1}+\alpha_{2}\right) \kappa_{k} a+O(E),
\end{aligned}
$$

where

$$
E:=\epsilon^{3 / 2}+\sqrt{\epsilon} y^{2}+\tau \sqrt{\epsilon}|y| .
$$

Proof. We see from 3.23 that

$$
\left.\zeta\right|_{\partial B_{k}}=(-1)^{i} \sinh ^{-1}\left(\kappa_{k} a\right)=(-1)^{i} \kappa_{k} a+O\left(a^{3}\right) .
$$

Since $a \approx \sqrt{\epsilon}$, we infer from 3.20 that

$$
\zeta\left(\mathbf{x}_{k}(y)\right)=(-1)^{i} \kappa_{k} a+O(E)
$$

and

$$
\sinh \zeta\left(\mathbf{x}_{k}(y)\right)=(-1)^{i} \kappa_{k} a+O(E) .
$$

Combining (3.36), 3.64) and 3.65), one can see that 3.60), 3.61) and 3.62 follow from (3.37), 3.38), and 3.39), respectively.

Then, using (2.48) and the definitions $(3.12)$ of $m_{1}$ and $m_{2}$, we immediately obtain the following corollary. 
Corollary 3.9. For $|y|<L_{0}$, we have

$$
\begin{aligned}
& q_{11}\left(\mathbf{x}_{2}(y)\right)-q_{11}\left(\mathbf{x}_{1}(y)\right)=m_{1}^{-1} \sqrt{\epsilon}+O(E), \\
& q_{12}\left(\mathbf{x}_{2}(y)\right)-q_{12}\left(\mathbf{x}_{1}(y)\right)=q_{21}\left(\mathbf{x}_{2}(y)\right)-q_{21}\left(\mathbf{x}_{1}(y)\right)=O(\sqrt{\epsilon} \tau|y|), \\
& q_{22}\left(\mathbf{x}_{2}(y)\right)-q_{22}\left(\mathbf{x}_{1}(y)\right)=m_{2}^{-1} \sqrt{\epsilon}+O(E),
\end{aligned}
$$

where $E$ is given by (3.63).

We then obtain the following lemma for estimates of the derivatives of $\mathbf{q}_{j}$.

Lemma 3.10. Let $\mathbf{x}_{k}(y)$ be the defining functions for $\partial D_{k}$ for $k=1,2$ as given in (2.52). For $|y|<L_{0}$, the following holds:

$$
\begin{aligned}
\left|\frac{d}{d y} \mathbf{q}_{j}\left(\mathbf{x}_{k}(y)\right)\right| & \lesssim \sqrt{\epsilon} \\
\left|\frac{d^{2}}{d y^{2}} q_{11}\left(\mathbf{x}_{k}(y)\right)\right|+\left|\frac{d^{2}}{d y^{2}} q_{22}\left(\mathbf{x}_{k}(y)\right)\right| & \lesssim \frac{\sqrt{\epsilon}}{\epsilon+y^{2}}, \\
\left|\frac{d^{2}}{d y^{2}} q_{12}\left(\mathbf{x}_{k}(y)\right)\right| & \lesssim \sqrt{\epsilon}
\end{aligned}
$$

Proof. We only prove inequalities corresponding to $\mathbf{q}_{1}\left(\mathbf{x}_{1}(y)\right)$. Those for other cases, namely, $\mathbf{q}_{1}\left(\mathbf{x}_{2}(y)\right)$ and $\mathbf{q}_{2}\left(\mathbf{x}_{k}(y)\right)$, can be treated similarly.

For ease of notation, let us define $\varphi(y)$ and $\Phi(y)$ by

$$
\varphi(y):=\zeta\left(\mathbf{x}_{1}(y)\right), \quad \Phi(y):=A_{\zeta}\left(\mathbf{x}_{1}(y)\right) .
$$

We see from 3.20$)-3.22$ that

$$
|\varphi(y)| \lesssim \sqrt{\epsilon}, \quad\left|\varphi^{\prime}(y)\right| \lesssim \sqrt{\epsilon}, \quad\left|\varphi^{\prime \prime}(y)\right| \lesssim \frac{\sqrt{\epsilon}}{\epsilon+y^{2}}
$$

We also have

$$
|\Phi(y)| \approx 1, \quad\left|\Phi^{\prime}(y)\right| \lesssim|y|, \quad\left|\Phi^{\prime \prime}(y)\right| \lesssim 1 .
$$

The first estimate in the above is an immediate consequence of (3.54), and the last two can be proved using the definition (3.34) of $A_{\zeta}(\mathbf{x})$. In fact, straightforward computations yield

$$
\Phi^{\prime}(y)=-\frac{1}{2 a^{2} \Phi}\left(y^{2} \varphi^{\prime} \sinh 2 \varphi+2 y \sinh ^{2} \varphi\right),
$$

and

$$
\begin{aligned}
\Phi^{\prime \prime}(y)=- & \frac{1}{2 a^{2} \Phi}\left(4 y \varphi^{\prime} \sinh 2 \varphi+y^{2} \varphi^{\prime \prime} \sinh 2 \varphi+2 y^{2}\left(\varphi^{\prime}\right)^{2} \cosh 2 \varphi+2 \sinh ^{2} \varphi\right) \\
& +\frac{\Phi^{\prime}}{2 a^{2} \Phi^{2}}\left(y^{2} \varphi^{\prime} \sinh 2 \varphi+2 y \sinh ^{2} \varphi\right) .
\end{aligned}
$$

Then, using 3.72 and the fact that $|\Phi| \approx 1$, we obtain

$$
\begin{aligned}
&\left|\Phi^{\prime}(y)\right| \lesssim \frac{1}{\epsilon}\left(y^{2} \epsilon+|y| \epsilon\right) \lesssim|y|, \\
&\left|\Phi^{\prime \prime}(y)\right| \lesssim \frac{1}{\epsilon}\left(|y| \epsilon+y^{2} \frac{\sqrt{\epsilon}}{\epsilon+y^{2}} \sqrt{\epsilon}+y^{2} \epsilon+\epsilon\right)+\frac{|y|}{\epsilon}\left(y^{2} \epsilon+|y| \epsilon\right) \lesssim 1 .
\end{aligned}
$$


We have from $(3.37)$ and $(3.38)$ that

$$
\begin{aligned}
& \frac{d}{d y} q_{11}\left(\mathbf{x}_{1}(y)\right)=\alpha_{1} \varphi^{\prime}-\alpha_{2}\left(\Phi \varphi^{\prime} \cosh \varphi+\Phi^{\prime} \sinh \varphi\right), \\
& \frac{d}{d y} q_{12}\left(\mathbf{x}_{1}(y)\right)=\alpha_{2} a^{-1}\left(\sinh ^{2} \varphi+y \varphi^{\prime} \sinh 2 \varphi\right), \\
& \frac{d^{2}}{d y^{2}} q_{11}\left(\mathbf{x}_{1}(y)\right)=\alpha_{1} \varphi^{\prime \prime}-\alpha_{2}\left(\left(\Phi^{\prime} \varphi^{\prime}+\Phi \varphi^{\prime \prime}+\Phi^{\prime} \varphi^{\prime}\right) \cosh \varphi+\left(\Phi \varphi^{\prime 2}+\Phi^{\prime \prime}\right) \sinh \varphi\right), \\
& \frac{d^{2}}{d y^{2}} q_{12}\left(\mathbf{x}_{1}(y)\right)=\alpha_{2} a^{-1}\left(2 \varphi^{\prime} \sinh 2 \varphi+y \varphi^{\prime \prime} \sinh 2 \varphi+2 y \varphi^{\prime 2} \cosh 2 \varphi\right) .
\end{aligned}
$$

Since $a \approx \sqrt{\epsilon},(3.69)-(3.71)$ now follow from 3.72 and $(3.73)$.

We now estimate $\mathbf{q}_{3}$ whose behavior resembles that of the solution $\mathbf{h}_{3}=\left(h_{31}, h_{32}\right)^{T}$ to 3.1) for $j=3$. Since $\left.\mathbf{h}_{3}\right|_{\partial D_{i}}=\frac{(-1)^{i}}{2}(-y, x)^{T}$ for $i=1,2$, we see that

$$
\mathbf{h}_{3}\left(\mathbf{x}_{2}(y)\right)-\mathbf{h}_{3}\left(\mathbf{x}_{1}(y)\right)=\left(-y, \frac{f_{1}(y)+f_{2}(y)}{2}\right) .
$$

Since $\left|\mathbf{x}_{2}(y)-\mathbf{x}_{1}(y)\right|=f_{1}(y)+f_{2}(y)$, one can expect that the following holds for small $\epsilon>0$ and for $(x, y)$ near the origin:

$$
\partial_{1} h_{31} \approx \frac{-y}{f_{1}(y)+f_{2}(y)}+O(1)=-\frac{y}{\epsilon+\frac{1}{2}\left(\kappa_{1}+\kappa_{2}\right) y^{2}}+O(1)
$$

and

$$
\left|\partial_{2} h_{31}(x, y)\right|+\left|\partial_{1} h_{32}(x, y)\right|+\left|\partial_{2} h_{32}(x, y)\right| \lesssim 1 .
$$

The following lemma shows that $\mathbf{q}_{3}$ has the exactly same local behavior.

Lemma 3.11. For $\mathbf{x}=(x, y) \in \Pi_{L}$, we have

$$
\partial_{1} q_{31}(\mathbf{x})=-\frac{y}{\epsilon+\frac{1}{2}\left(\kappa_{1}+\kappa_{2}\right) y^{2}}+O(1)
$$

and

$$
\left|\partial_{2} q_{31}(\mathbf{x})\right|+\left|\partial_{1} q_{32}(\mathbf{x})\right|+\left|\partial_{2} q_{32}(\mathbf{x})\right| \lesssim 1 \text {. }
$$

Proof. Let $m_{3}$ be the number defined by $(3.16)$. For ease of computation, we decompose $\mathbf{q}_{3}$ as $\mathbf{q}_{3}=\widetilde{\mathbf{q}}_{3}+\mathbf{w}$ where $\mathbf{w}(\mathbf{x})=-m_{3}\left(\boldsymbol{\Gamma}^{\perp}\left(\mathbf{x}-\mathbf{c}_{1}\right)+\boldsymbol{\Gamma}^{\perp}\left(\mathbf{x}-\mathbf{c}_{2}\right)\right)$. It is clear that $|\nabla \mathbf{w}(\mathbf{x})| \lesssim 1$ for $\mathbf{x} \in \Pi_{L}$.

Now we consider $\widetilde{\mathbf{q}}_{3}=\left(\tilde{q}_{31}, \tilde{q}_{32}\right)^{T}$, which is given by

$$
\widetilde{\mathbf{q}}_{3}=m_{3}\left(\boldsymbol{\Gamma}^{\perp}\left(\mathbf{x}-\mathbf{p}_{1}\right)+\boldsymbol{\Gamma}^{\perp}\left(\mathbf{x}-\mathbf{p}_{2}\right)\right)+m_{3} \alpha_{2} a\left(\frac{\left(\mathbf{x}-\mathbf{p}_{1}\right)^{\perp}}{\left|\mathbf{x}-\mathbf{p}_{1}\right|^{2}}-\frac{\left(\mathbf{x}-\mathbf{p}_{2}\right)^{\perp}}{\left|\mathbf{x}-\mathbf{p}_{2}\right|^{2}}\right) .
$$


From the definition 3.13 of $\boldsymbol{\Gamma}^{\perp}$, we have

$$
\begin{aligned}
\tilde{q}_{31}(\mathbf{x})= & m_{3} \alpha_{1} \sum_{i=1}^{2} \arg \left(x-(-1)^{i} a+i y\right) \\
& -m_{3} \alpha_{2} \sum_{i=1}^{2} \frac{\left(x-(-1)^{i} a\right)(-y)}{\left(x-(-1)^{i} a\right)^{2}+y^{2}}+m_{3} \alpha_{2} a \sum_{i=1}^{2} \frac{(-1)^{i+1}(-y)}{\left(x-(-1)^{i} a\right)^{2}+y^{2}}, \\
\tilde{q}_{32}(\mathbf{x})= & -m_{3} \alpha_{2} \sum_{i=1}^{2} \frac{\left(x-(-1)^{i} a\right)^{2}}{\left(x-(-1)^{i} a\right)^{2}+y^{2}}+m_{3} \alpha_{2} a \sum_{i=1}^{2} \frac{(-1)^{i+1}\left(x-(-1)^{i} a\right)}{\left(x-(-1)^{i} a\right)^{2}+y^{2}} .
\end{aligned}
$$

Straightforward computations yield

$$
\begin{aligned}
& \partial_{1} \tilde{q}_{31}(\mathbf{x})=-\left(\kappa_{1}+\kappa_{2}\right)^{-1} f(\mathbf{x})-2 m_{3} \alpha_{2} x y\left[x h_{+}(\mathbf{x})+a h_{-}(\mathbf{x})\right], \\
& \partial_{2} \tilde{q}_{31}(\mathbf{x})=m_{3}\left(\alpha_{1}+\alpha_{2}\right) x g_{+}(\mathbf{x})+m_{3} \alpha_{1} a g_{-}(\mathbf{x})-2 m_{3} \alpha_{2} x y^{2} h_{+}(\mathbf{x}) \text {, } \\
& \partial_{1} \tilde{q}_{32}(\mathbf{x})=-2 m_{3} \alpha_{2} x g_{+}(\mathbf{x})-m_{3} \alpha_{2} a g_{-}(\mathbf{x}) \\
& +2 m_{3} \alpha_{2} x\left[\left(x^{2}+a^{2}\right) h_{+}(\mathbf{x})+2 a x h_{-}(\mathbf{x})\right], \\
& \partial_{2} \tilde{q}_{32}(\mathbf{x})=2 m_{3} \alpha_{2} x y\left[x h_{+}(\mathbf{x})+a h_{-}(\mathbf{x})\right] \text {, }
\end{aligned}
$$

where $f, g_{ \pm}$and $h_{ \pm}$are defined by

$$
\begin{aligned}
f(\mathbf{x}) & =\frac{y}{(x+a)^{2}+y^{2}}+\frac{y}{(x-a)^{2}+y^{2}}, \\
g_{ \pm}(\mathbf{x}) & =\frac{1}{(x+a)^{2}+y^{2}} \pm \frac{1}{(x-a)^{2}+y^{2}} \\
h_{ \pm}(\mathbf{x}) & =\frac{1}{\left((x+a)^{2}+y^{2}\right)^{2}} \pm \frac{1}{\left((x-a)^{2}+y^{2}\right)^{2}} .
\end{aligned}
$$

Since $a \approx \sqrt{\epsilon},|x| \lesssim \epsilon+y^{2}$ and $(x \pm a)+y^{2} \approx \epsilon+y^{2}$, we see that

$$
\begin{aligned}
\left|g_{+}(\mathbf{x})\right| \lesssim \frac{1}{\epsilon+y^{2}}, \quad\left|h_{+}(\mathbf{x})\right| \lesssim \frac{1}{\left(\epsilon+y^{2}\right)^{2}}, \\
\left|g_{-}(\mathbf{x})\right|=\left|\frac{4 a x}{\left((x+a)^{2}+y^{2}\right)\left((x-a)^{2}+y^{2}\right)}\right| \lesssim \frac{\sqrt{\epsilon}}{\epsilon+y^{2}}, \\
\left|h_{-}(\mathbf{x})\right|=\left|\frac{4 a x\left((x+a)^{2}+(x-a)^{2}+2 y^{2}\right)}{\left((x+a)^{2}+y^{2}\right)^{2}\left((x-a)^{2}+y^{2}\right)^{2}}\right| \lesssim \frac{\sqrt{\epsilon}}{\left(\epsilon+y^{2}\right)^{2}} .
\end{aligned}
$$

Therefore, we obtain

$$
\begin{aligned}
& \left|\partial_{1} \tilde{q}_{31}(\mathbf{x})+\left(\kappa_{1}+\kappa_{2}\right)^{-1} f(\mathbf{x})\right| \lesssim \frac{\left(\epsilon+y^{2}\right)^{2} y}{\left(\epsilon+y^{2}\right)^{2}}+\frac{\epsilon\left(\epsilon+y^{2}\right) y}{\left(\epsilon+y^{2}\right)^{2}} \lesssim 1 \\
& \left|\partial_{2} \tilde{q}_{31}(\mathbf{x})\right| \lesssim \frac{\epsilon+y^{2}}{\epsilon+y^{2}}+\sqrt{\epsilon} \frac{\sqrt{\epsilon}}{\epsilon+y^{2}}++\frac{\left(\epsilon+y^{2}\right) y^{2}}{\left(\epsilon+y^{2}\right)^{2}} \lesssim 1 \\
& \left|\partial_{1} \tilde{q}_{32}(\mathbf{x})\right| \lesssim \frac{\epsilon+y^{2}}{\epsilon+y^{2}}+\sqrt{\epsilon} \frac{\sqrt{\epsilon}}{\epsilon+y^{2}}+\frac{\left(\epsilon+y^{2}\right)^{3}+\epsilon\left(\epsilon+y^{2}\right)}{\left(\epsilon+y^{2}\right)^{2}}+\frac{\epsilon\left(\epsilon+y^{2}\right)}{\left(\epsilon+y^{2}\right)^{2}} \lesssim 1 \\
& \left|\partial_{2} \tilde{q}_{32}(\mathbf{x})\right| \lesssim \frac{\left(\epsilon+y^{2}\right)^{2} y}{\left(\epsilon+y^{2}\right)^{2}}+\frac{\epsilon\left(\epsilon+y^{2}\right) y}{\left(\epsilon+y^{2}\right)^{2}} \lesssim 1
\end{aligned}
$$


Now it remains to show that

$$
f(\mathbf{x})=\frac{\left(\kappa_{1}+\kappa_{2}\right) y}{\epsilon+\frac{1}{2}\left(\kappa_{1}+\kappa_{2}\right) y^{2}}+O(1) .
$$

Since

$$
\begin{aligned}
\left|\frac{y}{(x \pm a)^{2}+y^{2}}-\frac{y}{a^{2}+y^{2}}\right| & \lesssim\left|\frac{y\left(x^{2} \pm 2 a x\right)}{\left((x \pm a)^{2}+y^{2}\right)\left(a^{2}+y^{2}\right)}\right| \\
& \lesssim \frac{y\left(\left(\epsilon+y^{2}\right)^{2}+\sqrt{\epsilon}\left(\epsilon+y^{2}\right)\right)}{\left(\epsilon+y^{2}\right)^{2}} \lesssim 1
\end{aligned}
$$

we see that

$$
f(\mathbf{x})=\frac{2 y}{a^{2}+y^{2}}+O(1) .
$$

Since

$$
\frac{y}{a^{2}+y^{2}}=\frac{y}{2 \epsilon /\left(\kappa_{1}+\kappa_{2}\right)+O\left(\epsilon^{2}\right)+y^{2}}=\frac{\frac{1}{2}\left(\kappa_{1}+\kappa_{2}\right) y}{\epsilon+\frac{1}{2}\left(\kappa_{1}+\kappa_{2}\right) y^{2}}+O(1),
$$

the desired estimate 3.78 follows. This completes the proof.

Lemma 3.12. The following holds:

$$
\left|\nabla \mathbf{q}_{3}(0,0)\right| \lesssim 1, \quad \nabla \mathbf{q}_{3}(0, a)=\frac{\sqrt{\kappa_{1}+\kappa_{2}}}{\sqrt{2 \epsilon}} \mathbf{e}_{1} \otimes \mathbf{e}_{1}+O(1) .
$$

Moreover, we have

$$
\left\|\nabla \mathbf{q}_{3}\right\|_{L^{\infty}\left(D^{e} \backslash \Pi_{L}\right)} \lesssim 1
$$

and

$$
\left\|\nabla \mathbf{q}_{3}\right\|_{L^{\infty}\left(D^{e}\right)} \approx \frac{1}{\sqrt{\epsilon}} .
$$

Proof. The estimates in $(3.79$ are consequences of Lemma 3.11. The estimate (3.81) is a consequence of Lemma 3.11 and 3.80 .

To prove 3.80 , recall that

$$
\begin{aligned}
\mathbf{q}_{3}(\mathbf{x})= & m_{3}\left(\boldsymbol{\Gamma}^{\perp}\left(\mathbf{x}-\mathbf{p}_{1}\right)-\boldsymbol{\Gamma}^{\perp}\left(\mathbf{x}-\mathbf{c}_{1}\right)\right) \mathbf{e}_{1}+m_{3}\left(\boldsymbol{\Gamma}^{\perp}\left(\mathbf{x}-\mathbf{p}_{2}\right)-\boldsymbol{\Gamma}^{\perp}\left(\mathbf{x}-\mathbf{c}_{2}\right)\right) \mathbf{e}_{1} \\
& -m_{3} \alpha_{2} a\left(\frac{\left(\mathbf{x}-\mathbf{p}_{1}\right)^{\perp}}{\left|\mathbf{x}-\mathbf{p}_{1}\right|^{2}}-\frac{\left(\mathbf{x}-\mathbf{p}_{2}\right)^{\perp}}{\left|\mathbf{x}-\mathbf{p}_{2}\right|^{2}}\right) .
\end{aligned}
$$

If $\mathbf{x} \in D^{e} \backslash \Pi_{L}$, then $1 \lesssim|\mathbf{x}-\mathbf{c}|$ for all $\mathbf{c}$ on the line segment $\overline{\mathbf{c}_{1} \mathbf{c}_{2}}$. Note that $\mathbf{p}_{1}$ and $\mathbf{p}_{2}$ are on $\overline{\mathbf{c}_{1} \mathbf{c}_{2}}$. So, all the terms in parentheses above and their gradients are bounded. So, (3.80) follows.

\subsection{Approximations by singular functions}

In this section we prove (3.11). More precisely, we prove the following proposition. 
Proposition 3.13. For $j=1,2$, let $\mathbf{h}_{j}$ be the solution to (3.1) in $\mathcal{A}^{*}$ and $m_{j}$ be the constant defined in (3.12). Then it holds that

$$
\mathbf{h}_{j}=\frac{m_{j}}{\sqrt{\epsilon}} \mathbf{q}_{j}+\mathbf{r}_{j}
$$

where $\nabla \mathbf{r}_{j}$ satisfies

$$
\int_{D^{e}} \mathbb{C} \widehat{\nabla} \mathbf{r}_{j}: \widehat{\nabla} \mathbf{r}_{j} \lesssim 1
$$

To prove Proposition 3.13 we apply the variational principle. We emphasize that this is possible only because the singular function $\mathbf{q}_{j}$ is the solution to the Lamé system, namely, $\mathcal{L}_{\lambda, \mu} \mathbf{q}_{j}=0$ in $D^{e}$, and so is $\mathbf{r}_{j}$. Note that $\mathbf{r}_{j} \in \mathcal{A}^{*}$ and

$$
\mathbf{r}_{j}=(-1)^{i} \frac{1}{2} \Psi_{j}-\frac{m_{j}}{\sqrt{\epsilon}} \mathbf{q}_{j} \quad \text { on } \partial D_{i}, \quad i=1,2 .
$$

Let

$$
W_{j}=\left\{\mathbf{v} \in \mathcal{A}^{*}|\mathbf{v}|_{\partial D_{i}}=(-1)^{i} \frac{1}{2} \Psi_{j}-\frac{m_{j}}{\sqrt{\epsilon}} \mathbf{q}_{j}\right\},
$$

and let $\mathcal{E}_{D^{e}}$ be the energy functional defined in (2.36). By the variational principle (2.37), we have

$$
\mathcal{E}_{D^{e}}\left[\mathbf{r}_{j}\right]=\min _{\mathbf{v} \in W_{j}} \mathcal{E}_{D^{e}}[\mathbf{v}] .
$$

We define the test function $\mathbf{r}_{j}^{K}$ as follows: for $(x, y) \in \Pi_{L_{0}}$ let

$$
\mathbf{r}_{j}^{K}(x, y):=\frac{\mathbf{r}_{j}\left(\mathbf{x}_{2}(y)\right)-\mathbf{r}_{j}\left(\mathbf{x}_{1}(y)\right)}{f_{1}(y)+f_{2}(y)}\left[x+f_{1}(y)\right]+\mathbf{r}_{j}\left(\mathbf{x}_{1}(y)\right) .
$$

Note that

$$
\mathbf{r}_{j}^{K}=(-1)^{i} \frac{1}{2} \Psi_{j}-\frac{m_{j}}{\sqrt{\epsilon}} \mathbf{q}_{j}=\mathbf{r}_{j} \quad \text { on } \partial D_{i} \cap \partial \Pi_{L_{0}}, \quad i=1,2,
$$

and $\mathbf{r}_{j}^{K}$ is a linear interpolation of $\left.\mathbf{r}_{j}\right|_{\partial D^{e}}$ in the $x$-direction. So, in $\Pi_{L_{0}}, \mathbf{r}_{j}^{K}(x, y)$ is a linear function of $x$ for each fixed $y$. Let $B_{0}$ be a disk containing $\overline{D_{1} \cup D_{2}}$, and extend $\mathbf{r}_{j}^{K}$ to $D^{e} \backslash \Pi_{L_{0}}$ so that $\left.\mathbf{r}_{j}^{K}\right|_{\mathbb{R}^{2} \backslash B_{0}}=0,\left\|\mathbf{r}_{j}^{K}\right\|_{H^{1}\left(D^{e} \backslash \Pi_{L_{0}}\right)} \lesssim 1$, and the boundary condition (3.87) holds on $\partial D_{i}$ for $i=1,2$. Then, $\mathbf{r}_{j}^{K}$ belongs to $W_{j}$.

We have the following lemma.

Lemma 3.14. We have, for $(x, y) \in \Pi_{L_{0}}$,

$$
\left|\nabla \mathbf{r}_{j}^{K}(x, y)\right| \lesssim 1+\frac{\tau|y|}{\epsilon+y^{2}}
$$

Proof. We prove $(3.88)$ for $j=1$. The case for $j=2$ can be proved in a similar way.

Let us write $\mathbf{r}_{j}^{K}(\mathbf{x})=\left(r_{j 1}^{K}(\mathbf{x}), r_{j 2}^{K}(\mathbf{x})\right)^{T}$. To keep the expressions simple, we introduce

$$
\begin{aligned}
& d(y):=f_{1}(y)+f_{2}(y), \\
& \phi(y):=1-\frac{m_{1}}{\sqrt{\epsilon}}\left[q_{11}\left(\mathbf{x}_{2}(y)\right)-q_{11}\left(\mathbf{x}_{1}(y)\right)\right], \\
& \eta(y):=-\frac{1}{2}-\frac{m_{1}}{\sqrt{\epsilon}} q_{11}\left(\mathbf{x}_{1}(y)\right) .
\end{aligned}
$$


Then $r_{11}^{K}$ can be rewritten as

$$
r_{11}^{K}(x, y)=\frac{\phi(y)}{d(y)} x+\frac{\phi(y) f_{1}(y)}{d(y)}+\eta(y), \quad(x, y) \in \Pi_{L_{0}} .
$$

Straightforward computations show that

$$
\begin{aligned}
& \partial_{1} r_{11}^{K}=\frac{\phi}{d}, \\
& \partial_{2} r_{11}^{K}=\left[\frac{\phi^{\prime}}{d}-\frac{\phi d^{\prime}}{d^{2}}\right] x+\frac{\phi^{\prime} f_{1}}{d}+\frac{\phi f_{1}^{\prime}}{d}-\frac{\phi f_{1} d^{\prime}}{d^{2}}+\eta^{\prime} .
\end{aligned}
$$

Note that

$$
d(y) \approx \epsilon+y^{2}, \quad\left|d^{\prime}(y)\right| \lesssim|y|, \quad\left|d^{\prime \prime}(y)\right| \lesssim 1 .
$$

Note also that, from 3.12 , Corollary 3.9 and Lemma 3.10 , we have

$$
|\phi(y)| \lesssim \epsilon+y^{2}+\tau|y|, \quad\left|\phi^{\prime}(y)\right|,\left|\eta^{\prime}(y)\right| \lesssim 1
$$

From $3.90-3.93$ and the fact that $|x| \lesssim \epsilon+y^{2}$ for $(x, y) \in \Pi_{L}$, we have

$$
\begin{aligned}
\left|\partial_{1} r_{11}^{K}\right| \lesssim & \frac{\epsilon+y^{2}+\tau|y|}{\epsilon+y^{2}} \lesssim 1+\frac{\tau|y|}{\epsilon+y^{2}}, \\
\left|\partial_{2} r_{11}^{K}\right| \lesssim & {\left[\frac{1}{\epsilon+y^{2}}+\frac{(\epsilon+|y|)|y|}{\left(\epsilon+y^{2}\right)^{2}}\right]\left(\epsilon+y^{2}\right) } \\
& +\frac{\epsilon+y^{2}}{\epsilon+y^{2}}+\frac{(\epsilon+|y|)|y|}{\epsilon+y^{2}}+\frac{(\epsilon+|y|)\left(\epsilon+y^{2}\right)|y|}{\left(\epsilon+y^{2}\right)^{2}}+1 \lesssim 1 .
\end{aligned}
$$

In a similar way, one can see that

$$
\left|\partial_{1} r_{12}^{K}\right| \lesssim 1+\frac{\tau|y|}{\epsilon+y^{2}}, \quad\left|\partial_{2} r_{12}^{K}\right| \lesssim 1
$$

This completes the proof.

Proof of Proposition 3.13. By the variational principle (3.85), we have

$$
\mathcal{E}_{D^{e}}\left[\mathbf{r}_{j}\right] \leq \mathcal{E}_{D^{e}}\left[\mathbf{r}_{j}^{K}\right] \lesssim\left\|\nabla \mathbf{r}_{j}^{K}\right\|_{L^{2}\left(D^{e}\right)}^{2} .
$$

It follows from Lemma 3.14 that

$$
\begin{aligned}
\int_{D^{e}}\left|\nabla \mathbf{r}_{j}^{K}\right|^{2} & \lesssim \int_{\Pi_{L_{0}}}\left|\nabla \mathbf{r}_{j}^{K}\right|^{2}+\int_{D^{e} \backslash \Pi_{L_{0}}}\left|\nabla \mathbf{r}_{j}^{K}\right|^{2} \\
& \lesssim \int_{-L_{0}}^{L_{0}} \int_{-f_{1}(y)}^{f_{2}(y)}\left(\frac{\epsilon+|y|}{\epsilon+y^{2}}\right)^{2} d x d y+1 \\
& \lesssim \int_{-L_{0}}^{L_{0}} \frac{(\epsilon+|y|)^{2}}{\epsilon+y^{2}} d y+1 \lesssim 1 .
\end{aligned}
$$

So the proof is complete. 


\section{Stress concentration-boundary value problem}

This section deals with the stress concentration, i.e., the gradient blow-up of the solution to the boundary value problem (2.6). We characterize the stress concentration in the narrow region between two inclusions in terms of the singular functions $\mathbf{q}_{j}$ defined in (3.8), (3.9), and (3.15). The main results (Theorem 4.6 and 4.7) are stated and proved in subsection 4.1. Preliminary results required for proving main ones are also stated in the same subsection. Their proofs are given in subsequent subsections. At the end of subsection 4.1 we include a brief comparison of this paper's method with that of [9] where the upper bound of the gradient blow-up is obtained.

\subsection{Characterization of stress concentration-BVP}

We first introduce functions $\mathbf{h}_{\Omega, j}$ for the boundary value problem, analogously to the functions $\mathbf{h}_{j}$ defined in (3.1) for the free space problem. They are solutions to the following problem:

$$
\begin{cases}\mathcal{L}_{\lambda, \mu} \mathbf{h}_{\Omega, j}=0 & \text { in } \widetilde{\Omega}, \\ \mathbf{h}_{\Omega, j}=\frac{(-1)^{i}}{2} \Psi_{j} & \text { on } \partial D_{i}, i=1,2, \\ \mathbf{h}_{\Omega, j}=0 & \text { on } \partial \Omega .\end{cases}
$$

One can easily see that the solution $\mathbf{u}$ to $(2.6)$ admits the decomposition

$$
\mathbf{u}=\mathbf{v}_{\Omega}-\sum_{j=1}^{3}\left(c_{1 j}-c_{2 j}\right) \mathbf{h}_{\Omega, j} \quad \text { in } \widetilde{\Omega},
$$

where $\mathbf{v}_{\Omega}$ is the solution to $\mathcal{L}_{\lambda, \mu} \mathbf{v}_{\Omega}=0$ in $\widetilde{\Omega}$ with the boundary condition

$$
\mathbf{v}_{\Omega}=\frac{1}{2} \sum_{j=1}^{3}\left(c_{1 j}+c_{2 j}\right) \Psi_{j} \quad \text { on } \partial D_{1} \cup \partial D_{2} .
$$

Note that

$$
\left.\mathbf{v}_{\Omega}\right|_{\partial D_{1}}-\left.\mathbf{v}_{\Omega}\right|_{\partial D_{2}}=\frac{1}{2}\left(c_{13}+c_{23}\right)\left(\left.\Psi_{3}\right|_{\partial D_{1}}-\left.\Psi_{3}\right|_{\partial D_{2}}\right)=O(|\mathbf{x}|)
$$

from which one expects that $\nabla \mathbf{v}_{\Omega}$ does not blow up even when $\epsilon \rightarrow 0$. In fact, it was proved in [9] that

$$
\left\|\nabla \mathbf{v}_{\Omega}\right\|_{L^{\infty}(\widetilde{\Omega})} \lesssim\|\mathbf{g}\|_{C^{1, \gamma}(\partial \Omega)}
$$

So the singular behavior of $\nabla \mathbf{u}$ is determined by the function $\sum_{j=1}^{3}\left(c_{1 j}-c_{2 j}\right) \mathbf{h}_{\Omega, j}$.

In the sequel, we investigate asymptotic behavior of $c_{1 j}-c_{2 j}$ and $\mathbf{h}_{\Omega, j}$ as $\epsilon \rightarrow 0$. For doing so, we introduce the following boundary integrals:

$$
\mathcal{I}_{j k}:=\int_{D^{e}} \mathbb{C} \widehat{\nabla} \mathbf{h}_{j}: \widehat{\nabla} \mathbf{h}_{k} \quad \text { and } \quad \mathcal{J}_{\Omega, k}:=\left.\int_{\partial D^{e}} \frac{\partial \mathbf{h}_{k}}{\partial \nu}\right|_{+} \cdot \mathbf{H}_{\Omega}, \quad j, k=1,2,3,
$$

where $\mathbf{h}_{j}$ is the solution to (3.1) in $\mathcal{A}^{*}$ and $\mathbf{H}_{\Omega}$ is the function defined by (2.17). We emphasize that $\mathcal{I}_{j k}$ is defined by $\mathbf{h}_{j}$, not by $\mathbf{h}_{\Omega, j}$.

The relation among $c_{1 j}-c_{2 j}, \mathcal{I}_{j k}$ and $\mathcal{J}_{\Omega, k}$ is given by the following lemma. 
Lemma 4.1. The constants $c_{i j}$ appearing in (2.6) satisfy

$$
\left[\begin{array}{lll}
\mathcal{I}_{11} & \mathcal{I}_{12} & \mathcal{I}_{13} \\
\mathcal{I}_{12} & \mathcal{I}_{22} & \mathcal{I}_{23} \\
\mathcal{I}_{13} & \mathcal{I}_{23} & \mathcal{I}_{33}
\end{array}\right]\left[\begin{array}{l}
c_{11}-c_{21} \\
c_{12}-c_{22} \\
c_{13}-c_{23}
\end{array}\right]=\left[\begin{array}{l}
\mathcal{J}_{\Omega, 1} \\
\mathcal{J}_{\Omega, 2} \\
\mathcal{J}_{\Omega, 3}
\end{array}\right] .
$$

By inverting (4.6), we will see that the asymptotic behavior of $c_{1 j}-c_{2 j}$ as $\epsilon \rightarrow 0$ can be described in terms of $\mathcal{K}_{\Omega, j}$ which are defined by

$$
\mathcal{K}_{\Omega, 1}=\mathcal{J}_{\Omega, 1}-\frac{\mathcal{J}_{\Omega, 3} \mathcal{I}_{13}}{\mathcal{I}_{33}}, \quad \mathcal{K}_{\Omega, 2}=\mathcal{J}_{\Omega, 2}-\frac{\mathcal{J}_{\Omega, 3} \mathcal{I}_{23}}{\mathcal{I}_{33}}, \quad \mathcal{K}_{\Omega, 3}=\frac{\mathcal{J}_{\Omega, 3}}{\mathcal{I}_{33}} .
$$

In fact, the following propositions hold. Here, we mention that they are consequences of Proposition 3.13 , which is proved by the variational principle and the properties of singular functions $\mathbf{q}_{j}$.

Proposition 4.2. For $j=1,2,3$, we have

$$
\left|\mathcal{K}_{\Omega, j}\right| \lesssim\|\mathbf{g}\|_{C^{1, \gamma}(\partial \Omega)}
$$

Proposition 4.3. We have

$$
\begin{aligned}
& c_{11}-c_{21}=\mathcal{K}_{\Omega, 1} m_{1}^{-1} \sqrt{\epsilon}+O(\sqrt{\epsilon} \widetilde{E}), \\
& c_{12}-c_{22}=\mathcal{K}_{\Omega, 2} m_{2}^{-1} \sqrt{\epsilon}+O(\sqrt{\epsilon} \widetilde{E}), \\
& c_{13}-c_{23}=\mathcal{K}_{\Omega, 3}+O(\widetilde{E}),
\end{aligned}
$$

where

$$
\widetilde{E}:=(\sqrt{\epsilon}+\tau \sqrt{\epsilon}|\ln \epsilon|)\|\mathbf{g}\|_{C^{1, \gamma}(\partial \Omega)} .
$$

As an immediate consequence of Propositions 4.2 and 4.3 , we obtain the following corollary.

Corollary 4.4. We have

$$
\left|c_{11}-c_{21}\right|+\left|c_{12}-c_{22}\right| \lesssim \sqrt{\epsilon}\|\mathbf{g}\|_{C^{1, \gamma}(\partial \Omega)}
$$

and

$$
\left|c_{13}-c_{23}\right| \lesssim\|\mathbf{g}\|_{C^{1, \gamma}(\partial \Omega)}
$$

Regarding the asymptotic behavior of $\mathbf{h}_{\Omega, j}$, we obtain the following proposition.

Proposition 4.5. Let $m_{j}, j=1,2$, be the constant defined by (3.12). We have for $j=1,2$

$$
\mathbf{h}_{\Omega, j}=\frac{m_{j}}{\sqrt{\epsilon}} \mathbf{q}_{j}+\mathbf{r}_{\Omega, j},
$$

where $\mathbf{r}_{\Omega, j}$ satisfies

$$
\begin{cases}\left|\nabla \mathbf{r}_{\Omega, j}(\mathbf{x})\right| \lesssim 1+\frac{\tau|y|}{\epsilon+y^{2}} & \text { for } \mathbf{x} \in \Pi_{L_{0}} \\ \left|\nabla \mathbf{r}_{\Omega, j}(\mathbf{x})\right| \lesssim 1 & \text { for } \mathbf{x} \in \widetilde{\Omega} \backslash \Pi_{L_{0}}\end{cases}
$$


Here, $\tau$ is the constant defined by (2.54). We also have

$$
\mathbf{h}_{\Omega, 3}=\mathbf{q}_{3}+\mathbf{r}_{\Omega, 3} \text { in } \widetilde{\Omega},
$$

where $\mathbf{r}_{\Omega, 3}$ satisfies

$$
\left|\nabla \mathbf{r}_{\Omega, 3}\right| \lesssim 1 \quad \text { in } \widetilde{\Omega} .
$$

It is worth emphasizing that if two inclusions are symmetric with respect to both $x$ and $y$-axes, then $\tau=0$. So we have $\left|\nabla \mathbf{r}_{\Omega, j}\right| \lesssim 1$ in $\widetilde{\Omega}$ for $j=1,2$ as well.

With help of preliminary results presented above, we are now able to state and prove the main results of this section.

Theorem 4.6. Let $\mathbf{u}$ be the solution to (2.6) for some $\mathbf{g} \in C^{1, \gamma}(\partial \Omega)$. The following decomposition holds

$$
\mathbf{u}(\mathbf{x})=\mathbf{b}_{\Omega}(\mathbf{x})-\sum_{j=1}^{3}\left(\mathcal{K}_{\Omega, j}+s_{\Omega, j}\right) \mathbf{q}_{j}(\mathbf{x}), \quad \mathbf{x} \in \widetilde{\Omega},
$$

where $\mathcal{K}_{\Omega, j}$ are the constants defined by (4.7) (so satisfies (4.8)), s, $s_{\Omega, j}$ are constants satisfying

$$
\left|s_{\Omega, j}\right| \lesssim \tau \sqrt{\epsilon}|\ln \epsilon|\|\mathbf{g}\|_{C^{1, \gamma}(\partial \Omega)},
$$

and the function $\mathbf{b}_{\Omega}$ satisfies

$$
\left\|\nabla \mathbf{b}_{\Omega}\right\|_{L^{\infty}(\widetilde{\Omega})} \lesssim\|\mathbf{g}\|_{C^{1, \gamma}(\partial \Omega)}
$$

Theorem 4.7. It holds that

$$
\frac{\sum_{j=1}^{2}\left|\mathcal{K}_{\Omega, j}\right|}{\sqrt{\epsilon}} \lesssim\|\nabla \mathbf{u}\|_{L^{\infty}(\widetilde{\Omega})} \lesssim \frac{\|\mathbf{g}\|_{C^{1, \gamma}(\partial \Omega)}}{\sqrt{\epsilon}} .
$$

The upper estimate in 4.22 was proved in [9]. The lower estimate shows that $\epsilon^{-1 / 2}$ is also the lower bound on the blow-up rate of $\nabla \mathbf{u}$ as $\epsilon \rightarrow 0$, provided that

$$
1 \lesssim \sum_{j=1}^{2}\left|\mathcal{K}_{\Omega, j}\right|
$$

We will show in some special cases that this is the case (see section 6).

Proof of Theorem 4.6. According to Proposition 4.3. $c_{1 j}-c_{2 j}$ can be written as

$$
\begin{aligned}
& c_{11}-c_{21}=\mathcal{K}_{\Omega, 1} m_{1}^{-1} \sqrt{\epsilon}+m_{1}^{-1} \sqrt{\epsilon}\left(s_{\Omega, 1}+s_{\Omega, 1}^{\prime}\right), \\
& c_{12}-c_{22}=\mathcal{K}_{\Omega, 2} m_{2}^{-1} \sqrt{\epsilon}+m_{2}^{-1} \sqrt{\epsilon}\left(s_{\Omega, 2}+s_{\Omega, 2}^{\prime}\right), \\
& c_{13}-c_{23}=\mathcal{K}_{\Omega, 3}+s_{\Omega, 3}+s_{\Omega, 3}^{\prime},
\end{aligned}
$$

where the constants $s_{\Omega, j}$ and $s_{\Omega, j}^{\prime}$ satisfy

$$
\begin{aligned}
& \left|s_{\Omega, j}\right| \lesssim \tau \sqrt{\epsilon}|\ln \epsilon|\|\mathbf{g}\|_{C^{1, \gamma}(\partial \Omega)}, \\
& \left|s_{\Omega, j}^{\prime}\right| \lesssim \sqrt{\epsilon}\|\mathbf{g}\|_{C^{1, \gamma}(\partial \Omega)}
\end{aligned}
$$


By substituting (4.15) and above three identities into 4.2 , we have

$$
\begin{aligned}
\mathbf{u} & =\mathbf{v}_{\Omega}-\left(\sum_{j=1}^{2}\left(c_{1 j}-c_{2 j}\right)\left(\frac{m_{j}}{\sqrt{\epsilon}} \mathbf{q}_{j}+\mathbf{r}_{\Omega, j}\right)\right)-\left(c_{13}-c_{23}\right)\left(\mathbf{q}_{3}+\mathbf{r}_{\Omega, 3}\right) \\
& =\mathbf{v}_{\Omega}-\sum_{j=1}^{3}\left(\mathcal{K}_{\Omega, j}+s_{\Omega, j}+s_{\Omega, j}^{\prime}\right) \mathbf{q}_{j}-\sum_{j=1}^{3}\left(c_{1 j}-c_{2 j}\right) \mathbf{r}_{\Omega, j} .
\end{aligned}
$$

Let

$$
\mathbf{b}_{\Omega}:=\mathbf{u}+\sum_{j=1}^{3}\left(\mathcal{K}_{\Omega, j}+s_{\Omega, j}\right) \mathbf{q}_{j}
$$

Then, from 4.26), we have

$$
\nabla \mathbf{b}_{\Omega}=\nabla \mathbf{v}_{\Omega}-\sum_{j=1}^{3} s_{\Omega, j}^{\prime} \nabla \mathbf{q}_{j}-\sum_{j=1}^{3}\left(c_{1 j}-c_{2 j}\right) \nabla \mathbf{r}_{\Omega, j}=: I_{1}+I_{2}+I_{3} .
$$

We now prove that $I_{j}$ are bounded. That $\left|I_{1}\right| \lesssim\|\mathbf{g}\|_{C^{1, \gamma}(\partial \Omega)}$ is already mentioned in (4.4). By (3.59) and (3.81), we have

$$
\left\|\nabla \mathbf{q}_{j}\right\|_{L^{\infty}(\widetilde{\Omega})} \lesssim \epsilon^{-1 / 2}, \quad j=1,2,3
$$

So, by 4.25), we have

$$
\left|I_{2}\right| \lesssim\|\mathbf{g}\|_{C^{1, \gamma}(\partial \Omega)}
$$

We have from 4.16) and 4.18 that

$$
\left\|\nabla \mathbf{r}_{\Omega, 1}\right\|_{L^{\infty}(\widetilde{\Omega})}+\left\|\nabla \mathbf{r}_{\Omega, 2}\right\|_{L^{\infty}(\widetilde{\Omega})} \lesssim 1+\tau / \sqrt{\epsilon}
$$

and

$$
\left\|\nabla \mathbf{r}_{\Omega, 3}\right\|_{L^{\infty}(\widetilde{\Omega})} \lesssim 1
$$

Therefore, it follows from Corollary 4.4 that

$$
\begin{aligned}
\left|I_{3}\right| & \leq\left|\sum_{j=1}^{2}\left(c_{1 j}-c_{2 j}\right) \nabla \mathbf{r}_{\Omega, j}\right|+\left|\left(c_{13}-c_{23}\right) \nabla \mathbf{r}_{\Omega, 3}\right| \\
& \lesssim(\sqrt{\epsilon}(1+\tau / \sqrt{\epsilon})+1)\|\mathbf{g}\|_{C^{1, \gamma}(\partial \Omega)} \lesssim\|\mathbf{g}\|_{C^{1, \gamma}(\partial \Omega)} .
\end{aligned}
$$

The proof is complete.

Proof of Theorem 4.7. The upper estimate in 4.22 is a consequence of Proposition 4.2 , Theorem 4.6, and 4.27). To derive the lower estimate, we consider $\nabla \mathbf{u}(0,0)$. It follows from Lemma 3.5. Lemma 3.12 and Theorem 4.6 that

$$
\nabla \mathbf{u}(0,0)=-\frac{\mathcal{K}_{\Omega, 1}}{m_{1} \sqrt{\epsilon}} \mathbf{e}_{1} \otimes \mathbf{e}_{1}-\frac{\mathcal{K}_{\Omega, 2}}{m_{2} \sqrt{\epsilon}} \mathbf{e}_{2} \otimes \mathbf{e}_{1}+O(1+\tau \ln \epsilon) .
$$

So we obtain the lower estimate. 
As mentioned earlier, the upper bound in 4.22 was proved in 9 . So, it is helpful to compare the method of that paper with that of this paper. In fact, some of results obtained in [9] will be used for proofs in this section. There, the solution $\mathbf{u}$ to (2.6) is expressed as follows:

$$
\mathbf{u}=\sum_{i=1}^{2} \sum_{k=1}^{3} c_{i k} \mathbf{v}_{i k}+\mathbf{v}_{3},
$$

where $\mathbf{v}_{i k}$ is the solution to

$$
\begin{cases}\mathcal{L}_{\lambda, \mu} \mathbf{v}_{i k}=0 & \text { in } \widetilde{\Omega} \\ \mathbf{v}_{i k}=\Psi_{k} & \text { on } \partial D_{i}, \\ \mathbf{v}_{i k}=0 & \text { on } \partial D_{j} \cup \partial \Omega, j \neq i\end{cases}
$$

and $\mathbf{v}_{3}$ is the solution to

$$
\begin{cases}\mathcal{L}_{\lambda, \mu} \mathbf{v}_{3}=0 & \text { in } \widetilde{\Omega} \\ \mathbf{v}_{3}=0 & \text { on } \partial D_{1} \cup \partial D_{2} \\ \mathbf{v}_{3}=\mathbf{g} & \text { on } \partial \Omega\end{cases}
$$

Note that

$$
\mathbf{h}_{\Omega, k}=-\frac{1}{2} \mathbf{v}_{1 k}+\frac{1}{2} \mathbf{v}_{2 k}, \quad k=1,2,3 .
$$

The $6 \times 6$ linear system of equations for $c_{i k}$ is derived using (2.7). The linear system is truncated to a $3 \times 3$ one and then the difference $c_{1 j}-c_{2 j}$ is expressed using the following integrals:

$$
\begin{aligned}
a_{j k} & :=\left.\int_{\partial D_{1}} \partial_{\nu} \mathbf{v}_{1 j}\right|_{+} \cdot \Psi_{k}=\int_{\widetilde{\Omega}} \mathbb{C} \widehat{\nabla} \mathbf{v}_{1 j}: \widehat{\nabla} \mathbf{v}_{1 k}, \\
b_{k} & :=\left.\int_{\partial D_{1}} \partial_{\nu} \mathbf{v}_{3}\right|_{+} \cdot \Psi_{k},
\end{aligned}
$$

for $j, k=1,2,3$. Note that the integral $a_{j k}$ is similar to the quantity $\mathcal{I}_{j k}$ of this paper. The difference lies in that $\mathcal{I}_{j k}$ is defined using the free space solution $\mathbf{h}_{j}$.

To investigate asymptotic behavior of $a_{j k}$ and $b_{k}$ as $\epsilon \rightarrow 0$, the function $\mathbf{v}_{i k}$ is approximated by $\mathbf{v}_{i k}^{K}$, which is defined by

$$
\left\{\begin{array}{l}
\mathbf{v}_{1 k}^{K}(x, y)=\frac{-x+f_{2}(y)}{f_{1}(y)+f_{2}(y)} \Psi_{k}, \\
\mathbf{v}_{2 k}^{K}(x, y)=\frac{x+f_{1}(y)}{f_{1}(y)+f_{2}(y)} \Psi_{k},
\end{array} \quad(x, y) \in \Pi_{L}, \quad k=1,2,3 .\right.
$$

In fact, it is proved that

$$
\begin{aligned}
& \nabla \mathbf{v}_{i k}(x, y)=\nabla \mathbf{v}_{i k}^{K}(x, y)+O\left(1+\frac{y}{\epsilon+y^{2}}\right) \quad \text { for } k=1,2, \\
& \nabla \mathbf{v}_{i 3}(x, y)=\nabla \mathbf{v}_{i 3}^{K}(x, y)+O(1) .
\end{aligned}
$$

From these approximations which are derived using a new iteration technique, the upper bound on the blow-up rate, $\epsilon^{-1 / 2}$, of $|\nabla \mathbf{u}|$ is obtained in [9]. However, a lower bound has 
not been obtained. It is partly because the functions $\mathbf{v}_{i k}^{K}$ are not solutions of the Lamé system.

In this paper, we introduce new singular functions $\mathbf{q}_{j}$, which are solutions of the Lamé system, as explained in section 3 . Using singular functions, we are able to derive precise asymptotic formulas for $\nabla \mathbf{u}$ as $\epsilon \rightarrow 0$. As a consequence we are able to reprove that $\epsilon^{-1 / 2}$ is indeed an upper bound on the blow-up rate. Moreover, the asymptotic formulas enable to show that $\epsilon^{-1 / 2}$ is a lower bound on the blow-up rate as well in some cases, as presented in section 6. We emphasize that the asymptotic formulas are obtained using the variational principle, which is possible only because $\mathbf{q}_{j}$ are solutions of the Lamé system.

\subsection{Preliminary estimates of boundary integrals}

In this subsection, we characterize asymptotic behaviors of the following boundary integrals as $\epsilon \rightarrow 0$ :

$$
\int_{\partial D_{i}} \partial_{\nu} \mathbf{q}_{j} \cdot \Psi_{k}, \quad \int_{\partial D^{e}} \partial_{\nu} \mathbf{q}_{j} \cdot \mathbf{q}_{k}
$$

These integrals appear in later sections.

We first prove the following lemma.

Lemma 4.8. (i) For $k=1,2$, we have

$$
\int_{\partial D_{i}} \partial_{\nu} \mathbf{q}_{j} \cdot \Psi_{k}=(-1)^{i+1} \delta_{j k}, \quad i, j=1,2
$$

(ii) For $k=3$, we have

$$
\begin{aligned}
& \int_{\partial D_{i}} \partial_{\nu} \mathbf{q}_{1} \cdot \Psi_{3}=0, \\
& \int_{\partial D_{i}} \partial_{\nu} \mathbf{q}_{2} \cdot \Psi_{3}=(-1)^{i+1} a\left(-1+4 \pi \alpha_{2} \mu\right),
\end{aligned}
$$

for $i=1,2$, where $a$ is the constant defined by (2.47).

Proof. Suppose that $k=1,2$. Since $\mathcal{L}_{\lambda, \mu} \boldsymbol{\Gamma}\left(\mathbf{x}-\mathbf{p}_{l}\right) \mathbf{e}_{j}=\delta\left(\mathbf{x}-\mathbf{p}_{l}\right) \mathbf{e}_{j}$, Green's formula yields

$$
\int_{\partial D_{i}} \partial_{\nu_{\mathbf{x}}} \boldsymbol{\Gamma}\left(\mathbf{x}-\mathbf{p}_{l}\right) \mathbf{e}_{j} \cdot \Psi_{k} d \sigma(\mathbf{x})=\int_{D_{i}} \mathcal{L}_{\lambda, \mu} \boldsymbol{\Gamma}\left(\mathbf{x}-\mathbf{p}_{l}\right) \mathbf{e}_{j} \cdot \Psi_{k} d \sigma(\mathbf{x})=\delta_{i l} \delta_{j k} .
$$

Green's formula also yields

$$
\int_{\partial D_{i}} \partial_{\nu_{\mathbf{x}}}\left(\frac{\mathbf{x}-\mathbf{p}_{l}}{\left|\mathbf{x}-\mathbf{p}_{l}\right|^{2}}\right) \cdot \Psi_{k} d \sigma(\mathbf{x})=0
$$

In fact, if $i=l$, then we apply Green's formula to $\mathbb{R}^{2} \backslash D_{i}$, and to $D_{i}$ if $i \neq l$. So, 4.34 follows from 3.8 and 3.9 .

We now prove 4.35 and $(4.36)$ when $i=1$. The case when $i=2$ can be proved in the same way. Let us prove (4.36) first. In view of the definition (3.9) of $\mathbf{q}_{2}$, we have

$$
\int_{\partial D_{1}} \partial_{\nu} \mathbf{q}_{2} \cdot \Psi_{3}=\int_{\partial D_{1}} \partial_{\nu_{\mathbf{x}}}\left(\boldsymbol{\Gamma}\left(\mathbf{x}-\mathbf{p}_{1}\right) \mathbf{e}_{2}\right) \cdot \Psi_{3}-\alpha_{2} a \int_{\partial D_{1}} \partial_{\nu_{\mathbf{x}}}\left(\frac{\left(\mathbf{x}-\mathbf{p}_{1}\right)^{\perp}}{\left|\mathbf{x}-\mathbf{p}_{1}\right|^{2}}\right) \cdot \Psi_{3} .
$$


Since $\mathcal{L}_{\lambda, \mu}\left(\boldsymbol{\Gamma}\left(\mathbf{x}-\mathbf{p}_{1}\right) \mathbf{e}_{2}\right)=\delta_{\mathbf{p}_{1}}(\mathbf{x}) \mathbf{e}_{2}$, one can see that

$$
\int_{\partial D_{1}} \partial_{\nu_{\mathbf{x}}}\left(\boldsymbol{\Gamma}\left(\mathbf{x}-\mathbf{p}_{1}\right) \mathbf{e}_{2}\right) \cdot \Psi_{3}=\mathbf{e}_{2} \cdot \Psi_{3}\left(\mathbf{p}_{1}\right)=-a
$$

where the last equality holds because $\mathbf{p}_{1}=(-a, 0)$.

By using a change of variables $\mathbf{x} \rightarrow \mathbf{x}+\mathbf{p}_{1}$ and the fact that $\Psi_{3}\left(\mathbf{x}+\mathbf{p}_{1}\right)=\Psi_{3}(\mathbf{x})-a \Psi_{2}$, we obtain

$$
\begin{aligned}
& \int_{\partial D_{1}} \partial_{\nu_{\mathbf{x}}}\left(\frac{\left(\mathbf{x}-\mathbf{p}_{1}\right)^{\perp}}{\left|\mathbf{x}-\mathbf{p}_{1}\right|^{2}}\right) \cdot \Psi_{3}(\mathbf{x})=\int_{\partial D_{1}-\mathbf{p}_{1}} \partial_{\nu_{\mathbf{x}}}\left(\frac{\mathbf{x}^{\perp}}{|\mathbf{x}|^{2}}\right) \cdot \Psi_{3}\left(\mathbf{x}+\mathbf{p}_{1}\right) \\
& =-a \int_{\partial D_{1}-\mathbf{p}_{1}} \partial_{\nu_{\mathbf{x}}}\left(\frac{\mathbf{x}^{\perp}}{|\mathbf{x}|^{2}}\right) \cdot \Psi_{2}+\int_{\partial D_{1}-\mathbf{p}_{1}} \partial_{\nu_{\mathbf{x}}}\left(\frac{\mathbf{x}^{\perp}}{|\mathbf{x}|^{2}}\right) \cdot \Psi_{3}(\mathbf{x})
\end{aligned}
$$

One can show as before that

$$
\int_{\partial D_{1}-\mathbf{p}_{1}} \partial_{\nu_{\mathbf{x}}}\left(\frac{\mathbf{x}^{\perp}}{|\mathbf{x}|^{2}}\right) \cdot \Psi_{2} d \sigma(\mathbf{x})=0 .
$$

Let $B$ be a disk centered at 0 such that $\partial D_{1}-\mathbf{p}_{1} \subset B$. Then Green's formula yields

$$
\int_{\partial D_{1}-\mathbf{p}_{1}} \partial_{\nu_{\mathbf{x}}}\left(\frac{\mathbf{x}^{\perp}}{|\mathbf{x}|^{2}}\right) \cdot \Psi_{3}(\mathbf{x})=\int_{\partial B} \partial_{\nu_{\mathbf{x}}}\left(\frac{\mathbf{x}^{\perp}}{|\mathbf{x}|^{2}}\right) \cdot \Psi_{3}(\mathbf{x}) .
$$

Straightforward computations show that

$$
\partial_{\nu_{\mathbf{x}}}\left(\frac{\mathbf{x}^{\perp}}{|\mathbf{x}|^{2}}\right)=(-2 \mu) \frac{\mathbf{x}^{\perp}}{|\mathbf{x}|^{3}} \quad \text { for } \mathbf{x} \in \partial B
$$

So, we have

$$
\int_{\partial D_{1}-\mathbf{p}_{1}} \partial_{\nu_{\mathbf{x}}}\left(\frac{\mathbf{x}^{\perp}}{|\mathbf{x}|^{2}}\right) \cdot \Psi_{3}(\mathbf{x})=\int_{\partial B} \partial_{\nu_{\mathbf{x}}}\left(\frac{\mathbf{x}^{\perp}}{|\mathbf{x}|^{2}}\right) \cdot \mathbf{x}^{\perp}=\int_{\partial B}(-2 \mu) \frac{1}{|\mathbf{x}|}=-4 \pi \mu .
$$

It then follows from 4.39 and 4.40 that

$$
\int_{\partial D_{1}} \partial_{\nu_{\mathbf{x}}}\left(\frac{\left(\mathbf{x}-\mathbf{p}_{1}\right)^{\perp}}{\left|\mathbf{x}-\mathbf{p}_{1}\right|^{2}}\right) \cdot \Psi_{3}(\mathbf{x})=-4 \pi \mu .
$$

Combining 4.37)-4.41, we obtain 4.36).

We now prove 4.35 ). Like 4.38 we have

$$
\int_{\partial D_{1}} \partial_{\nu_{\mathbf{x}}}\left(\boldsymbol{\Gamma}\left(\mathbf{x}-\mathbf{p}_{1}\right) \mathbf{e}_{1}\right) \cdot \Psi_{3}=\mathbf{e}_{1} \cdot \Psi_{3}\left(\mathbf{p}_{1}\right)=0 .
$$

In the same way to show (4.41) one can show that

$$
\int_{\partial D_{1}} \partial_{\nu_{\mathbf{x}}}\left(\frac{\mathbf{x}-\mathbf{p}_{1}}{\left|\mathbf{x}-\mathbf{p}_{1}\right|^{2}}\right) \cdot \Psi_{3}=0
$$

Therefore, from the definition (3.8) of $\mathbf{q}_{1}$, we have 4.35), and the proof is completed. 
Lemma 4.9. We have

$$
\begin{aligned}
& \int_{\partial D^{e}} \partial_{\nu} \mathbf{q}_{j} \cdot \mathbf{q}_{j}=-m_{j}^{-1} \sqrt{\epsilon}+O(\tau \epsilon|\ln \epsilon|+\epsilon), \quad j=1,2, \\
& \int_{\partial D^{e}} \partial_{\nu} \mathbf{q}_{1} \cdot \mathbf{q}_{2}=O(\tau \epsilon|\ln \epsilon|+\epsilon) .
\end{aligned}
$$

Before proving Lemma 4.9, we need to estimate the conormal derivatives $\partial_{\nu} \mathbf{q}_{j}$ on $\partial D_{1} \cup \partial D_{2}$. We have the following lemma.

Lemma 4.10. For $\mathbf{x}=(x, y) \in\left(\partial D_{1} \cup \partial D_{2}\right) \cap \partial \Pi_{L_{0}}$, we have

$$
\left|\partial_{\nu} \mathbf{q}_{1}(\mathbf{x}) \cdot \mathbf{e}_{1}\right| \lesssim \frac{\sqrt{\epsilon}}{\epsilon+y^{2}}, \quad\left|\partial_{\nu} \mathbf{q}_{1}(\mathbf{x}) \cdot \mathbf{e}_{2}\right| \lesssim \frac{\sqrt{\epsilon}|y|}{\epsilon+y^{2}}+\sqrt{\epsilon}
$$

and

$$
\left|\partial_{\nu} \mathbf{q}_{2}(\mathbf{x}) \cdot \mathbf{e}_{1}\right| \lesssim \frac{\sqrt{\epsilon}|y|}{\epsilon+y^{2}}+\sqrt{\epsilon}, \quad\left|\partial_{\nu} \mathbf{q}_{2}(\mathbf{x}) \cdot \mathbf{e}_{2}\right| \lesssim \frac{\sqrt{\epsilon}}{\epsilon+y^{2}}
$$

Proof. We prove 4.44 only. 4.45 can be proved similarly. Let $\boldsymbol{\sigma}^{1}=\left(\sigma_{i j}^{1}\right)_{i, j=1}^{2}$ be the stress tensor of $\mathbf{q}_{1}$, namely, $\boldsymbol{\sigma}^{1}:=\mathbb{C} \widehat{\nabla} \mathbf{q}_{1}$. According to 2.4), the entries of $\boldsymbol{\sigma}^{1}$ can be written as

$$
\begin{aligned}
& \sigma_{11}^{1}=(\lambda+2 \mu) \partial_{1} q_{11}+\lambda \partial_{2} q_{12}, \\
& \sigma_{22}^{1}=\lambda \partial_{1} q_{11}+(\lambda+2 \mu) \partial_{2} q_{12}, \\
& \sigma_{12}^{1}=\sigma_{21}^{k}=\mu\left(\partial_{2} q_{11}+\partial_{1} q_{12}\right) .
\end{aligned}
$$

Thus we have the following estimates from Lemma 3.4 .

$$
\left|\sigma_{11}^{1}\right|+\left|\sigma_{22}^{1}\right| \lesssim \frac{\sqrt{\epsilon}}{\epsilon+y^{2}}, \quad\left|\sigma_{12}^{1}\right| \lesssim \frac{\sqrt{\epsilon}|y|}{\epsilon+y^{2}}+\sqrt{\epsilon} \quad \text { for }(x, y) \in \Pi_{L_{0}} .
$$

Note that $\partial_{\nu} \mathbf{q}_{1}=\boldsymbol{\sigma}^{1} \mathbf{n}$ and the outward unit normal vector $\mathbf{n}$ on $\partial D_{i} \cap \partial \Pi_{L_{0}}$ is given as follows:

$$
\mathbf{n}=\frac{1}{\sqrt{1+\left(f_{i}^{\prime}(y)\right)^{2}}}\left((-1)^{i+1}, f_{i}^{\prime}(y)\right), \quad i=1,2 .
$$

Moreover, we have $\left|f_{i}^{\prime}(y)\right| \lesssim|y|$. Therefore, we obtain

$$
\begin{aligned}
\left|\partial_{\nu} \mathbf{q}_{1}(x, y) \cdot \mathbf{e}_{1}\right| & =\left|\left(\boldsymbol{\sigma}^{1} \mathbf{n}\right)_{1}\right|=\left|\frac{1}{\sqrt{1+\left(f_{i}^{\prime}(y)\right)^{2}}}\left((-1)^{i+1} \sigma_{11}^{1}+f_{i}^{\prime}(y) \sigma_{12}^{1}\right)\right| \\
& \lesssim \frac{\sqrt{\epsilon}}{\epsilon+y^{2}}+\frac{\sqrt{\epsilon} y^{2}}{\epsilon+y^{2}} \lesssim \frac{\sqrt{\epsilon}}{\epsilon+y^{2}}
\end{aligned}
$$

and

$$
\begin{aligned}
\left|\partial_{\nu} \mathbf{q}_{1}(x, y) \cdot \mathbf{e}_{2}\right| & =\left|\left(\boldsymbol{\sigma}^{1} \mathbf{n}\right)_{2}\right|=\left|\frac{1}{\sqrt{1+\left(f_{i}^{\prime}(y)\right)^{2}}}\left((-1)^{i+1} \sigma_{12}^{1}+f_{i}^{\prime}(y) \sigma_{22}^{1}\right)\right| \\
& \lesssim \frac{\sqrt{\epsilon}|y|}{\epsilon+y^{2}}+\sqrt{\epsilon}
\end{aligned}
$$


for $\mathbf{x}=(x, y) \in \partial D_{i} \cap \partial \Pi_{L_{0}}$ and $i=1,2$. The proof is completed.

Proof of Lemma 4.9. To prove 4.42, we write

$$
\begin{aligned}
\int_{\partial D_{1} \cup \partial D_{2}} \partial_{\nu} \mathbf{q}_{1} \cdot \mathbf{q}_{1}= & \sum_{i=1}^{2}(-1)^{i}\left(\alpha_{1}-\alpha_{2}\right) \kappa_{i} a \int_{\partial D_{i}} \partial_{\nu} \mathbf{q}_{1} \cdot \Psi_{1} \\
& +\sum_{i=1}^{2} \int_{\partial D_{i}} \partial_{\nu} \mathbf{q}_{1} \cdot\left[\mathbf{q}_{1}-(-1)^{i}\left(\alpha_{1}-\alpha_{2}\right) \kappa_{i} a \Psi_{1}\right] .
\end{aligned}
$$

By Lemma 4.8 (i), we have

$$
\sum_{i=1}^{2}(-1)^{i}\left(\alpha_{1}-\alpha_{2}\right) \kappa_{i} a \int_{\partial D_{i}} \partial_{\nu} \mathbf{q}_{1} \cdot \Psi_{1}=-\left(\alpha_{1}-\alpha_{2}\right)\left(\kappa_{1}+\kappa_{2}\right) a .
$$

Then 2.48 and 3.12 yield

$$
\sum_{i=1}^{2}(-1)^{i}\left(\alpha_{1}-\alpha_{2}\right) \kappa_{i} a \int_{\partial D_{i}} \partial_{\nu} \mathbf{q}_{1} \cdot \Psi_{1}=-m_{1}^{-1} \sqrt{\epsilon}+O\left(\epsilon^{3 / 2}\right) .
$$

It then remains to show that

$$
\int_{\partial D_{i}} \partial_{\nu} \mathbf{q}_{1} \cdot\left[\mathbf{q}_{1}-(-1)^{i}\left(\alpha_{1}-\alpha_{2}\right) \kappa_{i} a \Psi_{1}\right]=O(\tau \epsilon|\ln \epsilon|+\epsilon), \quad i=1,2 .
$$

To prove 4.46), let us write

$$
\int_{\partial D_{1}} \partial_{\nu} \mathbf{q}_{1} \cdot\left[\mathbf{q}_{1}-(-1)\left(\alpha_{1}-\alpha_{2}\right) \kappa_{1} a \Psi_{1}\right]=\int_{\partial D_{1} \cap \partial \Pi_{L_{0}}}+\int_{\partial D_{1} \backslash \partial \Pi_{L_{0}}}:=I_{1}+I_{2} .
$$

From Lemma 3.6 and the fact that $a \approx \sqrt{\epsilon}$, we see that $\left|I_{2}\right| \lesssim \epsilon$. Note that

$$
\left|I_{1}\right| \leq \int_{\partial D_{1} \cap \partial \Pi_{L_{0}}}\left|\partial_{\nu} \mathbf{q}_{1} \cdot \mathbf{e}_{1}\left(q_{11}+\left(\alpha_{1}-\alpha_{2}\right) \kappa_{1} a\right)\right|+\left|\partial_{\nu} \mathbf{q}_{1} \cdot \mathbf{e}_{2} q_{12}\right| .
$$

From 3.60 and (3.61), we see that

$$
\left|q_{11}+\left(\alpha_{1}-\alpha_{2}\right) \kappa_{1} a\right| \lesssim \epsilon^{3 / 2}+\sqrt{\epsilon} y^{2}+\tau \sqrt{\epsilon}|y|
$$

and

$$
\left|q_{12}\right| \lesssim \sqrt{\epsilon}|y|
$$

It then follows from Lemma 4.10 that

$$
\begin{aligned}
\left|I_{1}\right| & \lesssim \int_{\partial D_{1} \cap \partial \Pi_{L_{0}}} \frac{\sqrt{\epsilon}}{\epsilon+y^{2}}\left(\epsilon^{3 / 2}+\sqrt{\epsilon} y^{2}+\tau \sqrt{\epsilon}|y|\right)+\left(\frac{\sqrt{\epsilon}|y|}{\epsilon+y^{2}}+\sqrt{\epsilon}\right) \sqrt{\epsilon}|y| \\
& \lesssim \int_{-L_{0}}^{L_{0}} \frac{\epsilon \tau|y|}{\epsilon+y^{2}} d y+\epsilon \lesssim \tau \epsilon|\ln \epsilon|+\epsilon
\end{aligned}
$$


This proves 4.46) for $i=1$. The case for $i=2$ can be proved in the same way. So, 4.42 is proved.

Next we prove 4.43). Thanks to (4.34) with $j=1$ and $k=2$, we can write

$$
\begin{aligned}
\int_{\partial D^{e}} \partial_{\nu} \mathbf{q}_{1} \cdot \mathbf{q}_{2}= & \sum_{i=1}^{2} \alpha_{2} \kappa_{i}^{2} a \int_{\partial D_{i}} \partial_{\nu} \mathbf{q}_{1} \cdot y \Psi_{1} \\
& \left.+\sum_{i=1}^{2} \int_{\partial D_{i}} \partial_{\nu} \mathbf{q}_{1} \cdot\left[\mathbf{q}_{2}-\alpha_{2} \kappa_{i}^{2} a y \Psi_{1}-(-1)^{i}\left(\alpha_{1}+\alpha_{2}\right) \kappa_{i} a \Psi_{2}\right)\right]
\end{aligned}
$$

Green's formula yields

$$
\begin{aligned}
\int_{\partial D_{i}} \partial_{\nu} \mathbf{q}_{1} \cdot y \Psi_{1} & =\int_{\partial D_{i}} \partial_{\nu} \mathbf{q}_{1} \cdot y \Psi_{1}-\int_{\partial D_{i}} \partial_{\nu}\left(y \Psi_{1}\right) \cdot \mathbf{q}_{1}+\int_{\partial D_{i}} \partial_{\nu}\left(y \Psi_{1}\right) \cdot \mathbf{q}_{1} \\
& =\int_{D_{i}} \mathcal{L}_{\lambda, \mu} \mathbf{q}_{1} \cdot y \Psi_{1}-\int_{D_{i}} \mathcal{L}_{\lambda, \mu}\left(y \Psi_{1}\right) \cdot \mathbf{q}_{1}+\int_{\partial D_{i}} \partial_{\nu}\left(y \Psi_{1}\right) \cdot \mathbf{q}_{1} \\
& =\int_{D_{i}} \mathcal{L}_{\lambda, \mu} \mathbf{q}_{1} \cdot y \Psi_{1}+\int_{\partial D_{i}} \partial_{\nu}\left(y \Psi_{1}\right) \cdot \mathbf{q}_{1} .
\end{aligned}
$$

Observe from (3.5) and the definition (3.8) of $\mathbf{q}_{1}$ that

$$
\mathcal{L}_{\lambda, \mu} \mathbf{q}_{1}=\left(\delta_{\mathbf{p}_{1}}-\delta_{\mathbf{p}_{2}}\right) \mathbf{e}_{1}+\sum_{j=1}^{2} \frac{\alpha_{2} a}{\alpha_{1}-\alpha_{2}}\left(\partial_{1} \delta_{\mathbf{p}_{j}} \mathbf{e}_{1}+\partial_{2} \delta_{\mathbf{p}_{j}} \mathbf{e}_{2}\right)
$$

where $\delta_{\mathbf{p}_{j}}$ denotes the Dirac delta at $\mathbf{p}_{j}$. So, we see that

$$
\int_{D_{i}} \mathcal{L}_{\lambda, \mu} \mathbf{q}_{1} \cdot y \Psi_{1}=0
$$

It follows from Lemma 3.6 and Lemma 3.8 that $\left\|\mathbf{q}_{1}\right\|_{L^{\infty}\left(\partial D_{i}\right)} \lesssim \sqrt{\epsilon}$ for $i=1,2$. So we have

$$
\int_{\partial D_{i}} \partial_{\nu}\left(y \Psi_{1}\right) \cdot \mathbf{q}_{1}=O(\sqrt{\epsilon})
$$

and hence

$$
\int_{\partial D_{i}} \partial_{\nu} \mathbf{q}_{1} \cdot y \Psi_{1}=O(\sqrt{\epsilon}), \quad i=1,2
$$

Let

$$
\begin{aligned}
\left.\int_{\partial D_{1}} \partial_{\nu} \mathbf{q}_{1} \cdot\left[\mathbf{q}_{2}-\alpha_{2} \kappa_{1}^{2} a y \Psi_{1}+\left(\alpha_{1}+\alpha_{2}\right) \kappa_{1} a \Psi_{2}\right)\right] & =\int_{\partial D_{1} \cap \partial \Pi_{L_{0}}}+\int_{\partial D_{1} \backslash \partial \Pi_{L_{0}}} \\
& :=J_{1}+J_{2} .
\end{aligned}
$$

As before, from Lemma 3.6 and the fact that $a \approx \sqrt{\epsilon}$, we see that $\left|J_{2}\right| \lesssim \epsilon$. From Lemma 
3.8. Lemma 4.10 and the fact that $a \approx \sqrt{\epsilon}$, we have

$$
\begin{aligned}
\left|J_{1}\right| & \lesssim \int_{\partial D_{1} \cap \partial \Pi_{L_{0}}}\left|\partial_{\nu} \mathbf{q}_{1} \cdot \Psi_{1}\left(q_{21}-\alpha_{2} \kappa_{1}^{2} a y\right)\right|+\left|\partial_{\nu} \mathbf{q}_{1} \cdot \Psi_{2}\left(q_{22}+\left(\alpha_{1}+\alpha_{2}\right) \kappa_{1} a\right)\right| \\
& \lesssim \int_{\partial D_{1} \cap \partial \Pi_{L_{0}}}\left(\frac{\sqrt{\epsilon}}{\epsilon+y^{2}}+\frac{\sqrt{\epsilon}|y|}{\epsilon+y^{2}}+\sqrt{\epsilon}\right)\left(\epsilon^{3 / 2}+\sqrt{\epsilon} y^{2}+\tau \sqrt{\epsilon}|y|\right) \\
& \lesssim \int_{-L_{0}}^{L_{0}} \frac{\tau \epsilon|y|}{\epsilon+y^{2}} d y+\epsilon \\
& \lesssim \tau \epsilon|\ln \epsilon|+\epsilon
\end{aligned}
$$

So we obtain

$$
\left|\int_{\partial D_{1}} \partial_{\nu} \mathbf{q}_{1} \cdot\left(\mathbf{q}_{2}-\alpha_{2} \kappa_{1}^{2} a y \Psi_{1}+\left(\alpha_{1}+\alpha_{2}\right) \kappa_{1} a \Psi_{2}\right)\right| \lesssim \tau \epsilon|\ln \epsilon|+\epsilon .
$$

Similarly, one can see that

$$
\left|\int_{\partial D_{2}} \partial_{\nu} \mathbf{q}_{1} \cdot\left(\mathbf{q}_{2}-\alpha_{2} \kappa_{2}^{2} a y \Psi_{1}-\left(\alpha_{1}+\alpha_{2}\right) \kappa_{2} a \Psi_{2}\right)\right| \lesssim \tau \epsilon|\ln \epsilon|+\epsilon .
$$

Since $a \approx \sqrt{\epsilon}, 4.43$ follows from 4.47) and 4.49-4.51. The proof is completed.

\subsection{Proof of Lemma 4.1}

We first show that

$$
\mathcal{I}_{j k}=\int_{\partial D_{1}} \partial_{\nu} \mathbf{h}_{j} \cdot \Psi_{k}=-\int_{\partial D_{2}} \partial_{\nu} \mathbf{h}_{j} \cdot \Psi_{k}, \quad j=1,2, k=1,2,3 .
$$

In fact, we see from Lemma 2.8 and the boundary conditions of $\mathbf{h}_{j}$ that

$$
\mathcal{I}_{j k}=-\int_{\partial D^{e}} \partial_{\nu} \mathbf{h}_{j} \cdot \mathbf{h}_{k}=\frac{1}{2} \int_{\partial D_{1}} \partial_{\nu} \mathbf{h}_{j} \cdot \Psi_{k}-\frac{1}{2} \int_{\partial D_{2}} \partial_{\nu} \mathbf{h}_{j} \cdot \Psi_{k} .
$$

Since $\widehat{\nabla} \Psi_{k}=0$, we obtain using Lemma 2.8 that

$$
\int_{\partial D^{e}} \partial_{\nu} \mathbf{h}_{j} \cdot \Psi_{k}=-\int_{D^{e}} \mathbb{C} \widehat{\nabla} \mathbf{h}_{j}: \widehat{\nabla} \Psi_{k}=0
$$

So, 4.52 follows.

Since $\mathcal{L}_{\lambda, \mu} \mathbf{H}_{\Omega}=0$ in $D_{i}$, we have

$$
\int_{\partial D_{i}} \partial_{\nu} \mathbf{H}_{\Omega} \cdot \mathbf{h}_{j}=(-1)^{i} \frac{1}{2} \int_{\partial D_{i}} \partial_{\nu} \mathbf{H}_{\Omega} \cdot \Psi_{j}=0
$$

Thus we have

$$
\mathcal{J}_{\Omega, j}=\left.\int_{\partial D^{e}} \partial_{\nu} \mathbf{h}_{j}\right|_{+} \cdot \mathbf{H}_{\Omega}=\left.\int_{\partial D^{e}} \partial_{\nu} \mathbf{h}_{j}\right|_{+} \cdot \mathbf{H}_{\Omega}-\partial_{\nu} \mathbf{H}_{\Omega} \cdot \mathbf{h}_{j}
$$


One can easily see from 2.18 and Lemma 2.6 (i) that $\mathbf{u}-\mathbf{H}_{\Omega}$ can be extended to $D^{e}$ so that the extended function, still denoted by $\mathbf{u}-\mathbf{H}_{\Omega}$, satisfies $\mathcal{L}_{\lambda, \mu}\left(\mathbf{u}-\mathbf{H}_{\Omega}\right)=0$ in $D^{e}$ and $\mathbf{u}-\mathbf{H}_{\Omega} \in \mathcal{A}$. Therefore, we have from Lemma 2.8

$$
\left.\int_{\partial D^{e}}\left(\mathbf{u}-\mathbf{H}_{\Omega}\right) \cdot \partial_{\nu} \mathbf{h}_{j}\right|_{+}-\left.\partial_{\nu}\left(\mathbf{u}-\mathbf{H}_{\Omega}\right)\right|_{+} \cdot \mathbf{h}_{j}=0 .
$$

We then infer from (2.7) and (4.54) that

$$
\mathcal{J}_{\Omega, j}=\left.\int_{\partial D^{e}} \partial_{\nu} \mathbf{h}_{j}\right|_{+} \cdot \mathbf{u}-\left.\partial_{\nu} \mathbf{u}\right|_{+} \cdot \mathbf{h}_{j}=\left.\int_{\partial D^{e}} \partial_{\nu} \mathbf{h}_{j}\right|_{+} \cdot \mathbf{u} .
$$

Then the boundary condition in 2.6 and 4.52 yield

$$
\begin{aligned}
\mathcal{J}_{\Omega, j} & =\left.\sum_{k=1}^{3} c_{1 k} \int_{\partial D_{1}} \partial_{\nu} \mathbf{h}_{j}\right|_{+} \cdot \Psi_{k}+\left.c_{2 k} \int_{\partial D_{2}} \partial_{\nu} \mathbf{h}_{j}\right|_{+} \cdot \Psi_{k} \\
& =\sum_{k=1}^{3}\left(c_{1 k}-c_{2 k}\right) \mathcal{I}_{j k} .
\end{aligned}
$$

So, 4.6 follows.

\subsection{Estimates of integrals $\mathcal{I}_{j k}$ and $\mathcal{J}_{k}$ and proof of Proposition 4.2}

In this subsection we derive estimates of the integrals $\mathcal{I}_{j k}$ and $\mathcal{J}_{\Omega, k}$, and prove Proposition 4.2 as a consequence. Some of estimates obtained in this subsection will be used in the next subsection as well.

Lemma 4.11. The following holds:

$$
\begin{aligned}
& \mathcal{I}_{11}=m_{1} \epsilon^{-1 / 2}+O(\tau|\ln \epsilon|+1), \\
& \mathcal{I}_{12}=O(\tau|\ln \epsilon|+1), \\
& \mathcal{I}_{22}=m_{2} \epsilon^{-1 / 2}+O(\tau|\ln \epsilon|+1),
\end{aligned}
$$

as $\epsilon \rightarrow 0$.

Proof. According to 3.82, we have

$$
\begin{aligned}
\mathcal{I}_{j k} & =\int_{D^{e}} \mathbb{C} \widehat{\nabla} \mathbf{h}_{j}: \widehat{\nabla} \mathbf{h}_{k}=\int_{D^{e}} \mathbb{C} \widehat{\nabla}\left(\frac{m_{j}}{\sqrt{\epsilon}} \mathbf{q}_{j}+\mathbf{r}_{j}\right): \widehat{\nabla} \mathbf{h}_{k} \\
& =\frac{m_{j}}{\sqrt{\epsilon}} \int_{D^{e}} \mathbb{C} \widehat{\nabla} \mathbf{q}_{j}: \widehat{\nabla} \mathbf{h}_{k}+\int_{D^{e}} \mathbb{C} \widehat{\nabla} \mathbf{r}_{j}: \widehat{\nabla} \mathbf{h}_{k} \\
& =\frac{m_{j}}{\sqrt{\epsilon}} \int_{D^{e}} \mathbb{C} \widehat{\nabla} \mathbf{q}_{j}: \widehat{\nabla} \mathbf{h}_{k}+\int_{D^{e}} \mathbb{C} \widehat{\nabla} \mathbf{r}_{j}: \widehat{\nabla}\left(\frac{m_{k}}{\sqrt{\epsilon}} \mathbf{q}_{k}+\mathbf{r}_{k}\right) \\
& =\frac{m_{j}}{\sqrt{\epsilon}} \int_{D^{e}} \mathbb{C} \widehat{\nabla} \mathbf{q}_{j}: \widehat{\nabla} \mathbf{h}_{k}+\frac{m_{k}}{\sqrt{\epsilon}} \int_{D^{e}} \mathbb{C} \widehat{\nabla} \mathbf{r}_{j}: \widehat{\nabla} \mathbf{q}_{k}+\int_{D^{e}} \mathbb{C} \widehat{\nabla} \mathbf{r}_{j}: \widehat{\nabla} \mathbf{r}_{k} .
\end{aligned}
$$


Since

$$
\begin{aligned}
\frac{m_{k}}{\sqrt{\epsilon}} \int_{D^{e}} \mathbb{C} \hat{\nabla} \mathbf{r}_{j}: \hat{\nabla} \mathbf{q}_{k} & =\frac{m_{k}}{\sqrt{\epsilon}} \int_{D^{e}} \mathbb{C} \widehat{\nabla}\left(\mathbf{h}_{j}-\frac{m_{j}}{\sqrt{\epsilon}} \mathbf{q}_{j}\right): \hat{\nabla} \mathbf{q}_{k} \\
& =\frac{m_{k}}{\sqrt{\epsilon}} \int_{D^{e}} \mathbb{C} \widehat{\nabla} \mathbf{q}_{k}: \hat{\nabla} \mathbf{h}_{j}-\frac{m_{j} m_{k}}{\epsilon} \int_{D^{e}} \mathbb{C} \widehat{\nabla} \mathbf{q}_{j}: \widehat{\nabla} \mathbf{q}_{k}
\end{aligned}
$$

it follows that

$$
\begin{aligned}
\mathcal{I}_{j k}= & \frac{m_{j}}{\sqrt{\epsilon}} \int_{D^{e}} \mathbb{C} \widehat{\nabla} \mathbf{q}_{j}: \widehat{\nabla} \mathbf{h}_{k}+\frac{m_{k}}{\sqrt{\epsilon}} \int_{D^{e}} \mathbb{C} \widehat{\nabla} \mathbf{q}_{k}: \widehat{\nabla} \mathbf{h}_{j} \\
& -\frac{m_{j} m_{k}}{\epsilon} \int_{D^{e}} \mathbb{C} \widehat{\nabla} \mathbf{q}_{j}: \widehat{\nabla} \mathbf{q}_{k}+\int_{D^{e}} \mathbb{C} \widehat{\nabla} \mathbf{r}_{j}: \widehat{\nabla} \mathbf{r}_{k}
\end{aligned}
$$

Then Lemma 2.8 yields

$$
\begin{aligned}
\mathcal{I}_{j k}=- & \frac{m_{j}}{\sqrt{\epsilon}} \int_{\partial D^{e}} \partial_{\nu} \mathbf{q}_{j} \cdot \mathbf{h}_{k}-\frac{m_{k}}{\sqrt{\epsilon}} \int_{\partial D^{e}} \partial_{\nu} \mathbf{q}_{k} \cdot \mathbf{h}_{j} \\
& +\frac{m_{j} m_{k}}{\epsilon} \int_{\partial D^{e}} \partial_{\nu} \mathbf{q}_{j} \cdot \mathbf{q}_{k}+\int_{D^{e}} \mathbb{C} \widehat{\nabla} \mathbf{r}_{j}: \widehat{\nabla} \mathbf{r}_{k} .
\end{aligned}
$$

Now, 4.55-4.57 follow from Lemma 4.8 and 4.9. In fact, we have from Proposition 3.13 that

$$
\left|\int_{D^{e}} \mathbb{C} \widehat{\nabla} \mathbf{r}_{j}: \widehat{\nabla} \mathbf{r}_{k}\right| \lesssim \mathcal{E}\left[\mathbf{r}_{j}\right]^{1 / 2} \mathcal{E}\left[\mathbf{r}_{k}\right]^{1 / 2} \lesssim 1
$$

Since $\mathbf{h}_{j}=(-1)^{i} \frac{1}{2} \Psi_{j}$ on $\partial D_{i}$, we have

$$
\begin{aligned}
\mathcal{I}_{j k}= & -\frac{m_{j}}{\sqrt{\epsilon}} \sum_{i=1}^{2} \frac{(-1)^{i}}{2} \int_{\partial D_{i}} \partial_{\nu} \mathbf{q}_{j} \cdot \Psi_{k} \\
& -\frac{m_{k}}{\sqrt{\epsilon}} \sum_{i=1}^{2} \frac{(-1)^{i}}{2} \int_{\partial D_{i}} \partial_{\nu} \mathbf{q}_{k} \cdot \Psi_{j}+\frac{m_{j} m_{k}}{\epsilon} \int_{\partial D^{e}} \partial_{\nu} \mathbf{q}_{j} \cdot \mathbf{q}_{k}+O(1) .
\end{aligned}
$$

Then, from 4.34, 4.42 and 4.43), we have

$$
\begin{aligned}
& \mathcal{I}_{11}=\frac{m_{1}^{2}}{\epsilon}\left(-\frac{\sqrt{\epsilon}}{m_{1}}+O(\tau \epsilon|\ln \epsilon|+\epsilon)\right)+2 \frac{m_{1}}{\sqrt{\epsilon}}+O(1)=\frac{m_{1}}{\sqrt{\epsilon}}+O(\tau|\ln \epsilon|+1), \\
& \mathcal{I}_{22}=\frac{m_{2}^{2}}{\epsilon}\left(-\frac{\sqrt{\epsilon}}{m_{2}}+O(\tau \epsilon|\ln \epsilon|+\epsilon)\right)+2 \frac{m_{2}}{\sqrt{\epsilon}}+O(1)=\frac{m_{2}}{\sqrt{\epsilon}}+O(\tau|\ln \epsilon|+1), \\
& \mathcal{I}_{12}=\frac{m_{1} m_{2}}{\epsilon} O(\tau \epsilon|\ln \epsilon|+\epsilon)+O(1)=O(\tau|\ln \epsilon|+1) .
\end{aligned}
$$

This completes the proof.

Lemma 4.12. We have

$$
\left|\mathcal{I}_{13}\right|,\left|\mathcal{I}_{23}\right| \lesssim 1
$$

and

$$
\mathcal{I}_{33} \approx 1
$$


Proof. We prove 4.59 first. For that we closely follow the proof of (4.12) in [9]. Let $\mathbf{h}_{3}^{K}$ be the function defined as follows: for $(x, y) \in \Pi_{L}$,

$$
\mathbf{h}_{3}^{K}(x, y)=\frac{x+f_{1}(y)}{f_{2}(y)+f_{1}(y)} \Psi_{3}+\frac{(-1)}{2} \Psi_{3} .
$$

We emphasize that

$$
\mathbf{h}_{3}^{K}=-\frac{1}{2} \mathbf{v}_{13}^{K}+\frac{1}{2} \mathbf{v}_{23}^{K},
$$

where $\mathbf{v}_{i 3}$ is defined by 4.31 . We then extend $\mathbf{h}_{3}^{K}$ to $D^{e} \backslash \Pi_{L}$ so that

$$
\left\{\begin{array}{l}
\mathbf{h}_{3}^{K}=(-1)^{i} \frac{1}{2} \Psi_{3} \quad \text { on } \partial D_{i}, i=1,2 \\
\left.\mathbf{h}_{3}^{K}\right|_{\mathbb{R}^{2} \backslash B_{0}}=0 \\
\left\|\mathbf{h}_{3}^{K}\right\|_{H^{1}\left(D^{e} \backslash \Pi_{L}\right)} \lesssim 1,
\end{array}\right.
$$

where $B_{0}$ is a disk which contains $\overline{D_{1} \cup D_{2}}$.

It is easy to see that, for $(x, y) \in \Pi_{L}$,

$$
\partial_{1} h_{31}^{K}=-\frac{y}{\epsilon+\frac{1}{2}\left(\kappa_{1}+\kappa_{2}\right) y^{2}}+O(1)
$$

and

$$
\partial_{2} h_{31}^{K}, \partial_{1} h_{32}^{K}, \partial_{2} h_{32}^{K}=O(1) .
$$

We mention that these estimates together with Lemma 3.11 show that $\nabla \mathbf{h}_{3}^{K}$ and $\nabla \mathbf{q}_{3}$ have the same behavior in $\Pi_{L}$. In fact, we have

$$
\left|\nabla \mathbf{q}_{3}-\nabla \mathbf{h}_{3}^{K}\right| \lesssim 1 \quad \text { in } \Pi_{L} .
$$

This estimate will be used in the proof of Proposition 4.5 .

By Lemma 2.9, we have

$$
\begin{aligned}
\mathcal{I}_{33} & =\mathcal{E}_{D^{e}}\left[\mathbf{h}_{3}\right] \leq \mathcal{E}_{D^{e}}\left[\mathbf{h}_{3}^{K}\right] \lesssim \int_{D^{e}}\left|\nabla \mathbf{h}_{3}^{K}\right|^{2} \\
& \lesssim \int_{\Pi_{L}}\left|\nabla \mathbf{h}_{3}^{K}\right|^{2}+\int_{D^{e} \backslash \Pi_{L}}\left|\nabla \mathbf{h}_{3}^{K}\right|^{2} \\
& \lesssim \int_{-L}^{L} \int_{-f_{1}(y)}^{f_{2}(y)}\left(\frac{|y|}{\epsilon+y^{2}}\right)^{2} d x d y+1 \\
& \lesssim \int_{-L}^{L} \frac{y^{2}}{\epsilon+y^{2}} d y+1 \lesssim 1,
\end{aligned}
$$

where the second to last inequality holds since $f_{2}(y)+f_{1}(y) \lesssim \epsilon+y^{2}$.

To prove the opposite inequality, we invoke a result in [9]: For any $\mathbf{v} \in H^{1}\left(\Pi_{L} \backslash \Pi_{L_{0}}\right)$ satisfying $\mathbf{v}=0$ on $\partial D_{1} \cap \partial\left(\Pi_{L} \backslash \Pi_{L_{0}}\right)$, it holds

$$
\int_{\Pi_{L} \backslash \overline{\Pi_{L_{0}}}}|\nabla \mathbf{v}|^{2} \lesssim \int_{\Pi_{L} \backslash \overline{\Pi_{L_{0}}}}|\widehat{\nabla} \mathbf{v}|^{2}
$$


(See the proof of (4.12) in 9].)

Let $\widetilde{\mathbf{h}}_{3}:=\mathbf{h}_{3}+\frac{1}{2} \Psi_{3}$. Then $\widetilde{\mathbf{h}}_{3}=0$ on $\partial D_{1} \cap \partial\left(\Pi_{L} \backslash \Pi_{L_{0}}\right)$ and $\widetilde{\mathbf{h}}_{3}=\Psi_{3}$ on $\partial D_{2} \cap \partial\left(\Pi_{L} \backslash\right.$ $\Pi_{L_{0}}$ ). Therefore, using 4.66, we have

$$
\mathcal{I}_{33}=\mathcal{E}_{D^{e}}\left[\mathbf{h}_{3}\right]=\mathcal{E}_{D^{e}}\left[\widetilde{\mathbf{h}}_{3}\right] \gtrsim \int_{\Pi_{L} \backslash \overline{\Pi_{L_{0}}}}\left|\nabla \widetilde{\mathbf{h}}_{3}\right|^{2} \gtrsim 1
$$

So, 4.59 is proved.

To prove (4.58), let $j=1$ or 2 . From Lemma 2.8 and 3.82 , we have

$$
\begin{aligned}
\mathcal{I}_{j 3} & =\frac{m_{j}}{\sqrt{\epsilon}} \int_{D^{e}} \widehat{\nabla} \mathbf{q}_{j}: \widehat{\nabla} \mathbf{h}_{3}+\int_{D^{e}} \mathbb{C} \widehat{\nabla} \mathbf{r}_{j}: \widehat{\nabla} \mathbf{h}_{3} \\
& =-\frac{m_{j}}{\sqrt{\epsilon}} \int_{\partial D^{e}} \partial_{\nu} \mathbf{q}_{j} \cdot \mathbf{h}_{3}+\int_{D^{e}} \mathbb{C} \widehat{\nabla} \mathbf{r}_{j}: \widehat{\nabla} \mathbf{h}_{3} \\
& =\frac{1}{2} \frac{m_{j}}{\sqrt{\epsilon}}\left(\int_{\partial D_{1}} \partial_{\nu} \mathbf{q}_{j} \cdot \Psi_{3}-\int_{\partial D_{2}} \partial_{\nu} \mathbf{q}_{j} \cdot \Psi_{3}\right)+\int_{D^{e}} \mathbb{C} \widehat{\nabla} \mathbf{r}_{j}: \widehat{\nabla} \mathbf{h}_{3} \\
& =I+I I .
\end{aligned}
$$

From Lemma 4.8 (ii) and the fact that $a \approx \sqrt{\epsilon}$, we have

$$
I=\frac{m_{j}}{\sqrt{\epsilon}} \delta_{2 j} a\left(-1+4 \pi \alpha_{2} \mu\right)=O(1) .
$$

It is clear from Proposition 3.13 and 4.59 that

$$
|I I| \lesssim \mathcal{E}_{D^{e}}\left[\mathbf{r}_{j}\right]^{1 / 2} \mathcal{I}_{33}{ }^{1 / 2} \lesssim 1
$$

This proves 4.58.

Lemma 4.13. We have

$$
\left|\mathcal{J}_{\Omega, k}\right| \lesssim\|\mathbf{g}\|_{C^{1, \gamma}(\partial \Omega)}, \quad k=1,2,3 .
$$

Before proving Lemma 4.13, let us make a short remark on regularity of $\mathbf{H}_{\Omega}$. Recall that $\mathbf{H}_{\Omega}$ is defined by

$$
\mathbf{H}_{\Omega}=-\mathcal{S}_{\partial \Omega}\left[\left.\partial_{\nu} \mathbf{u}\right|_{\partial \Omega}\right]+\mathcal{D}_{\partial \Omega}[\mathbf{g}] \text { in } \Omega .
$$

As shown in [9], we have

$$
\|\nabla \mathbf{u}\|_{L^{\infty}\left(\widetilde{\Omega} \backslash \Pi_{L}\right)} \lesssim\|\mathbf{g}\|_{C^{1, \gamma}(\partial \Omega)}
$$

In particular, we have

$$
\left\|\partial_{\nu} \mathbf{u}\right\|_{L^{\infty}(\partial \Omega)} \lesssim\|\mathbf{g}\|_{C^{1, \gamma}(\partial \Omega)} .
$$

So, for any $\Omega_{1}$ such that $\overline{\Omega_{1}} \subset \Omega$, we have

$$
\left\|\mathbf{H}_{\Omega}\right\|_{C^{2}\left(\Omega_{1}\right)} \lesssim\|\mathbf{g}\|_{C^{1, \gamma}(\partial \Omega)}
$$

We also have

$$
\left\|\mathbf{H}_{\Omega}\right\|_{H^{1}(\partial \Omega)} \lesssim\|\mathbf{g}\|_{C^{1, \gamma}(\partial \Omega)}
$$


Importance of these inequalities is that they hold independently of $\epsilon$.

Proof of Lemma 4.13. Let us first consider the case when $k=1,2$. For simplicity, we assume $\|\mathbf{g}\|_{C^{1, \gamma}(\partial \Omega)}=1$. Since $\int_{\partial D^{e}} \partial_{\nu} \mathbf{H}_{\Omega} \cdot \mathbf{h}_{k}=0$, we have

$$
\mathcal{J}_{\Omega, k}=\int_{\partial D^{e}} \partial_{\nu} \mathbf{h}_{k} \cdot \mathbf{H}_{\Omega}-\int_{\partial D^{e}} \partial_{\nu} \mathbf{H}_{\Omega} \cdot \mathbf{h}_{k} .
$$

Then (3.82) yields

$$
\begin{aligned}
\mathcal{J}_{\Omega, k} & =\frac{m_{k}}{\sqrt{\epsilon}}\left(\int_{\partial D^{e}} \partial_{\nu} \mathbf{q}_{k} \cdot \mathbf{H}_{\Omega}-\partial_{\nu} \mathbf{H}_{\Omega} \cdot \mathbf{q}_{k}\right)+\int_{\partial D^{e}} \partial_{\nu} \mathbf{r}_{k} \cdot \mathbf{H}_{\Omega}+(-1) \int_{\partial D^{e}} \partial_{\nu} \mathbf{H}_{\Omega} \cdot \mathbf{r}_{k} \\
& :=I_{k}+I I_{k}+I I I_{k} .
\end{aligned}
$$

Green's formula for the Lamé system and 4.48 yield

$$
\begin{aligned}
& \frac{\sqrt{\epsilon}}{m_{1}} I_{1}=\int_{D_{1} \cup D_{2}} \mathcal{L}_{\lambda, \mu} \mathbf{q}_{1} \cdot \mathbf{H}_{\Omega} \\
& =\left(\mathbf{H}_{\Omega}\left(\mathbf{p}_{1}\right)-\mathbf{H}_{\Omega}\left(\mathbf{p}_{2}\right)\right) \cdot \mathbf{e}_{1}-\sum_{j=1}^{2} \frac{\alpha_{2} a}{\left(\alpha_{1}-\alpha_{2}\right)}\left(\partial_{1} \mathbf{H}_{\Omega}\left(\mathbf{p}_{j}\right) \cdot \mathbf{e}_{1}+\partial_{2} \mathbf{H}_{\Omega}\left(\mathbf{p}_{j}\right) \cdot \mathbf{e}_{2}\right) .
\end{aligned}
$$

Since $a \approx \sqrt{\epsilon}$ and 4.69 holds, we have

$$
\sum_{j=1}^{2} \frac{\alpha_{2} a}{\left(\alpha_{1}-\alpha_{2}\right)}\left(\partial_{1} \mathbf{H}_{\Omega}\left(\mathbf{p}_{j}\right) \cdot \mathbf{e}_{1}+\partial_{2} \mathbf{H}_{\Omega}\left(\mathbf{p}_{j}\right) \cdot \mathbf{e}_{2}\right)=O(\sqrt{\epsilon}) .
$$

Since $\mathbf{p}_{1}=(-a, 0)$ and $\mathbf{p}_{2}=(a, 0)$, the mean value theorem shows that there is a point, say $\mathbf{p}_{*}$, on the line segment $\overline{\mathbf{p}_{1} \mathbf{p}_{2}}$ such that

$$
\left|\left(\mathbf{H}_{\Omega}\left(\mathbf{p}_{1}\right)-\mathbf{H}_{\Omega}\left(\mathbf{p}_{2}\right)\right) \cdot \mathbf{e}_{1}\right| \leq 2 a\left|\partial_{1} \mathbf{H}_{\Omega}\left(\mathbf{p}_{*}\right) \cdot \mathbf{e}_{1}\right| .
$$

So, we have

$$
\left|\left(\mathbf{H}_{\Omega}\left(\mathbf{p}_{1}\right)-\mathbf{H}_{\Omega}\left(\mathbf{p}_{2}\right)\right) \cdot \mathbf{e}_{1}\right| \lesssim \sqrt{\epsilon} .
$$

Therefore, from (4.71), we obtain

$$
I_{1}=O(1)
$$

Similarly, one can show

$$
I_{2}=O(1) .
$$

Next we estimate $I I_{k}$. Let $\mathbf{v} \in \mathcal{A}^{*}$ be the solution to the following exterior Dirichlet problem

$$
\begin{cases}\mathcal{L}_{\lambda, \mu} \mathbf{v}=0 & \text { in } D^{e} \\ \mathbf{v}=\mathbf{H}_{\Omega} & \text { on } \partial D^{e}\end{cases}
$$

From 2.37), we have

$$
\mathcal{E}_{D^{e}}[\mathbf{v}]=\min _{\mathbf{w} \in W} \mathcal{E}_{D^{e}}[\mathbf{w}]
$$

where

$$
W:=\left\{\mathbf{w} \in \mathcal{A}^{*}:\left.\mathbf{w}\right|_{\partial D^{e}}=\mathbf{H}_{\Omega}\right\} .
$$


Let $\mathbf{w}$ be a function such that

$$
\left\{\begin{array}{l}
\left.\mathbf{w}\right|_{\Pi_{L} \cup \partial D^{e}}=\mathbf{H}_{\Omega}, \\
\left.\mathbf{w}\right|_{\mathbb{R}^{2} \backslash B_{0}}=0, \\
\|\mathbf{w}\|_{H^{1}\left(\mathbb{R}^{2}\right)} \lesssim\|\mathbf{g}\|_{C^{1, \gamma}(\partial \Omega)},
\end{array}\right.
$$

where $B_{0}$ is a disk which contains $\overline{D_{1} \cup D_{2}}$. It is worth to mention that the third condition in the above is fulfilled thanks to 4.69 . Since $\mathbf{w} \in W$, we have

$$
\mathcal{E}_{D^{e}}[\mathbf{v}] \leq \mathcal{E}_{D^{e}}[\mathbf{w}] \lesssim 1
$$

Then, using Lemma 2.8 and Proposition 3.13 , we obtain

$$
\begin{aligned}
\left|I I_{k}\right| & =\left|\int_{\partial D^{e}} \partial_{\nu} \mathbf{r}_{k} \cdot \mathbf{v}\right| \\
& =\left|\int_{D^{e}} \mathbb{C} \widehat{\nabla} \mathbf{r}_{k}: \widehat{\nabla} \mathbf{v}\right| \lesssim \mathcal{E}_{D^{e}}\left[\mathbf{r}_{k}\right]^{1 / 2} \mathcal{E}_{D^{e}}[\mathbf{v}]^{1 / 2} \lesssim 1, \quad k=1,2 .
\end{aligned}
$$

Let us now consider $I I I_{k}$. We see from Lemma 3.6 and 3.8 that

$$
\left|\mathbf{r}_{k}\right|_{\partial D_{i}}|=| \frac{(-1)^{i}}{2} \Psi_{k}-\left.\frac{m_{k}}{\sqrt{\epsilon}} \mathbf{q}_{k}\right|_{\partial D_{i}} \mid \lesssim 1
$$

So it follows from 4.70 that

$$
\left|I I I_{k}\right| \lesssim\left\|\partial_{\nu} \mathbf{H}_{\Omega}\right\|_{L^{2}(\partial \Omega)} \lesssim 1, \quad k=1,2
$$

Therefore, we have

$$
\left|\mathcal{J}_{\Omega, k}\right| \leq\left|I_{k}\right|+\left|I I_{k}\right|+\left|I I I_{k}\right| \lesssim 1, \quad k=1,2 .
$$

To deal with the case when $k=3$, let $\mathbf{v}$ be the solution to 4.72 as before. It follows from 4.59) and 4.75 that

$$
\begin{aligned}
\left|\mathcal{J}_{\Omega, 3}\right| & =\left|\int_{\partial D^{e}} \partial_{\nu} \mathbf{h}_{3} \cdot \mathbf{v}\right| \\
& =\left|\int_{D^{e}} \mathbb{C} \widehat{\nabla} \mathbf{h}_{3}: \widehat{\nabla} \mathbf{v}\right| \lesssim \mathcal{I}_{33}^{1 / 2} \mathcal{E}_{D^{e}}[\mathbf{v}]^{1 / 2} \lesssim 1 .
\end{aligned}
$$

The proof is completed.

Proposition 4.2 follows from Lemma 4.11, 4.12 and 4.13 .

\subsection{Proof of Proposition 4.3}

Set

$$
\mathcal{I}:=\left[\begin{array}{lll}
\mathcal{I}_{11} & \mathcal{I}_{12} & \mathcal{I}_{13} \\
\mathcal{I}_{12} & \mathcal{I}_{22} & \mathcal{I}_{23} \\
\mathcal{I}_{13} & \mathcal{I}_{23} & \mathcal{I}_{33}
\end{array}\right]
$$


From Lemma 4.11 and 4.12 , we have

$$
\begin{aligned}
\operatorname{det} \mathcal{I} & =\mathcal{I}_{11}\left(\mathcal{I}_{22} \mathcal{I}_{33}-\mathcal{I}_{23}^{2}\right)-\mathcal{I}_{12}\left(\mathcal{I}_{12} \mathcal{I}_{33}-\mathcal{I}_{13} \mathcal{I}_{23}\right)+\mathcal{I}_{13}\left(\mathcal{I}_{12} \mathcal{I}_{23}-\mathcal{I}_{13} \mathcal{I}_{22}\right) \\
& =\mathcal{I}_{11} \mathcal{I}_{22} \mathcal{I}_{33}+O\left(\epsilon^{-1 / 2}\right)=\frac{m_{1} m_{2}}{\epsilon} \mathcal{I}_{33}(1+O(\sqrt{\epsilon})) .
\end{aligned}
$$

So, by 4.59), the matrix $\mathcal{I}$ is invertible for sufficiently small $\epsilon$.

By Lemma 4.1 and Cramer's rule, we have

$$
\begin{aligned}
c_{11}-c_{21}= & \frac{\mathcal{J}_{\Omega, 1}}{\operatorname{det} \mathcal{I}}\left(\mathcal{I}_{22} \mathcal{I}_{33}-\mathcal{I}_{23}^{2}\right)-\frac{\mathcal{J}_{\Omega, 2}}{\operatorname{det} \mathcal{I}}\left(\mathcal{I}_{12} \mathcal{I}_{33}-\mathcal{I}_{13} \mathcal{I}_{23}\right) \\
& +\frac{\mathcal{J}_{\Omega, 3}}{\operatorname{det} \mathcal{I}}\left(\mathcal{I}_{12} \mathcal{I}_{23}-\mathcal{I}_{13} \mathcal{I}_{22}\right) .
\end{aligned}
$$

Recall from Lemma $4.11,4.12$ and 4.13 that

$$
\mathcal{I}_{11}, \mathcal{I}_{22} \approx \epsilon^{-1 / 2}, \quad\left|\mathcal{I}_{12}\right| \lesssim 1+\tau|\ln \epsilon|, \quad\left|\mathcal{I}_{j 3}\right| \lesssim 1, \quad \mathcal{I}_{33} \approx 1
$$

and

$$
\left|\mathcal{J}_{\Omega, j}\right| \lesssim\|\mathbf{g}\|_{C^{1, \gamma}(\partial \Omega)}
$$

for $j=1,2,3$. For simplicity, we may assume $\|\mathbf{g}\|_{C^{1, \gamma}(\partial \Omega)}=1$. Then, from (4.77) and (4.78), we can easily check that

$$
c_{11}-c_{21}=\frac{\mathcal{J}_{\Omega, 1}}{\operatorname{det} \mathcal{I}} \mathcal{I}_{22} \mathcal{I}_{33}-\frac{\mathcal{J}_{\Omega, 3}}{\operatorname{det} \mathcal{I}} \mathcal{I}_{13} \mathcal{I}_{22}+O(\epsilon+\tau \epsilon|\ln \epsilon|)
$$

Hence, by applying 4.55) and the second equality in (4.77), we obtain

$$
c_{11}-c_{21}=\frac{\sqrt{\epsilon}}{m_{1}}\left(\mathcal{J}_{\Omega, 1}-\frac{\mathcal{J}_{\Omega, 3} \mathcal{I}_{13}}{\mathcal{I}_{33}}\right)+O(\epsilon+\tau \epsilon|\ln \epsilon|)
$$

Similarly, we have

$$
\begin{aligned}
& c_{12}-c_{22}=\frac{\sqrt{\epsilon}}{m_{2}}\left(\mathcal{J}_{\Omega, 2}-\frac{\mathcal{J}_{\Omega, 3} \mathcal{I}_{23}}{\mathcal{I}_{33}}\right)+O(\epsilon+\tau \epsilon|\ln \epsilon|), \\
& c_{13}-c_{23}=\frac{\mathcal{J}_{\Omega, 3}}{\mathcal{I}_{33}}+O(\sqrt{\epsilon}+\tau \sqrt{\epsilon}|\ln \epsilon|) .
\end{aligned}
$$

Finally, the definition (4.7) of $\mathcal{K}_{\Omega, j}$ yield (4.9)-(4.11). The proof of Proposition 4.3 is completed.

\subsection{Proof of Proposition 4.5}

To prove 4.16 we modify the function $\mathbf{r}_{j}^{K}$ introduced in 3.86 . Let $\mathbf{r}_{\Omega, j}^{K}$ be a function in $C^{2}\left(\mathbb{R}^{2}\right)$ such that

$$
\begin{cases}\mathbf{r}_{\Omega, j}^{K}=\left.\mathbf{r}_{j}^{K}\right|_{\Pi_{L_{0}}} & \text { in } \Pi_{L_{0}}, \\ \mathbf{r}_{\Omega, j}^{K}=\frac{(-1)^{i}}{2} \Psi_{j}-\frac{m_{j}}{\sqrt{\epsilon}} \mathbf{q}_{j} & \text { on } \partial D_{i}, i=1,2, \\ \mathbf{r}_{\Omega, j}^{K}=-\frac{m_{j}}{\sqrt{\epsilon}} \mathbf{q}_{j} & \text { on } \partial \Omega,\end{cases}
$$


and

$$
\left\|\mathbf{r}_{\Omega, j}^{K}\right\|_{H^{1}\left(\mathbb{R}^{2} \backslash \Pi_{L_{0}}\right)} \lesssim 1
$$

We emphasize that $\mathbf{r}_{\Omega, j}^{K}=\mathbf{r}_{j}^{K}$ is a linear interpolation in the gap region $\Pi_{L_{0}}$. Note that $\left.\nabla \mathbf{r}_{j}^{K}\right|_{\Pi_{L_{0}}}$ is already estimated in Lemma 3.14 . Let

$$
\mathbf{w}_{j}:=\mathbf{r}_{\Omega, j}-\mathbf{r}_{\Omega, j}^{K}, \quad j=1,2,
$$

where $\mathbf{r}_{\Omega, j}$ is the function defined by 4.15 . We see that the function $\mathbf{w}_{j}$ is the solution to the following problem:

$$
\begin{cases}\mathcal{L}_{\lambda, \mu} \mathbf{w}_{j}=-\mathcal{L}_{\lambda, \mu} \mathbf{r}_{\Omega, j}^{K} & \text { in } \widetilde{\Omega}, \\ \mathbf{w}_{j}=0 & \text { on } \partial D_{1} \cup \partial D_{2} \cup \partial \Omega .\end{cases}
$$

The following lemma can be proved by arguments parallel to the proof of Lemma 3.6 in [9]. So, we omit the proof.

Lemma 4.14. Let $\mathbf{v}$ be a solution to $\mathcal{L}_{\lambda, \mu} \mathbf{v}=-\mathcal{L}_{\lambda, \mu} \mathbf{f}$ in $\widetilde{\Omega}$ with $\mathbf{v}=0$ on $\partial D_{1} \cup \partial D_{2} \cup \partial \Omega$, where $\mathbf{f}$ is a given function belongs to $C^{2}\left(\mathbb{R}^{2}\right)$. Assume that the following conditions hold:

(i) The function $\mathbf{v}$ satisfies

$$
\int_{\widetilde{\Omega}}|\nabla \mathbf{v}|^{2} \lesssim 1
$$

(ii) The function $\mathbf{f}$ satisfies

$$
\left|\left(\mathcal{L}_{\lambda, \mu} \mathbf{f}\right)(x, y)\right| \lesssim \frac{1}{\epsilon+y^{2}} \quad \text { for }(x, y) \in \Pi_{L}
$$

Then we have, for $0<L^{\prime}<L$,

$$
\|\nabla \mathbf{v}\|_{L^{\infty}\left(\Pi_{L^{\prime}}\right)} \lesssim 1
$$

Lemma 4.15. For $j=1,2$, let $\mathbf{w}_{j}$ be the solution to (4.82). Then we have

$$
\left|\nabla \mathbf{w}_{j}(\mathbf{x})\right| \lesssim 1 \quad \text { for } \mathbf{x} \in \Pi_{L_{0}} .
$$

Proof. It suffices to show that the hypotheses (i) and (ii) of Lemma 4.14 are fulfilled, namely,

$$
\int_{\widetilde{\Omega}}\left|\nabla \mathbf{w}_{j}\right|^{2} \lesssim 1
$$

and

$$
\left|\left(\mathcal{L}_{\lambda, \mu} \mathbf{r}_{\Omega, j}^{K}\right)(x, y)\right| \lesssim \frac{1}{\epsilon+y^{2}} \quad \text { for }(x, y) \in \Pi_{L}
$$


By the first Korn's inequality, the variational principle and Lemma 3.14, we have

$$
\begin{aligned}
\int_{\widetilde{\Omega}}\left|\nabla \mathbf{w}_{j}\right|^{2} & \leq 2 \int_{\widetilde{\Omega}}\left|\widehat{\nabla} \mathbf{w}_{j}\right|^{2} \lesssim \int_{\widetilde{\Omega}} \mathbb{C} \widehat{\nabla} \mathbf{w}_{j}: \widehat{\nabla} \mathbf{w}_{j} \\
& \lesssim \int_{\widetilde{\Omega}} \mathbb{C} \widehat{\nabla} \mathbf{r}_{\Omega, j}: \widehat{\nabla} \mathbf{r}_{\Omega, j}+\int_{\widetilde{\Omega}} \mathbb{C} \widehat{\nabla} \mathbf{r}_{\Omega, j}^{K}: \widehat{\nabla} \mathbf{r}_{\Omega, j}^{K} \\
& \lesssim \int_{\widetilde{\Omega}} \mathbb{C} \widehat{\nabla} \mathbf{r}_{\Omega, j}^{K}: \widehat{\nabla} \mathbf{r}_{\Omega, j}^{K} \\
& \lesssim \int_{\Pi_{L_{0}}}\left|\nabla \mathbf{r}_{j}^{K}\right|^{2}+\int_{\widetilde{\Omega} \backslash \Pi_{L_{0}}}\left|\nabla \mathbf{r}_{j}^{K}\right|^{2} \lesssim \int_{-L_{0}}^{L_{0}} \int_{-f_{1}(y)}^{f_{2}(y)} \frac{\epsilon+|y|}{\epsilon+y^{2}} d x d y+1 \\
& \lesssim \int_{-L_{0}}^{L_{0}}(\epsilon+|y|) d y+1 \lesssim 1
\end{aligned}
$$

So we obtain (4.84).

We now prove (4.85). Let $d, \phi$ and $\eta$ be the function defined in (3.89). It follows from (3.90) and (3.91) that, for $(x, y) \in \Pi_{L_{0}}$,

$$
\begin{aligned}
\partial_{11} r_{\Omega, 11}^{K}=\partial_{11} r_{11}^{K}= & 0, \\
\partial_{12} r_{\Omega, 11}^{K}=\partial_{12} r_{11}^{K}= & \frac{\phi^{\prime}}{d}-\frac{\phi d^{\prime}}{d^{2}}, \\
\partial_{22} r_{\Omega, 11}^{K}=\partial_{22} r_{11}^{K}= & {\left[\frac{\phi^{\prime \prime}}{d}-\frac{2 \phi^{\prime} d^{\prime}}{d^{2}}-\frac{\phi d^{\prime \prime}}{d^{2}}+\frac{2 \phi d^{\prime 2}}{d^{3}}\right] x } \\
& +\frac{\phi^{\prime \prime} f_{1}}{d}+2 \frac{\phi^{\prime} f_{1}^{\prime}}{d}-2 \frac{\phi^{\prime} f_{1} d^{\prime}}{d^{2}}+\frac{\phi f_{1}^{\prime \prime}}{d} \\
& -2 \frac{\phi f_{1}^{\prime} d^{\prime}}{d^{2}}-\frac{\phi f_{1} d^{\prime \prime}}{d^{2}}+2 \frac{\phi f_{1} d^{\prime 2}}{d^{3}}+\eta^{\prime \prime} .
\end{aligned}
$$

In addition to $(3.92)$ and $(3.93)$, we have

$$
\left|\phi^{\prime \prime}(y)\right|,\left|\eta^{\prime \prime}(y)\right| \lesssim \frac{1}{\epsilon+y^{2}}
$$

Then, using (3.92), 3.93, 4.86, 4.87) and the fact that $|x| \lesssim \epsilon+y^{2}$ for $(x, y) \in \Pi_{L_{0}}$, we have

$$
\left|\partial_{12} r_{\Omega, 11}^{K}\right| \lesssim \frac{1}{\epsilon+y^{2}}+\frac{\left(\epsilon+y^{2}\right)|y|}{\left(\epsilon+y^{2}\right)^{2}} \lesssim \frac{1}{\epsilon+y^{2}}
$$

and

$$
\begin{aligned}
\left|\partial_{22} r_{\Omega, 11}^{K}\right| \lesssim[ & \left.\frac{1}{\epsilon+y^{2}}+\frac{|y|}{\left(\epsilon+y^{2}\right)^{2}}+\frac{\epsilon+y^{2}}{\left(\epsilon+y^{2}\right)^{2}}+\frac{\left(\epsilon+y^{2}\right) y^{2}}{\left(\epsilon+y^{2}\right)^{3}}\right]\left(\epsilon+y^{2}\right) \\
& +\frac{1}{\epsilon+y^{2}} \frac{\epsilon+y^{2}}{\epsilon+y^{2}}+\frac{|y|}{\epsilon+y^{2}}+\frac{\left(\epsilon+y^{2}\right)|y|}{\left(\epsilon+y^{2}\right)^{2}}+\frac{\epsilon+y^{2}}{\epsilon+y^{2}} \\
& +\frac{\left(\epsilon+y^{2}\right) y^{2}}{\left(\epsilon+y^{2}\right)^{2}}+\frac{\left(\epsilon+y^{2}\right)\left(\epsilon+y^{2}\right)}{\left(\epsilon+y^{2}\right)^{2}}+\frac{\left(\epsilon+y^{2}\right)\left(\epsilon+y^{2}\right) y^{2}}{\left(\epsilon+y^{2}\right)^{3}}+\frac{1}{\epsilon+y^{2}} \\
\lesssim & \frac{1}{\epsilon+y^{2}} .
\end{aligned}
$$


The proof is completed.

Now we are ready to prove Proposition 4.5.

Proof of Proposition 4.5. Let us look into estimates in $\widetilde{\Omega} \backslash \Pi_{L}$ first. Let $\mathbf{v}_{i j}$ be the function defined in 4.29 . It is proved in [9] that

$$
\left\|\nabla \mathbf{v}_{i j}\right\|_{L^{\infty}\left(\widetilde{\Omega} \backslash \Pi_{L}\right)} \lesssim 1, \quad i=1,2, \quad j=1,2,3 .
$$

Since $\mathbf{h}_{\Omega, j}=-\frac{1}{2} \mathbf{v}_{1 j}+\frac{1}{2} \mathbf{v}_{2 j}$, we have

$$
\left\|\nabla \mathbf{h}_{\Omega, j}\right\|_{L^{\infty}\left(\widetilde{\Omega} \backslash \Pi_{L_{0}}\right)} \lesssim 1, \quad j=1,2,3 .
$$

This estimate together with $(3.58)$ and $(3.80)$ yields the second part of $(4.16)$ and 4.18 on $\widetilde{\Omega} \backslash \Pi_{L_{0}}$.

By (3.88) and the first line of (4.79), we have

$$
\left|\nabla \mathbf{r}_{\Omega, j}^{K}(\mathbf{x})\right|=\left|\nabla \mathbf{r}_{j}^{K}(\mathbf{x})\right| \lesssim 1+\frac{\tau|y|}{\epsilon+y^{2}} \quad \text { for } \mathbf{x} \in \Pi_{L_{0}}
$$

Then, the first part of (4.16) follows from 4.81) and (4.83).

The estimate 4.18) on $\Pi_{L}$ follows from 4.30, 4.33), 4.61) and 4.65). In fact, we have on $\Pi_{L}$

$$
\begin{aligned}
\nabla \mathbf{h}_{\Omega, 3} & =-\frac{1}{2} \nabla \mathbf{v}_{13}+\frac{1}{2} \nabla \mathbf{v}_{23} \\
& =-\frac{1}{2} \nabla \mathbf{v}_{13}^{K}+\frac{1}{2} \nabla \mathbf{v}_{23}^{K}+O(1) \\
& =\nabla \mathbf{h}_{3}^{K}+O(1)=\nabla \mathbf{q}_{3}+O(1) .
\end{aligned}
$$

This completes the proof.

\section{Stress concentration-the free space problem}

In this section we consider the free space problem 2.10 and characterize the singular behavior of its solution.

Analogously to $\mathcal{J}_{\Omega, j}$ in 4.5 , we define

$$
\mathcal{J}_{j}=\int_{\partial D^{e}} \partial_{\nu} \mathbf{h}_{j} \cdot \mathbf{H}, \quad j=1,2,3,
$$

where $\mathbf{H}$ is the background solution of the problem (2.10). It is worth emphasizing that $\mathcal{J}_{j}$ is defined using $\mathbf{H}$ while $\mathcal{J}_{\Omega, j}$ uses $\mathbf{H}_{\Omega}$. Analogously to $\mathcal{K}_{\Omega, j}$ in (4.7), we define

$$
\mathcal{K}_{1}=\mathcal{J}_{1}-\frac{\mathcal{J}_{3} \mathcal{I}_{13}}{\mathcal{I}_{33}}, \quad \mathcal{K}_{2}=\mathcal{J}_{2}-\frac{\mathcal{J}_{3} \mathcal{I}_{23}}{\mathcal{I}_{33}}, \quad \mathcal{K}_{3}=\frac{\mathcal{J}_{3}}{\mathcal{I}_{33}}
$$

Then the constants $\mathcal{K}_{j}$ are bounded regardless of $\epsilon$ (see (5.11)).

The following is the main result of this section 
Theorem 5.1. Let $\mathbf{u}$ be the solution to (2.10). Then we have the following decomposition of $\mathbf{u}-\mathbf{H}$ :

$$
(\mathbf{u}-\mathbf{H})(\mathbf{x})=\mathbf{b}(\mathbf{x})-\sum_{j=1}^{3}\left(\mathcal{K}_{j}+s_{j}\right) \mathbf{q}_{j}(\mathbf{x}), \quad \mathbf{x} \in D^{e},
$$

where the constants $s_{j}, j=1,2,3$, satisfy

$$
\left|s_{j}\right| \lesssim \tau \sqrt{\epsilon}|\ln \epsilon|\|\mathbf{H}\|_{H^{1}(B)},
$$

and the function $\mathbf{b}$ satisfies

$$
\|\nabla \mathbf{b}\|_{L^{\infty}\left(D^{e}\right)} \lesssim\|\mathbf{H}\|_{H^{1}(B)}
$$

Here, $B$ is a disk containing $\overline{D_{1} \cup D_{2}}$.

By the proof analogous to that of Theorem 4.7, we can derive the following theorem from Theorem 5.1.

Theorem 5.2. It holds that

$$
\frac{\sum_{j=1,2}\left|\mathcal{K}_{j}\right|}{\sqrt{\epsilon}} \lesssim\|\nabla(\mathbf{u}-\mathbf{H})\|_{L^{\infty}\left(D^{e}\right)} \lesssim \frac{\|\mathbf{H}\|_{H^{1}(B)}}{\sqrt{\epsilon}} .
$$

We prove Theorem 5.1 based on Theorem 4.6 . Let $B$ be a disk containing $\overline{D_{1} \cup D_{2}}$. We assume for convenience that the center of $B$ is 0 . Then the solution $\mathbf{u}$ to $(2.10)$ is the solution to 2.6$)$ with $\Omega=B$ and $\mathbf{g}=\left.\mathbf{u}\right|_{\partial B}$. So we obtain the following decomposition of the solution $\mathbf{u}$ in $B$ by applying Theorem 4.6 .

$$
\mathbf{u}=\mathbf{b}_{B}-\sum_{j=1}^{3}\left(\mathcal{K}_{B, j}+s_{B, j}\right) \mathbf{q}_{j} \quad \text { in } D^{e} \cap B,
$$

where the constants $s_{B, j}$ and the function $\mathbf{b}_{B}$ satisfy

$$
\left|s_{B, j}\right| \lesssim \tau \sqrt{\epsilon}|\ln \epsilon|\|\mathbf{u}\|_{C^{1, \gamma}(\partial B)}
$$

and

$$
\left\|\nabla \mathbf{b}_{B}\right\|_{L^{\infty}\left(D^{e} \cap B\right)} \lesssim\|\mathbf{u}\|_{C^{1, \gamma}(\partial B)},
$$

Although (5.7) looks similar to (5.3), there are three things to be clarified. First, the coefficient of $\mathbf{q}_{j}$ in (5.7) is given by $\mathcal{K}_{B, j}$, not by $\mathcal{K}_{j}$. Second, the right-hand sides of (5.8) and (5.9) depend on $\epsilon$ since $\|\mathbf{u}\|_{C^{1, \gamma}(\partial B)}$ does. We need to prove the $\|\mathbf{u}\|_{C^{1, \gamma}(\partial B)}$ is bounded regardless of $\epsilon$. Third, the decomposition is valid only in $B$, not in the whole region $D^{e}$. In the following we elaborate on these issues to show that (5.7)-(5.9) actually yield (5.3)-(5.5).

Lemma 5.3. $\mathcal{K}_{B, j}=\mathcal{K}_{j}$ for $j=1,2,3$. 
Proof. By Green's formula and the fact that $\mathbf{u}-\mathbf{H} \in \mathcal{A}$, we have

$$
-\mathcal{S}_{\partial B}\left[\left.\partial_{\nu}(\mathbf{u}-\mathbf{H})\right|_{\partial B}\right](\mathbf{x})+\mathcal{D}_{\partial B}\left[\left.(\mathbf{u}-\mathbf{H})\right|_{\partial B}\right](\mathbf{x})=0, \quad \mathbf{x} \in B .
$$

Then, by Green's formula again, we have

$$
\begin{aligned}
\mathbf{H}_{B}(\mathbf{x}) & =-\mathcal{S}_{\partial B}\left[\left.\partial_{\nu} \mathbf{u}\right|_{\partial B}\right](\mathbf{x})+\mathcal{D}_{\partial B}\left[\left.\mathbf{u}\right|_{\partial B}\right](\mathbf{x}) \\
& =-\mathcal{S}_{\partial B}\left[\left.\partial_{\nu} \mathbf{H}\right|_{\partial B}\right](\mathbf{x})+\mathcal{D}_{\partial B}\left[\left.\mathbf{H}\right|_{\partial B}\right](\mathbf{x})=\mathbf{H}(\mathbf{x}),
\end{aligned}
$$

for $\mathbf{x} \in B$. Therefore, Lemma 5.3 follows from (4.5), (4.7) and (5.2).

Lemma 5.4. Let $B_{1}$ be a disk containing $\bar{B}$. We have

$$
\|\mathbf{u}\|_{C^{1, \gamma}(\partial B)} \lesssim\|\mathbf{H}\|_{H^{1}\left(B_{1}\right)}
$$

and

$$
\left|\mathcal{K}_{j}\right| \lesssim\|\mathbf{H}\|_{H^{1}\left(B_{1}\right)}, \quad j=1,2,3 .
$$

Proof. By Proposition 2.10, we have

$$
\|\mathbf{u}\|_{C^{1, \gamma}(\partial B)} \leq\|\mathbf{u}-\mathbf{H}\|_{C^{1, \gamma}(\partial B)}+\|\mathbf{H}\|_{C^{1, \gamma}(\partial B)} \lesssim\|\mathbf{H}\|_{H^{1}\left(B_{1}\right)} .
$$

By Proposition 4.2 and Lemma 5.3 , we have

$$
\left|\mathcal{K}_{j}\right|=\left|\mathcal{K}_{B, j}\right| \lesssim\|\mathbf{u}\|_{C^{1, \gamma}(\partial B)}
$$

So, (5.11) follows from (5.10).

Proof of Theorem 5.1. Let $s_{j}:=s_{B, j}$. Then it follows from (5.8) and 5.10 that

$$
\left|s_{j}\right| \lesssim \tau \sqrt{\epsilon}|\ln \epsilon|\|\mathbf{H}\|_{H^{1}\left(B_{1}\right)}
$$

Let

$$
\mathbf{b}:=\mathbf{u}-\mathbf{H}+\sum_{j=1}^{3}\left(\mathcal{K}_{j}+s_{j}\right) \mathbf{q}_{j}
$$

To estimate $\mathbf{b}$ in $D^{e}$, we split the region $D^{e}$ into $D^{e} \cap B$ and $D^{e} \backslash B$. Using (5.7), Lemma 5.3 and the fact that $s_{j}=s_{B, j}$, we have

$$
\mathbf{b}=\left(\mathbf{u}+\sum_{j=1}^{3}\left(\mathcal{K}_{B, j}+s_{B, j}\right) \mathbf{q}_{j}\right)-\mathbf{H}=\mathbf{b}_{B}-\mathbf{H} \quad \text { in } D^{e} \cap B .
$$

So, we infer from $(5.9)$ and $(5.10)$ that

$$
\begin{aligned}
\|\nabla \mathbf{b}\|_{L^{\infty}\left(D^{e} \cap B\right)} & =\left\|\nabla \mathbf{b}_{B}\right\|_{L^{\infty}\left(D^{e} \cap B\right)}+\|\nabla \mathbf{H}\|_{L^{\infty}\left(D^{e} \cap B\right)} \\
& \lesssim\|\mathbf{u}\|_{C^{1, \gamma}(\partial B)}+\|\nabla \mathbf{H}\|_{L^{\infty}\left(D^{e} \cap B\right)} \lesssim\|\mathbf{H}\|_{H^{1}\left(B_{1}\right)}
\end{aligned}
$$


Let us now consider estimates on the region $D^{e} \backslash B$. From Proposition 2.10 and (5.13), we see that

$$
\begin{aligned}
\|\nabla \mathbf{b}\|_{L^{\infty}\left(D^{e} \backslash B\right)} & =\left\|\nabla(\mathbf{u}-\mathbf{H})+\sum_{j=1}^{3}\left(\mathcal{K}_{j}+s_{j}\right) \nabla \mathbf{q}_{j}\right\|_{L^{\infty}\left(D^{e} \backslash B\right)} \\
& \leq\|\nabla(\mathbf{u}-\mathbf{H})\|_{L^{\infty}\left(D^{e} \backslash B\right)}+\left\|\sum_{j=1}^{3}\left(\mathcal{K}_{j}+s_{j}\right) \nabla \mathbf{q}_{j}\right\|_{L^{\infty}\left(D^{e} \backslash B\right)} \\
& \leq\|\mathbf{H}\|_{H^{1}\left(B_{1}\right)}+\left\|\sum_{j=1}^{3}\left(\mathcal{K}_{j}+s_{j}\right) \nabla \mathbf{q}_{j}\right\|_{L^{\infty}\left(D^{e} \backslash B\right)}
\end{aligned}
$$

Lemma 3.6 and 3.80 show that

$$
\left\|\nabla \mathbf{q}_{j}\right\|_{L^{\infty}\left(D^{e} \backslash B\right)} \lesssim 1, \quad j=1,2,3 .
$$

Therefore, from (5.11) and (5.12), we obtain

$$
\|\nabla \mathbf{b}\|_{L^{\infty}\left(D^{e} \backslash B\right)} \leq\|\mathbf{H}\|_{H^{1}\left(B_{1}\right)}+(1+\sqrt{\epsilon}|\ln \epsilon|)\|\mathbf{H}\|_{H^{1}\left(B_{1}\right)} \leq\|\mathbf{H}\|_{H^{1}\left(B_{1}\right)} .
$$

So we have

$$
\|\nabla \mathbf{b}\|_{L^{\infty}\left(D^{e}\right)} \lesssim\|\mathbf{H}\|_{H^{1}\left(B_{1}\right)}
$$

and the proof of Theorem 5.1 is completed (with $B$ replaced by $B_{1}$ ).

\section{Symmetric inclusions and optimality of the blow-up rate}

In this section, we show that (5.3) can be further simplified by assuming some symmetry of the inclusions $D_{1}$ and $D_{2}$. More importantly, we show that the blow-up rate $\epsilon^{-1 / 2}$ of $|\nabla \mathbf{u}|$ is optimal by considering two circular inclusions. The singular functions $\mathbf{q}_{j}$ play an essential role here as well.

Let us first assume that the background field $\mathbf{H}$ can be decomposed as

$$
\mathbf{H}=\mathbf{H}_{e}+\mathbf{H}_{o},
$$

where $\mathbf{H}_{e}=\left(H_{e 1}, H_{e 2}\right)^{T}$ and $\mathbf{H}_{o}=\left(H_{o 1}, H_{o 2}\right)^{T}$ respectively have the following symmetric properties:

$$
\left\{\begin{array}{l}
H_{e 1}(x, y)=H_{e 1}(x,-y)=-H_{e 1}(-x, y), \\
H_{e 2}(x, y)=-H_{e 2}(x,-y)=H_{e 2}(-x, y),
\end{array}\right.
$$

and

$$
\left\{\begin{array}{l}
H_{o 1}(x, y)=-H_{o 1}(x,-y)=H_{o 1}(-x, y), \\
H_{o 2}(x, y)=H_{o 2}(x,-y)=-H_{o 2}(-x, y) .
\end{array}\right.
$$

If $\mathbf{H}$ is a uniform loading, that is, $\mathbf{H}(\mathbf{x})=(A x, B y)^{T}+C(y, x)^{T}$ for some real coefficients $A, B$ and $C$, then we may take $\mathbf{H}_{e}=(A x, B y)^{T}$ and $\mathbf{H}_{o}=C(y, x)^{T}$. 


\subsection{Symmetric inclusions}

Let us assume that $D_{1} \cup D_{2}$ is symmetric with respect to both $x$ - and $y$-axes. Then we have the following theorem.

Theorem 6.1. Let $\mathbf{u}$ be the solution to (2.10) under the assumption that $D_{1} \cup D_{2}$ is symmetric with respect to both $x$-and $y$-axes. Then, it holds that

$$
(\mathbf{u}-\mathbf{H})(\mathbf{x})=\mathbf{b}(\mathbf{x})+\mathcal{J}_{1} \mathbf{q}_{1}(\mathbf{x})+\mathcal{J}_{2} \mathbf{q}_{2}(\mathbf{x}), \quad \mathbf{x} \in D^{e},
$$

where the function $\mathbf{b}$ satisfies

$$
|\nabla \mathbf{b}(\mathbf{x})| \lesssim\|\mathbf{H}\|_{H^{1}(B)} \quad \text { for all } \mathbf{x} \in D^{e} .
$$

Here, $B$ is a disk containing $\overline{D_{1} \cup D_{2}}$. Moreover, if $\mathbf{H}=\mathbf{H}_{e}$, i.e., $\mathbf{H}$ satisfies (6.2), then $\mathcal{J}_{2}=0$; If $\mathbf{H}$ satisfies 6.3), then $\mathcal{J}_{1}=0$.

Proof. Since $D_{1}$ and $D_{2}$ are symmetric, the number $\tau$ defined by (2.54) is 0 . So it follows from (5.4) that $s_{j}=0$ for $j=1,2,3$.

Now it remains to show that $\mathcal{K}_{1}=\mathcal{J}_{1}, \mathcal{K}_{1}=\mathcal{J}_{2}$ and $\mathcal{K}_{3}=0$, for which it is enough to show that $\mathcal{J}_{3}=0$ by the definition $(5.2)$ of $\mathcal{K}_{j}$. Recall that

$$
\mathcal{J}_{3}=\int_{\partial D^{e}} \partial_{\nu} \mathbf{h}_{3} \cdot \mathbf{H}
$$

Let $\mathbf{h}_{3}=\left(h_{31}, h_{32}\right)^{T}$. Thanks to the symmetry of the inclusions and the boundary condition of $\mathbf{h}_{3}$, one can see that the following two functions are also solutions of (3.1):

$$
\left[\begin{array}{c}
-h_{31}(x,-y) \\
h_{32}(x,-y)
\end{array}\right], \quad\left[\begin{array}{c}
-h_{31}(-x, y) \\
h_{32}(-x, y)
\end{array}\right]
$$

So, by the uniqueness of the solution we see that $\mathbf{h}_{3}$ satisfies the following symmetry:

$$
\left\{\begin{array}{l}
h_{31}(x, y)=-h_{31}(x,-y)=-h_{31}(-x, y), \\
h_{32}(x, y)=h_{32}(x,-y)=h_{32}(-x, y) .
\end{array}\right.
$$

The outward normal $\mathbf{n}=\left(n_{1}, n_{2}\right)^{T}$ to $\partial D^{e}$ satisfies

$$
\left\{\begin{array}{l}
n_{1}(x, y)=n_{1}(x,-y)=-n_{1}(-x, y), \\
n_{2}(x, y)=-n_{2}(x,-y)=n_{2}(-x, y) .
\end{array}\right.
$$

So, the conormal derivative $\mathbf{f}:=\partial_{\nu} \mathbf{h}_{3}$ on $\partial D^{e}$ enjoys the following symmetry:

$$
\left\{\begin{array}{l}
f_{1}(x, y)=-f_{1}(x,-y)=-f_{1}(-x, y), \\
f_{2}(x, y)=f_{2}(x,-y)=f_{2}(-x, y) .
\end{array}\right.
$$

Let $\mathbf{H}=\mathbf{H}_{e}+\mathbf{H}_{o}$ be the decomposition as in 6.1). We write $\mathcal{J}_{3}$ as

$$
\mathcal{J}_{3}=\int_{\partial D^{e}} \mathbf{f} \cdot \mathbf{H}_{e}+\int_{\partial D^{e}} \mathbf{f} \cdot \mathbf{H}_{o}=: I+I I .
$$


Using the symmetry with respect to the $y$-axis in $(6.2)$ and $(6.8)$, we have

$$
\begin{aligned}
I & =\int_{\partial D_{1}}\left(f_{1}, f_{2}\right) \cdot\left(H_{e 1}, H_{e 2}\right)+\int_{\partial D_{2}}\left(f_{1}, f_{2}\right) \cdot\left(H_{e 1}, H_{e 2}\right) \\
& =\int_{\partial D_{1}}\left(f_{1}, f_{2}\right) \cdot\left(H_{e 1}, H_{e 2}\right)+\int_{\partial D_{1}}\left(-f_{1}, f_{2}\right) \cdot\left(-H_{e 1}, H_{e 2}\right) \\
& =2 \int_{\partial D_{1}} f_{1} H_{e 1}+f_{2} H_{e 2} .
\end{aligned}
$$

Then, the symmetry with respect to the $x$-axis in 6.2 and $(6.8)$, we obtain

$$
\begin{aligned}
I & =2 \int_{\partial D_{1} \cap\{y \geq 0\}}\left(f_{1} H_{e 1}+f_{2} H_{e 2}\right)+2 \int_{\partial D_{1} \cap\{y<0\}}\left(f_{1} H_{e 1}+f_{2} H_{e 2}\right) \\
& =2 \int_{\partial D_{1} \cap\{y \geq 0\}}\left(f_{1} H_{e 1}+f_{2} H_{e 2}\right)+2 \int_{\partial D_{1} \cap\{y \geq 0\}}\left(\left(-f_{1}\right) H_{e 1}+f_{2}\left(-H_{e 2}\right)\right)=0 .
\end{aligned}
$$

By the exactly same way, we can show $I I=0$.

Suppose that $\mathbf{H}$ has the symmetry property (6.2). Let $\mathbf{g}:=\partial_{\nu} \mathbf{h}_{2}$. Then (3.7) and (6.7) show that $\mathbf{g}$ has the following symmetry properties:

$$
\left\{\begin{array}{l}
g_{1}(x, y)=-g_{1}(x,-y)=g_{1}(-x, y) \\
g_{2}(x, y)=g_{2}(x,-y)=-g_{2}(-x, y)
\end{array}\right.
$$

So one can see as before that

$$
\mathcal{J}_{2}=\int_{\partial D^{e}} \mathbf{g} \cdot \mathbf{H}_{e}=0
$$

Similarly, one can show that $\mathcal{J}_{1}=0$ if $\mathbf{H}$ has the symmetry property (6.3). This completes the proof.

Corollary 6.2. Under the same hypothesis as in Theorem 6.1, we have

$$
\left(\left|\mathcal{J}_{1}\right|+\left|\mathcal{J}_{2}\right|\right) \frac{1}{\sqrt{\epsilon}} \lesssim\|\nabla(\mathbf{u}-\mathbf{H})\|_{L^{\infty}\left(D^{e}\right)} \lesssim \frac{1}{\sqrt{\epsilon}}
$$

We emphasize that $\mathcal{J}_{1}$ and $\mathcal{J}_{2}$ do depend on $\epsilon$. In the next subsection we show that there are some cases such that $1 \lesssim\left|\mathcal{J}_{1}\right|+\left|\mathcal{J}_{2}\right|$ by considering circular inclusions. It implies that $\epsilon^{-1 / 2}$ is the optimal blow-up rate of the gradient.

\subsection{Circular inclusions and optimality of the blow-up rate}

In this subsection we show that $\epsilon^{-1 / 2}$ is a lower bound on the blow-up rate of the gradient by considering two circular inclusions under a uniform loading.

Proposition 6.3. Suppose that $D_{1}$ and $D_{2}$ are disks with the same radius $r_{0}$ and let $\mathbf{u}$ be the solution to (2.10). Let

$$
\alpha^{*}=\alpha^{*}(\lambda, \mu):=\frac{\lambda+\mu}{\mu}
$$


(i) If $\mathbf{H}(x, y)=(A x, B y)^{T}$ with $A \neq 0$, then there are $\epsilon_{0}>0$ and $\alpha_{0}>0$ independent of $\epsilon$ such that for any $(\lambda, \mu)$ satisfying $\alpha^{*}(\lambda, \mu) \leq \alpha_{0}$ and $\epsilon \leq \epsilon_{0}$ the following holds:

$$
1 \lesssim\left|\mathcal{J}_{1}\right| \quad \text { and } \quad \mathcal{J}_{2}=0
$$

(ii) If $\mathbf{H}(x, y)=C(y, x)^{T}$ with $C \neq 0$, then there are $\epsilon_{0}>0$ and $\alpha_{0}>0$ independent of $\epsilon$ such that for any $(\lambda, \mu)$ satisfying $\alpha^{*}(\lambda, \mu) \leq \alpha_{0}$ and $\epsilon \leq \epsilon_{0}$ the following holds:

$$
\mathcal{J}_{1}=0 \quad \text { and } \quad 1 \lesssim\left|\mathcal{J}_{2}\right|
$$

We emphasize that the condition $\alpha^{*}(\lambda, \mu) \leq \alpha_{0}$ can be satisfied even if $\alpha_{0}$ is small. In fact, the strong convexity condition requires $\mu>0$ and $\lambda+\mu>0$. So, by taking negative $\lambda$, the condition is satisfied.

Proof. We only prove (i) since (ii) can be proved similarly.

Since $\mathbf{H}(x, y)=(A x, B y)^{T}$ satisfies $6.2, \mathcal{J}_{2}=0$ by Theorem 6.1 . To prove $1 \lesssim\left|\mathcal{J}_{1}\right|$, we define $\mathbf{r}_{1}$ by

$$
\left(1+\frac{m_{1}}{\sqrt{\epsilon}} t_{1}\right) \mathbf{h}_{1}=\frac{m_{1}}{\sqrt{\epsilon}} \mathbf{q}_{1}+\mathbf{r}_{1}
$$

where $t_{1}$ is the constant appearing in Lemma 3.3. Then $\mathbf{r}_{1}$ satisfies $\mathcal{L}_{\lambda, \mu} \mathbf{r}_{1}=0$ in $D^{e}$ and $\mathbf{r}_{1} \in \mathcal{A}^{*}$. It also satisfies, according to Lemma 3.3 , the boundary condition

$$
\mathbf{r}_{1}=-\frac{m_{1} \alpha_{2} a}{\sqrt{\epsilon} r_{0}^{2}}\left[\begin{array}{l}
x \\
y
\end{array}\right] \quad \text { on } \partial D_{1} \cup \partial D_{2}
$$

Since

$$
\mathcal{J}_{1}=\int_{\partial D^{e}} \partial_{\nu} \mathbf{h}_{1} \cdot \mathbf{H}
$$

we may write, using $6.14,\left(1+\frac{m_{1}}{\sqrt{\epsilon}} t_{1}\right) \mathcal{J}_{1}$ as

$$
\begin{aligned}
\left(1+\frac{m_{1}}{\sqrt{\epsilon}} t_{1}\right) \mathcal{J}_{1} & =\int_{\partial D^{e}} \partial_{\nu}\left(\left(1+\frac{m_{1}}{\sqrt{\epsilon}} t_{1}\right) \mathbf{h}_{1}\right) \cdot \mathbf{H}-\int_{\partial D^{e}} \partial_{\nu} \mathbf{H} \cdot\left(\left(1+\frac{m_{1}}{\sqrt{\epsilon}} t_{1}\right) \mathbf{h}_{1}\right) \\
& =\int_{\partial D^{e}} \partial_{\nu}\left(\frac{m_{1}}{\sqrt{\epsilon}} \mathbf{q}_{1}+\mathbf{r}_{1}\right) \cdot \mathbf{H}-\int_{\partial D^{e}} \partial_{\nu} \mathbf{H} \cdot\left(\frac{m_{1}}{\sqrt{\epsilon}} \mathbf{q}_{1}+\mathbf{r}_{1}\right) \\
& =\frac{m_{1}}{\sqrt{\epsilon}}\left(\int_{\partial D^{e}} \partial_{\nu} \mathbf{q}_{1} \cdot \mathbf{H}-\partial_{\nu} \mathbf{H} \cdot \mathbf{q}_{1}\right)+\int_{\partial D^{e}} \partial_{\nu} \mathbf{r}_{1} \cdot \mathbf{H}-\int_{\partial D^{e}} \partial_{\nu} \mathbf{H} \cdot \mathbf{r}_{1} \\
& =: I_{1}+I_{2}+I_{3} .
\end{aligned}
$$

To estimate $I_{1}$, we first recall that $m_{1}:=\left[\left(\alpha_{1}-\alpha_{2}\right) \sqrt{2\left(\kappa_{1}+\kappa_{2}\right)}\right]^{-1}$. Since $\kappa_{1}=\kappa_{2}=$ $1 / r_{0}$, we have

$$
\frac{m_{1}}{\sqrt{\epsilon}}=\frac{\sqrt{r_{0}}}{2\left(\alpha_{1}-\alpha_{2}\right) \sqrt{\epsilon}} .
$$

Then Green's formula for the Lamé system and 4.48) yield

$$
\begin{aligned}
I_{1} & =\frac{m_{1}}{\sqrt{\epsilon}} \int_{D_{1} \cup D_{2}} \mathcal{L}_{\lambda, \mu} \mathbf{q}_{1} \cdot \mathbf{H} \\
& =\frac{\sqrt{r_{0}}}{2\left(\alpha_{1}-\alpha_{2}\right) \sqrt{\epsilon}}\left[\left(\mathbf{H}\left(\mathbf{p}_{1}\right)-\mathbf{H}\left(\mathbf{p}_{2}\right)\right) \cdot \mathbf{e}_{1}-\sum_{j=1}^{2} \frac{\alpha_{2} a}{\alpha_{1}-\alpha_{2}}\left(\partial_{1} \mathbf{H}\left(\mathbf{p}_{j}\right) \cdot \mathbf{e}_{1}+\partial_{2} \mathbf{H}\left(\mathbf{p}_{j}\right) \cdot \mathbf{e}_{2}\right)\right] .
\end{aligned}
$$


Since $\mathbf{p}_{1}=(-a, 0), \mathbf{p}_{2}=(a, 0)$ and $\mathbf{H}(x, y)=(A x, B y)^{T}$, we arrive at

$$
I_{1}=-\frac{a \sqrt{r_{0}}}{2\left(\alpha_{1}-\alpha_{2}\right) \sqrt{\epsilon}}\left[2 A+\frac{2 \alpha_{2}(A+B)}{\alpha_{1}-\alpha_{2}}\right] .
$$

Since $\alpha_{1}=\frac{\lambda+3 \mu}{4 \pi \mu(\lambda+2 \mu)}$ and $\alpha_{2}=\frac{\lambda+\mu}{4 \pi \mu(\lambda+2 \mu)}$, we have

$$
\begin{aligned}
& \frac{1}{\alpha_{1}-\alpha_{2}}=2 \pi(\lambda+2 \mu)=2 \pi \mu\left(1+\alpha^{*}\right), \\
& \frac{\alpha_{2}}{\alpha_{2}-\alpha_{2}}=\frac{\lambda+\mu}{2 \mu}=\frac{\alpha^{*}}{2} .
\end{aligned}
$$

Since $a=\sqrt{r_{0} \epsilon}+O\left(\epsilon^{3 / 2}\right)$, we have

$$
\frac{a \sqrt{r_{0}}}{\sqrt{\epsilon}}=r_{0}+O(\epsilon)
$$

Substituting 6.19)-6.21 into 6.18, we obtain

$$
\begin{aligned}
I_{1} & =-2 \pi \mu\left(1+\alpha^{*}\right)\left(r_{0}+O(\epsilon)\right)\left[2 A+\alpha^{*}(A+B)\right] \\
& =-2 \pi r_{0} \mu\left(2 A+O\left(\alpha^{*}+\epsilon+\alpha^{*} \epsilon\right)\right) .
\end{aligned}
$$

To estimate $I_{2}$, let $B_{r}$ be the disk of radius $r$ centered at the origin containing $\overline{D_{1} \cup D_{2}}$. Choose $r$ and $R$ so that $r<R$. Let $\chi$ be a smooth radial function such that $\chi(\mathbf{x})=1$ if $|\mathbf{x}| \leq r$ and $\chi(\mathbf{x})=0$ if $|\mathbf{x}| \geq R$. Let $\mathbf{v}:=\chi \mathbf{H}$. Then, using Green's formula, we obtain

$$
\left|I_{2}\right|=\left|\int_{\partial D^{e}} \frac{\partial \mathbf{r}_{1}}{\partial \nu} \cdot \mathbf{v}\right|=\left|\int_{D^{e}} \mathbb{C} \widehat{\nabla} \mathbf{r}_{1}: \widehat{\nabla} \mathbf{v}\right| \leq \mathcal{E}_{D^{e}}\left[\mathbf{r}_{1}\right]^{1 / 2} \mathcal{E}_{D^{e}}[\mathbf{v}]^{1 / 2}
$$

Let $\mathbf{w}(\mathbf{x}):=\chi(\mathbf{x}) \mathbf{x}$ for $\mathbf{x} \in D^{e}$. Then

$$
-\frac{m_{1} \alpha_{2} a}{\sqrt{\epsilon} r_{0}^{2}} \mathbf{w}=\mathbf{r}_{1} \quad \text { on } \partial D^{e}
$$

Then, the variational principle 2.37) yields

$$
\mathcal{E}_{D^{e}}\left[\mathbf{r}_{1}\right] \leq\left(\frac{m_{1} \alpha_{2} a}{\sqrt{\epsilon} r_{0}^{2}}\right)^{2} \mathcal{E}_{D^{e}}[\mathbf{w}] .
$$

We emphasize that the variational principle holds since $\mathbf{r}_{1}$ is a solution of the Lamé system. So, we arrive at

$$
\left|I_{2}\right| \leq \frac{m_{1} \alpha_{2} a}{\sqrt{\epsilon} r_{0}^{2}} \mathcal{E}_{D^{e}}[\mathbf{w}]^{1 / 2} \mathcal{E}_{D^{e}}[\mathbf{v}]^{1 / 2}
$$

Note that $\|\nabla \mathbf{w}\|_{L^{2}\left(D^{e}\right)}^{2} \lesssim 1$ and

$$
\mathcal{E}_{D^{e}}[\mathbf{w}]=\int_{D^{e}} \mathbb{C} \widehat{\nabla} \mathbf{w}: \widehat{\nabla} \mathbf{w} \lesssim(\lambda+2 \mu)\|\nabla \mathbf{w}\|_{L^{2}\left(D^{e}\right)}^{2} \lesssim \lambda+2 \mu .
$$

Here and throughout this proof, $X \lesssim Y$ implies $X \leq C Y$ for some constant $C$ independent of $(\lambda, \mu)$ and $\epsilon$. Similarly, one can see that

$$
\mathcal{E}_{D^{e}}[\mathbf{v}] \lesssim \lambda+2 \mu
$$


So we infer from 6.26 that

$$
\left|I_{2}\right| \lesssim \frac{m_{1} \alpha_{2} a}{\sqrt{\epsilon} r_{0}^{2}}(\lambda+2 \mu) .
$$

It then follows from 6.17), 6.19-6.21 that

$$
\left|I_{2}\right| \lesssim \frac{\alpha_{2}}{\alpha_{1}-\alpha_{2}}(\lambda+2 \mu) \lesssim \alpha^{*} \mu\left(1+\alpha^{*}\right) \lesssim \mu \alpha^{*} .
$$

Since $\mathbf{H}=(A x, B y)$, it is easy to see that

$$
\left|\partial_{\nu} \mathbf{H}\right|_{\partial D^{e}} \mid \lesssim \lambda+2 \mu
$$

So, by 6.15 and the fact that $a \approx \sqrt{\epsilon}$, we see that

$$
\left|I_{3}\right| \lesssim m_{1} \alpha_{2}(\lambda+2 \mu) \lesssim \frac{\alpha_{2}}{\alpha_{1}-\alpha_{2}}(\lambda+2 \mu) \lesssim \mu \alpha^{*} .
$$

Combining 6.16), 6.22), 6.28) and 6.29), we have

$$
\left(1+\frac{m_{1}}{\sqrt{\epsilon}} t_{1}\right) \mathcal{J}_{1}=-4 \pi r_{0} \mu\left(A+O\left(\alpha^{*}+\epsilon+\alpha^{*} \epsilon\right)\right) .
$$

Recall from 3.46 that $\left|t_{j}\right| \lesssim\left(\alpha_{1}+\alpha_{2}\right) \epsilon^{3 / 2}$. So, using 6.20), we have

$$
\left|\frac{m_{1}}{\sqrt{\epsilon}} t_{1}\right| \lesssim \frac{\alpha_{1}+\alpha_{2}}{\alpha_{1}-\alpha_{2}} \epsilon \lesssim\left(1+\frac{2 \alpha_{2}}{\alpha_{1}-\alpha_{2}}\right) \epsilon \lesssim\left(1+\alpha^{*}\right) \epsilon .
$$

Therefore, we finally arrive at

$$
\mathcal{J}_{1}=-\frac{4 \pi r_{0} \mu\left(A+O\left(\alpha^{*}+\epsilon+\alpha^{*} \epsilon\right)\right)}{1+O\left(\epsilon+\alpha^{*} \epsilon\right)} .
$$

Since $A \neq 0$, there are $\alpha_{0}$ and $\epsilon_{0}$ such that

$$
1 \lesssim\left|\mathcal{J}_{1}\right|
$$

for all $(\lambda, \mu)$ satisfying $\alpha^{*} \leq \alpha_{0}$ and $\epsilon \leq \epsilon_{0}$. This completes the proof.

Corollary 6.2 and Proposition 6.3 shows that $\epsilon^{-1 / 2}$ is a lower bound on $\nabla \mathbf{u}$ as $\epsilon \rightarrow 0$. In fact, we have

$$
\|\nabla \mathbf{u}\|_{L^{\infty}\left(B \backslash\left(D_{1} \cup D_{2}\right)\right)} \approx \epsilon^{-1 / 2}
$$

as $\epsilon \rightarrow 0$, provided that $\alpha^{*} \leq \alpha_{0}$. In fact, we have a more refined estimate for $\nabla \mathbf{u}(0,0)$.

Theorem 6.4. Let $\mathbf{u}$ be the solution to 2.10) when $D_{1}$ and $D_{2}$ are disks with the same radius. Suppose that the Lamé parameters $(\lambda, \mu)$ satisfy $\alpha^{*} \leq \alpha_{0}$. Then the following holds as $\epsilon \rightarrow 0$.

(i) If $\mathbf{H}(x, y)=(A x, B y)$ with $A \neq 0$, then

$$
\left|\partial_{1} u_{1}(0,0)\right| \approx \epsilon^{-1 / 2} \quad \text { and } \quad\left|\partial_{2} u_{1}(0,0)\right|+\left|\partial_{1} u_{2}(0,0)\right|+\left|\partial_{2} u_{2}(0,0)\right| \lesssim 1 .
$$


(ii) If $\mathbf{H}(x, y)=C(y, x)$ with $C \neq 0$, then

$$
\left|\partial_{1} u_{2}(0,0)\right| \approx \epsilon^{-1 / 2} \quad \text { and } \quad\left|\partial_{1} u_{1}(0,0)\right|+\left|\partial_{2} u_{1}(0,0)\right|+\left|\partial_{2} u_{2}(0,0)\right| \lesssim 1 .
$$

Proof. Suppose that $\mathbf{H}(x, y)=(A x, B y)$ with $A \neq 0$. It then follows from 6.4 and 6.12) that

$$
\nabla(\mathbf{u}-\mathbf{H})(\mathbf{x})=\nabla \mathbf{b}(\mathbf{x})+\mathcal{J}_{1} \nabla \mathbf{q}_{1}(\mathbf{x}) .
$$

Then (3.56), 6.5) and 6.12 yield 6.31). (ii) can be proved similarly.

The estimates (6.31) and 6.32) yield, in particular,

$$
|\nabla \cdot \mathbf{u}(0,0)| \approx \epsilon^{-1 / 2} \quad \text { and } \quad\left|\partial_{2} u_{1}(0,0)\right|+\left|\partial_{1} u_{2}(0,0)\right| \lesssim 1
$$

if $\mathbf{H}(x, y)=(A x, B y)$ with $A \neq 0$, and

$$
\left|\partial_{1} u_{2}(0,0)\right|+\left|\partial_{2} u_{1}(0,0)\right| \approx \epsilon^{-1 / 2} \text { and }|\nabla \cdot \mathbf{u}(0,0)| \lesssim 1
$$

if $\mathbf{H}(x, y)=C(y, x)$ with $C \neq 0$. Note that $\nabla \cdot \mathbf{u}$ represents the bulk force while $\left|\partial_{1} u_{2}\right|+$ $\left|\partial_{2} u_{1}\right|$ the magnitude of the shear force. These estimates are in accordance with results of numerical experiments in [19].

\section{Conclusion}

We investigate the problem of characterizing the stress concentration in the narrow region between two hard inclusions and deriving optimal estimates of the magnitude of the stress in the context of the isotropic linear elasticity. We introduce singular functions which are constructed using nuclei of strain, and then show that they capture precisely the singular behavior of the stress as the distance between two inclusions tends to zero. As consequences we are able to derive an upper bound of the blow-up rate of the stress, namely, $\epsilon^{-1 / 2}$ where $\epsilon$ is the distance between two inclusions. We then show that $\epsilon^{-1 / 2}$ is an optimal blow-up rate in the sense that it is also a lower bound on the rate of the stress blow-up in some cases. We show that it is a lower bound in the case when inclusions are disks of the same radii.

To show that $\epsilon^{-1 / 2}$ is a lower bound in the case of circular inclusions, we impose a certain condition on the Lamé parameters. This condition does not seem natural and may be removed. In fact, it is likely, as suggested in numerical experiments in [19], that $\epsilon^{-1 / 2}$ is a lower bound without any assumption on Lamé parameters if the background field is a uniform loading. It is quite interesting and challenging to clarify this.

\section{A The Neumann-Poincaré operator and the exterior prob- lem}

In this section we prove Propositions 2.1 and 2.2, and Theorem 2.5. The proofs are based on the layer potential technique. 


\section{A.1 The NP operator}

Let us begin by reviewing well-known results on the layer potentials on simple closed curves. Let $D$ be a simply connected bounded domain in $\mathbb{R}^{2}$ with the $C^{1, \alpha}(\alpha>0)$ smooth boundary. The co-normal derivative of the single layer potential and the double layer potential satisfy the following jump formulas:

$$
\begin{aligned}
& \left.\partial_{\nu} \mathcal{S}_{\partial D}[\boldsymbol{\varphi}]\right|_{ \pm}(\mathbf{x})=\left( \pm \frac{1}{2} I+\mathcal{K}_{\partial D}^{*}\right)[\boldsymbol{\varphi}](x), \quad \mathbf{x} \in \partial D, \\
& \left.\mathcal{D}_{\partial D}[\boldsymbol{\varphi}]\right|_{ \pm}(\mathbf{x})=\left(\mp \frac{1}{2} I+\mathcal{K}_{\partial D}\right)[\boldsymbol{\varphi}](x), \quad \mathbf{x} \in \partial D,
\end{aligned}
$$

where $\mathcal{K}_{\partial D}$ is the boundary integral operator defined by

$$
\mathcal{K}_{\partial D}[\boldsymbol{\varphi}](x):=\text { p.v. } \int_{\partial D} \partial_{\nu_{\mathbf{y}}} \boldsymbol{\Gamma}(\mathbf{x}-\mathbf{y}) \boldsymbol{\varphi}(\mathbf{y}) d \sigma(\mathbf{y}), \quad \mathbf{x} \in \partial D,
$$

and $\mathcal{K}_{\partial D}^{*}$ is the adjoint operator of $\mathcal{K}_{\partial D}$ on $L^{2}(\partial D)^{2}$. Here, p.v. stands for the Cauchy pricincipal value. The operators $\mathcal{K}_{\partial D}$ and $\mathcal{K}_{\partial D}^{*}$ are called the Neumann-Poincaré (NP) operators.

It is known that the operator $-1 / 2 I+\mathcal{K}_{\partial D}^{*}$ is a Fredholm operator of index 0 , it is invertible on $H^{-1 / 2}(\partial D)^{2}$, and its kernel is of three dimensions (see, for example, [14]). It is worth mentioning that the NP operator can be realized as a self-adjoint operator on $H^{-1 / 2}(\partial D)^{2}$ by introducing a new inner product, and it is polynomially compact (see [6]).

We now consider $D^{e}=\mathbb{R}^{2} \backslash \overline{\left(D_{1} \cup D_{2}\right)}$, whose boundary $\partial D^{e}$ consists of two disjoint curves $\partial D_{1}$ and $\partial D_{2}$. To define the NP operator in this case, we consider the solution to 2.10 in the form of 2.19 , namely,

$$
\mathbf{u}(\mathbf{x})=\mathbf{H}(\mathbf{x})+\mathcal{S}_{\partial D_{1}}\left[\boldsymbol{\varphi}_{1}\right](\mathbf{x})+\mathcal{S}_{\partial D_{2}}\left[\boldsymbol{\varphi}_{2}\right](\mathbf{x}) .
$$

The boundary condition on $\partial D^{e}$ in $(2.10)$ amounts to

$$
\left.\partial_{\nu}\left(\mathbf{H}+\mathcal{S}_{\partial D_{1}}\left[\boldsymbol{\varphi}_{1}\right]+\mathcal{S}_{\partial D_{2}}\left[\boldsymbol{\varphi}_{2}\right]\right)\right|_{-}=0 \quad \text { on } \partial D^{e},
$$

which, according to A.1, is equivalent to the following system of integral equations:

$$
\left\{\begin{array}{l}
\left(-\frac{1}{2} I+\mathcal{K}_{\partial D_{1}}^{*}\right)\left[\boldsymbol{\varphi}_{1}\right]+\left.\partial_{\nu} \mathcal{S}_{\partial D_{2}}\left[\boldsymbol{\varphi}_{2}\right]\right|_{\partial D_{1}}=-\partial_{\nu} \mathbf{H} \quad \text { on } \partial D_{1} \\
\left.\partial_{\nu} \mathcal{S}_{\partial D_{1}}\left[\boldsymbol{\varphi}_{1}\right]\right|_{\partial D_{2}}+\left(-\frac{1}{2} I+\mathcal{K}_{\partial D_{2}}^{*}\right)\left[\boldsymbol{\varphi}_{2}\right]=-\partial_{\nu} \mathbf{H} \quad \text { on } \partial D_{2}
\end{array}\right.
$$

This system of integral equations can be rewritten as

$$
\left(-\frac{1}{2} \mathbb{I}+\mathbb{K}^{*}\right)\left[\begin{array}{l}
\boldsymbol{\varphi}_{1} \\
\boldsymbol{\varphi}_{2}
\end{array}\right]=-\left[\begin{array}{c}
\left.\partial_{\nu} \mathbf{H}\right|_{\partial D_{1}} \\
\left.\partial_{\nu} \mathbf{H}\right|_{\partial D_{2}}
\end{array}\right]
$$

where $\mathbb{I}$ is the identity operator and $\mathbb{K}^{*}$, which is the NP operator on $\partial D^{e}$, is defined by

$$
\mathbb{K}^{*}:=\left[\begin{array}{cc}
\mathcal{K}_{\partial D_{1}}^{*} & \partial_{\nu_{1}} \mathcal{S}_{\partial D_{2}} \\
\partial_{\nu_{2}} \mathcal{S}_{\partial D_{1}} & \mathcal{K}_{\partial D_{2}}^{*}
\end{array}\right]
$$


A special attention is necessary for the off-diagonal entries in the above: For example, $\partial_{\nu_{1}} \mathcal{S}_{\partial D_{2}}$ means that the single layer potential is defined on $\partial D_{2}$ and the co-normal derivative is evaluated on $\partial D_{1}$, so the operator maps $H^{-1 / 2}\left(\partial D_{2}\right)^{2}$ into $H^{-1 / 2}\left(\partial D_{1}\right)^{2}$. One can see that the adjoint operator $\mathbb{K}$ of $\mathbb{K}^{*}$ on $L^{2}\left(\partial D^{e}\right)^{2}$ is given by

$$
\mathbb{K}=\left[\begin{array}{cc}
\mathcal{K}_{\partial D_{1}} & \left.\mathcal{D}_{\partial D_{2}}\right|_{\partial D_{1}} \\
\left.\mathcal{D}_{\partial D_{1}}\right|_{\partial D_{2}} & \mathcal{K}_{\partial D_{2}}
\end{array}\right]
$$

Here $\left.\mathcal{D}_{\partial D_{2}}\right|_{\partial D_{1}}$ means the double layer potential on $\partial D_{2}$ evaluated on $\partial D_{1}$. We emphasize that

$$
\left.\left(\mathcal{D}_{\partial D_{1}}\left[\boldsymbol{\varphi}_{1}\right]+\mathcal{D}_{\partial D_{2}}\left[\boldsymbol{\varphi}_{2}\right]\right)\right|_{+}=\left(-\frac{1}{2} \mathbb{I}+\mathbb{K}\right)\left[\begin{array}{l}
\boldsymbol{\varphi}_{1} \\
\boldsymbol{\varphi}_{2}
\end{array}\right] \quad \text { on } \partial D^{e}
$$

Lemma A.1. The operator $-1 / 2 \mathbb{I}+\mathbb{K}^{*}$ is of Fredholm index 0 on $H^{-1 / 2}\left(\partial D^{e}\right)^{2}$.

Proof. We express $-1 / 2 \mathbb{I}+\mathbb{K}^{*}$ as

$$
-1 / 2 \mathbb{I}+\mathbb{K}^{*}=\left[\begin{array}{cc}
-1 / 2 I+\mathcal{K}_{\partial D_{1}}^{*} & 0 \\
0 & -1 / 2 I+\mathcal{K}_{\partial D_{2}}^{*}
\end{array}\right]+\left[\begin{array}{cc}
0 & \partial_{\nu_{1}} \mathcal{S}_{\partial D_{2}} \\
\partial_{\nu_{2}} \mathcal{S}_{\partial D_{1}} & 0
\end{array}\right] .
$$

Since $-1 / 2 I+\mathcal{K}_{\partial D_{j}}^{*}$ is of Fredholm index 0 for $j=1,2$, so is the first operator on the righthand side above. Since $\partial D_{1}$ and $\partial D_{2}$ are apart, the second operator on the right-hand side is compact. Since the Fredholm index is invariant under a compact perturbation, $-1 / 2 \mathbb{I}+\mathbb{K}^{*}$ is of Fredholm index 0 .

In the following we prove Propositions 2.1 and 2.2, and Theorem 2.5. We prove Proposition 2.2 first since it is simpler.

\section{A.2 Proof of Proposition 2.2}

We first prove the following lemma.

Proposition A.2. The operator $-1 / 2 \mathbb{I}+\mathbb{K}^{*}$ is invertible on $H_{\Psi}^{-1 / 2}\left(\partial D_{1}\right) \times H_{\Psi}^{-1 / 2}\left(\partial D_{2}\right)$.

Proof. As we see from $(\mathrm{A} .8)$ that $-1 / 2 \mathbb{I}+\mathbb{K}^{*}$ is a compact perturbation of an operator which is invertible on $H_{\Psi}^{-1 / 2}\left(\partial D_{1}\right) \times H_{\Psi}^{-1 / 2}\left(\partial D_{2}\right)$. So by the Fredholm alternative it suffices to prove the injectivity of $-1 / 2 \mathbb{I}+\mathbb{K}^{*}$.

Suppose that

$$
\left(-\frac{1}{2} \mathbb{I}+\mathbb{K}^{*}\right)\left[\begin{array}{l}
\varphi_{1} \\
\varphi_{2}
\end{array}\right]=0
$$

for some $\left(\boldsymbol{\varphi}_{1}, \boldsymbol{\varphi}_{2}\right) \in H_{\Psi}^{-1 / 2}\left(\partial D_{1}\right) \times H_{\Psi}^{-1 / 2}\left(\partial D_{2}\right)$ and let

$$
\mathbf{u}(\mathbf{x}):=\mathcal{S}_{\partial D_{1}}\left[\boldsymbol{\varphi}_{1}\right](\mathbf{x})+\mathcal{S}_{\partial D_{2}}\left[\boldsymbol{\varphi}_{2}\right](\mathbf{x}), \quad \mathbf{x} \in \mathbb{R}^{2} .
$$

Then A.9 implies that $\mathcal{L}_{\lambda, \mu} \mathbf{u}=0$ in $D_{i}$ and $\partial_{\nu} \mathbf{u}=0$ on $\partial D_{i}$ for $i=1,2$. So

$$
\mathbf{u}=\sum_{j=1}^{3} a_{i j} \Psi_{j} \quad \text { in } D_{i}
$$


for some constants $a_{i j}$. Since the single layer potential is continuous across $\partial D_{i}$, we have $\left.\mathbf{u}\right|_{+}=\sum_{j=1}^{3} a_{i j} \Psi_{j}$ on $\partial D_{i}$. Moreover, by the jump formula A.1 for the single layer potential, we have

$$
\left.\int_{\partial D_{i}} \partial_{\nu} \mathbf{u}\right|_{+} \cdot \Psi_{j}=\int_{\partial D_{i}}\left(\boldsymbol{\varphi}_{i}+\left.\partial_{\nu} \mathbf{u}\right|_{-}\right) \cdot \Psi_{j}=\int_{\partial D_{i}} \boldsymbol{\varphi}_{i} \cdot \Psi_{j}=0
$$

since $\varphi_{i} \in H_{\Psi}^{-1 / 2}\left(\partial D_{i}\right)$. So $\mathbf{u}$ is a solution to 2.10 with $\mathbf{H}=0$. It is worth mentioning that the decay condition at $\infty$ is satisfies because $\varphi_{j} \in H_{\Psi}^{-1 / 2}\left(\partial D_{j}\right)$. We then have

$$
\int_{D^{e}} \mathbb{C} \widehat{\nabla} \mathbf{u}: \widehat{\nabla} \mathbf{u}=\left.\int_{\partial D^{e}} \partial_{\nu} \mathbf{u}\right|_{+} \cdot \mathbf{u}=\sum_{j=1}^{3} \sum_{i=1}^{2} a_{i j} \int_{\partial D_{i}} \partial_{\nu} \mathbf{u} \cdot \Psi_{j}=0,
$$

where the last equality follows from (2.7). Hence $\mathbf{u}=0$ in $D^{e}$. By the jump formula A.1 for the single layer potential, we have

$$
\boldsymbol{\varphi}_{j}=\left.\partial_{\nu} \mathbf{u}\right|_{+}-\left.\partial_{\nu} \mathbf{u}\right|_{-}=0 \quad \text { on } \partial D_{i}
$$

for $i=1,2$. This completes the proof.

Proof of Proposition 2.2. Note that since $\mathcal{L}_{\lambda, \mu} \mathbf{H}=0$ in $\mathbb{R}^{2},\left.\partial_{\nu} \mathbf{H}\right|_{\partial D_{i}} \in H_{\Psi}^{-1 / 2}\left(\partial D_{i}\right)$ for $i=1,2$. So we solve A.4) for $\left(\boldsymbol{\varphi}_{1}, \boldsymbol{\varphi}_{2}\right)$ on $H_{\Psi}^{-1 / 2}\left(\partial D_{1}\right) \times H_{\Psi}^{-1 / 2}\left(\partial D_{2}\right)$. Then $\mathbf{u}$ defined by 2.19 is the solution to 2.10 .

\section{A.3 Proof of Proposition 2.1}

Let $\mathbf{u}$ be the solution to (2.6) and let $\mathbf{f}:=\partial_{\nu} \mathbf{u}$ on $\partial \Omega$. Let $\mathbf{H}_{\Omega}$ be the function defined by (2.17). We emphasize that $\mathbf{H}_{\Omega}(\mathbf{x})$ is defined not only for $\mathbf{x} \in \Omega$, but also for $\mathbf{x} \in \mathbb{R}^{2} \backslash \bar{\Omega}$. Moreover, one can see from A.1 and A.2 that the following holds:

$$
\left.\mathbf{H}_{\Omega}\right|_{-}-\left.\mathbf{H}_{\Omega}\right|_{+}=\mathbf{g},\left.\quad \partial_{\nu} \mathbf{H}_{\Omega}\right|_{-}-\left.\partial_{\nu} \mathbf{H}_{\Omega}\right|_{+}=\mathbf{f} \quad \text { on } \partial \Omega .
$$

Let $\left(\boldsymbol{\varphi}_{1}, \boldsymbol{\varphi}_{2}\right) \in H_{\Psi}^{-1 / 2}\left(\partial D_{1}\right) \times H_{\Psi}^{-1 / 2}\left(\partial D_{2}\right)$ be the unique solution to A.4 with $\mathbf{H}$ replaced by $\mathbf{H}_{\Omega}$, and let

$$
\mathbf{v}_{1}(\mathbf{x})=\mathbf{H}_{\Omega}(\mathbf{x})+\mathcal{S}_{\partial D_{1}}\left[\boldsymbol{\varphi}_{1}\right](\mathbf{x})+\mathcal{S}_{\partial D_{2}}\left[\boldsymbol{\varphi}_{2}\right](\mathbf{x}), \quad \mathbf{x} \in D^{e} \backslash \partial \Omega .
$$

Then $\mathbf{v}_{1}$ is a solution to

$$
\begin{cases}\mathcal{L}_{\lambda, \mu} \mathbf{v}=0 & \text { in } D^{e} \backslash \partial \Omega \\ \mathbf{v}=\sum_{j=1}^{3} a_{i j} \Psi_{j}(\mathbf{x}), & \text { on } \partial D_{i}, \quad i=1,2 \\ \left.\mathbf{v}\right|_{-}-\left.\mathbf{v}\right|_{+}=\mathbf{g},\left.\quad \partial_{\nu} \mathbf{v}\right|_{-}-\left.\partial_{\nu} \mathbf{v}\right|_{+}=\mathbf{f} & \text { on } \partial \Omega, \\ \mathbf{v}(\mathbf{x})=O\left(|\mathbf{x}|^{-1}\right) & \text { as }|\mathbf{x}| \rightarrow \infty\end{cases}
$$

where the constants $a_{i j}$ are determined by the condition (2.7). 
Let

$$
\mathbf{v}_{2}(\mathbf{x}):= \begin{cases}\mathbf{u}(\mathbf{x}) & \mathbf{x} \in \Omega \backslash \overline{D_{1} \cup D_{2}} \\ 0 & \mathbf{x} \in \mathbb{R}^{2} \backslash \bar{\Omega}\end{cases}
$$

Then $\mathbf{v}_{2}$ is also a solution to A.13) with the same $\mathbf{g}$ and $\mathbf{f}$.

Let $\mathbf{v}:=\mathbf{v}_{1}-\mathbf{v}_{2}$. Then $\mathbf{v}$ is a solution to A.13 with $\mathbf{g}=0$ and $\mathbf{f}=0$. So, we have

$$
\int_{D^{e}} \mathbb{C} \widehat{\nabla} \mathbf{v}: \hat{\nabla} \mathbf{v}=\left.\int_{\partial D^{e}} \partial_{\nu} \mathbf{v}\right|_{+} \cdot \mathbf{v}=\sum_{j=1}^{3} \sum_{i=1}^{2} c_{i j} \int_{\partial D_{i}} \partial_{\nu} \mathbf{u} \cdot \Psi_{j}=0,
$$

where the last equality follows from (2.7). So we infer $\mathbf{v}=0$ in $D^{e}$. In particular, $\mathbf{u}=\mathbf{v}_{1}$ in $\Omega \backslash \overline{D_{1} \cup D_{2}}$ as desired.

\section{A.4 Proof of Theorem 2.5}

Let

$$
V=\left\{\mathbf{f} \in H^{1 / 2}\left(\partial D^{e}\right)^{2}: \mathbb{K}[\mathbf{f}]=\frac{1}{2} \mathbf{f}\right\}
$$

and

$$
W=\left\{\mathbf{f} \in H^{-1 / 2}\left(\partial D^{e}\right): \mathbb{K}^{*}[\mathbf{f}]=\frac{1}{2} \mathbf{f}\right\},
$$

which are null spaces of $-1 / 2 \mathbb{I}+\mathbb{K}$ and $-1 / 2 \mathbb{I}+\mathbb{K}^{*}$, respectively. In particular, we have $\operatorname{dim} V=\operatorname{dim} W$. For $j=1,2,3$, let

$$
\alpha_{j}^{1}(\mathbf{x})= \begin{cases}\Psi_{j}(\mathbf{x}) & \text { if } \mathbf{x} \in \partial D_{1} \\ 0 & \text { if } \mathbf{x} \in \partial D_{2}\end{cases}
$$

and

$$
\alpha_{j}^{2}(\mathbf{x})= \begin{cases}0 & \text { if } \mathbf{x} \in \partial D_{1}, \\ \Psi_{j}(\mathbf{x}) & \text { if } \mathbf{x} \in \partial D_{2} .\end{cases}
$$

Lemma A.3. The following holds:

(i) $\operatorname{dim} V=\operatorname{dim} W=6$.

(ii) $\left\{\alpha_{j}^{1}, \alpha_{j}^{2}: j=1,2,3\right\}$ is a basis of $V$.

Proof. If $\mathbf{x} \in \mathbb{R}^{2} \backslash \overline{D_{i}}$, then

$$
\mathcal{D}_{\partial D_{i}}\left[\Psi_{j}\right](\mathbf{x})=\int_{D_{i}} \mathbb{C} \widehat{\nabla}_{\mathbf{y}} \boldsymbol{\Gamma}(\mathbf{x}-\mathbf{y}): \widehat{\nabla} \Psi_{j}(\mathbf{y})=0,
$$

for $i=1,2$ and $j=1,2,3$. In particular, we have $\left(-1 / 2 I+\mathcal{K}_{\partial D_{i}}\right)\left[\Psi_{j}\right]=0$ on $\partial D_{i}$. So, we infer that $\left\{\alpha_{j}^{1}, \alpha_{j}^{2}\right\} \subset V$. Since $\alpha_{j}^{1}$ and $\alpha_{j}^{2}, j=1,2,3$, are linearly independent, we infer $\operatorname{dim} V \geq 6$.

On the other hand, since $-1 / 2 \mathbb{I}+\mathbb{K}^{*}$ is a Fredholm operator of index 0 , we have $H^{-1 / 2}\left(\partial D^{e}\right)^{2}=$ Range $\left(-1 / 2 \mathbb{I}+\mathbb{K}^{*}\right) \oplus W$. According to Proposition A.2, $H_{\Psi}^{-1 / 2}\left(\partial D_{1}\right) \times$ $H_{\Psi}^{-1 / 2}\left(\partial D_{2}\right) \subset \operatorname{Range}\left(-1 / 2 \mathbb{I}+\mathbb{K}^{*}\right)$. Since $H_{\Psi}^{-1 / 2}\left(\partial D_{i}\right)^{2}$ has co-dimension 3 in $H^{-1 / 2}\left(\partial D_{i}\right)$. we infer that the co-dimension of Range $\left(-1 / 2 \mathbb{I}+\mathbb{K}^{*}\right)$ in $H^{-1 / 2}\left(\partial D^{e}\right)^{2}$ is larger than or equals to 6 . So, $\operatorname{dim} W \leq 6$. This completes the proof. 
Lemma A.4. Let

$$
W_{\Psi}:=\left\{\mathbf{f}=\left(\mathbf{f}_{1}, \mathbf{f}_{2}\right) \in W: \int_{\partial D_{1}} \mathbf{f}_{1} \cdot \Psi_{j}+\int_{\partial D_{2}} \mathbf{f}_{2} \cdot \Psi_{j}=0 \text { for } j=1,2,3\right\} .
$$

Then, $\operatorname{dim} W_{\Psi}=3$.

Proof. For $i=1,2$, define $\beta_{j}^{i}$ by

$$
\beta_{j}^{i}:= \begin{cases}\alpha_{j}^{i} & \text { if } j=1,2, \\ \alpha_{3}^{i}+c_{i} \alpha_{1}^{i}+d_{i} \alpha_{2}^{i} & \text { if } j=3,\end{cases}
$$

where the constants $c_{i}$ and $d_{i}$ are chosen so that

$$
\left\langle\beta_{j}^{i}, \beta_{l}^{k}\right\rangle=0 \quad \text { if }(i, j) \neq(k, l) .
$$

Then there is an eigenfunction $\mathbf{f}_{j}^{i} \in W$ such that

$$
\left\langle\mathbf{f}_{j}^{i}, \beta_{l}^{k}\right\rangle= \begin{cases}1 & \text { if }(i, j)=(k, l), \\ 0 & \text { if }(i, j) \neq(k, l) .\end{cases}
$$

In fact, since $\left(-1 / 2 \mathbb{I}+\mathbb{K}^{*}\right)\left[\beta_{j}^{i}\right] \in H_{\Psi}^{-1 / 2}\left(\partial D_{1}\right) \times H_{\Psi}^{-1 / 2}\left(\partial D_{2}\right)$, there is a unique $\mathbf{g}=$ $\left(\mathbf{g}_{1}, \mathbf{g}_{2}\right) \in H_{\Psi}^{-1 / 2}\left(\partial D_{1}\right) \times H_{\Psi}^{-1 / 2}\left(\partial D_{2}\right)$ such that

$$
\left(-\frac{1}{2} \mathbb{I}+\mathbb{K}^{*}\right)[\mathbf{g}]=\left(-\frac{1}{2} \mathbb{I}+\mathbb{K}^{*}\right)\left[\beta_{j}^{i}\right]
$$

Let $\mathbf{f}:=\beta_{j}^{i}-\mathbf{g}$. Then, $\mathbf{f} \in W$. Moreover, we have

$$
\left\langle\mathbf{f}, \beta_{l}^{k}\right\rangle=\left\langle\beta_{j}^{i}, \beta_{l}^{k}\right\rangle
$$

So $\mathbf{f}_{j}^{i}:=\left\langle\beta_{j}^{i}, \beta_{j}^{i}\right\rangle^{-1} \mathbf{f}$ is the desired function.

Let for $i=1,2$

$$
\mathbf{g}_{1}^{i}:=\mathbf{f}_{1}^{i}-c^{i} \mathbf{f}_{3}^{i}, \quad \mathbf{g}_{2}^{i}:=\mathbf{f}_{2}^{i}-d^{i} \mathbf{f}_{3}^{i}, \quad \mathbf{g}_{3}^{i}:=\mathbf{f}_{3}^{i} .
$$

Then, one can see that

$$
\left\langle\mathbf{g}_{j}^{i}, \alpha_{l}^{k}\right\rangle= \begin{cases}1 & \text { if }(i, j)=(k, l), \\ 0 & \text { if }(i, j) \neq(k, l) .\end{cases}
$$

Then $\mathbf{g}_{j}^{1}-\mathbf{g}_{j}^{2}(j=1,2,3)$ three linearly independent functions belonging to $W_{\Psi}$, while $\mathbf{g}_{j}^{1}+\mathbf{g}_{j}^{2}(j=1,2,3)$ does not belong to $W_{\Psi}$. So $\operatorname{dim} W_{\Psi}=3$.

Define the operator $\mathbb{S}: H^{-1 / 2}\left(\partial D^{e}\right)^{2} \rightarrow H^{1 / 2}\left(\partial D^{e}\right)^{2}$ as follows: for $\mathbf{f}=\left(\mathbf{f}_{1}, \mathbf{f}_{2}\right) \in$ $H^{-1 / 2}\left(\partial D^{e}\right)$ let

$$
\mathbf{v}(\mathbf{x}):=\mathcal{S}_{\partial D_{1}}\left[\mathbf{f}_{1}\right](\mathbf{x})+\mathcal{S}_{\partial D_{2}}\left[\mathbf{f}_{2}\right](\mathbf{x})
$$

and

$$
\mathbb{S}[\mathbf{f}]:=\left[\begin{array}{c}
\left.\mathbf{v}\right|_{\partial D_{1}} \\
\left.\mathbf{v}\right|_{\partial D_{2}}
\end{array}\right]
$$


Lemma A.5. Let

$$
V^{+}:=\operatorname{span}\left\{\Psi_{1}, \Psi_{2}, \Psi_{3}\right\}
$$

Then, the following holds:

(i) $\mathbb{S}$ maps $W$ into $V$, and $\mathbb{S}$ is injective on $W_{\Psi}$.

(ii) $V=\mathbb{S}\left(W_{\Psi}\right) \oplus V^{+}$.

Proof. Let $\mathbf{f} \in W$ and define $\mathbf{v}$ by A.20. Then we have

$$
\left.\partial_{\nu} \mathbf{v}\right|_{-}=\left(-1 / 2 \mathbb{I}+\mathbb{K}^{*}\right)[\mathbf{f}]=0 \quad \text { on } \partial D^{e} .
$$

Since $\mathcal{L}_{\lambda, \mu} \mathbf{v}=0$ in $D_{i}(i=1,2)$, we infer that $\mathbf{v}=\sum_{j} a_{i j} \Psi_{j}$ on $\partial D_{i}$ for some constants $a_{i j}$. So $\mathbb{S}[\mathbf{f}] \in V$.

If further $\mathbf{f} \in W_{\Psi}$, then $\mathbf{v}(\mathbf{x})=O\left(|\mathbf{x}|^{-1}\right)$ as $|\mathbf{x}| \rightarrow 0$. So, if $\mathbb{S}[\mathbf{f}]=0$, then $\mathbf{v}=0$ in $D^{e}$, and hence $\mathbf{v}=0$ in $\mathbb{R}^{2}$. Thus we have $\mathbf{f}=\left.\partial_{\nu} \mathbf{v}\right|_{+}-\left.\partial_{\nu} \mathbf{v}\right|_{-}=0$ on $\partial D^{e}$. This proves (i).

We now show that $\mathbb{S}\left(W_{\Psi}\right) \cap V^{+}=\{0\}$. In fact, if $\mathbf{f}=\left(\mathbf{f}_{1}, \mathbf{f}_{2}\right) \in W_{\Psi}$ satisfies $\mathbb{S}[\mathbf{f}]=$ $\sum_{j} a_{j} \Psi_{j}$ on $\partial D^{e}$, let

$$
\mathbf{v}(\mathbf{x}):=\mathcal{S}_{\partial D_{1}}\left[\mathbf{f}_{1}\right](\mathbf{x})+\mathcal{S}_{\partial D_{2}}\left[\mathbf{f}_{2}\right](\mathbf{x}), \quad \mathbf{x} \in \mathbb{R}^{2}
$$

Then $\mathbf{v} \in \mathcal{A}$, and

$$
\begin{aligned}
\int_{D^{e}} \mathbb{C} \widehat{\nabla} \mathbf{v}: \widehat{\nabla} \mathbf{v} & =-\left.\int_{\partial D^{e}} \partial_{\nu} \mathbf{v}\right|_{+} \cdot \mathbf{v} \\
& =-\int_{\partial D^{e}} \mathbf{f} \cdot\left(\sum_{j} a_{j} \Psi_{j}\right)-\left.\int_{\partial D^{e}} \partial_{\nu} \mathbf{v}\right|_{-} \cdot\left(\sum_{j} a_{j} \Psi_{j}\right)=0 .
\end{aligned}
$$

So, $\mathbf{v}=0$ in $D^{e}$, and hence $\sum_{j} a_{j} \Psi_{j}=0$ on $\partial D^{e}$.

Since $\mathbb{S}$ is injective on $W_{\Psi}, \operatorname{dim} \mathbb{S}\left(W_{\Psi}\right)=3$. So $\operatorname{dim} \mathbb{S}\left(W_{\Psi}\right) \oplus V^{+}=6$. This yields (ii).

Since $-1 / 2 \mathbb{I}+\mathbb{K}$ is fredholm, we have $H^{1 / 2}\left(\partial D^{e}\right)^{2}=\operatorname{Range}(-1 / 2 \mathbb{I}+\mathbb{K}) \oplus V^{+}$. So we obtain the following proposition.

Proposition A.6. $H^{1 / 2}\left(\partial D^{e}\right)^{2}=\operatorname{Range}(-1 / 2 \mathbb{I}+\mathbb{K}) \oplus \mathbb{S}\left(W_{\Psi}\right) \oplus V^{+}$.

Proof of Theorem 2.5. Let $\mathbf{g} \in H^{1 / 2}\left(\partial D^{e}\right)^{2}$. According to the previous proposition, there is $\mathbf{f}=\left(\mathbf{f}_{1}, \mathbf{f}_{2}\right) \in H^{1 / 2}\left(\partial D^{e}\right)^{2}, \boldsymbol{\varphi}=\left(\boldsymbol{\varphi}_{1}, \boldsymbol{\varphi}_{2}\right) \in W_{\Psi}$, and constants $a_{1}, a_{2}, a_{3}$ such that

$$
\mathbf{g}=\left(-\frac{1}{2} \mathbb{I}+\mathbb{K}\right)[\mathbf{f}]+\mathbb{S}[\boldsymbol{\varphi}]+\sum_{j=1}^{3} a_{j} \Psi_{j}
$$

Then the solution $\mathbf{u}$ is given by

$$
\mathbf{u}=\sum_{i=1}^{2}\left(\mathcal{D}_{\partial D_{i}}\left[\mathbf{f}_{i}\right]+\mathcal{S}_{\partial D_{i}}\left[\boldsymbol{\varphi}_{i}\right]\right)+\sum_{j=1}^{3} a_{j} \Psi_{j} \text { in } D^{e}
$$

Note that $\sum_{i=1}^{2}\left(\mathcal{D}_{\partial D_{i}}\left[\mathbf{f}_{i}\right]+\mathcal{S}_{\partial D_{i}}\left[\boldsymbol{\varphi}_{i}\right]\right) \in \mathcal{A}$ by Lemma 2.6. So $\mathbf{u} \in \mathcal{A}^{*}$. 
For uniqueness, assume that $\mathbf{u}$ and $\mathbf{v}$ are two solutions in $\mathcal{A}^{*}$, and let $\mathbf{w}:=\mathbf{u}-\mathbf{v}$. Then $\mathbf{w} \in \mathcal{A}^{*}$ and $\mathbf{w}=0$ on $\partial D^{e}$. Let $\mathbf{w}=\mathbf{w}_{1}+\mathbf{w}_{2}$ be such that $\mathbf{w}_{1} \in \mathcal{A}$ and $\mathbf{w}_{2}=\sum_{j=1}^{3} a_{j} \Psi_{j}$. Then by Lemma 2.8, we have

$$
0=\int_{D^{e}} \mathbb{C} \widehat{\nabla} \mathbf{w}: \widehat{\nabla} \mathbf{w}=\int_{D^{e}} \mathbb{C} \widehat{\nabla} \mathbf{w}_{1}: \widehat{\nabla} \mathbf{w}_{1}
$$

Since $\mathbf{w}_{1}(\mathbf{x}) \rightarrow 0$ as $|\mathbf{x}| \rightarrow \infty, \mathbf{w}_{1}=0$ in $D^{e}$. So, $\sum_{j=1}^{3} a_{j} \Psi_{j}=0$ on $\partial D^{e}$, which implies $a_{j}=0, j=1,2,3$. This completes the proof.

\section{References}

[1] H. Ammari, G. Ciraolo, H. Kang, H. Lee and K. Yun, Spectral analysis of the Neumann-Poincaré operator and characterization of the stress concentration in antiplane elasticity, Arch. Rational Mech. Anal., 208 (2013), 275-304.

[2] H. Ammari and H. Kang, Polarization and moment tensors with applications to inverse problems and effective medium theory, Applied Mathematical Sciences, Vol. 162, Springer-Verlag, New York, 2007.

[3] H. Ammari, H. Kang, H. Lee, J. Lee and M. Lim, Optimal bounds on the gradient of solutions to conductivity problems, J. Math. Pure. Appl. 88 (2007), 307-324.

[4] H. Ammari, H. Kang, H. Lee, M. Lim and H. Zribi, Decomposition theorems and fine estimates for electrical fields in the presence of closely located circular inclusions, J. Differ. Equations 247 (2009), 2897-2912.

[5] H. Ammari, H. Kang and M. Lim, Gradient estimates for solutions to the conductivity problem, Math. Ann. 332(2) (2005), 277-286.

[6] K. Ando, Y. Ji, H. Kang, K. Kim and S. Yu, Spectral properties of the NeumannPoincaré operator and cloaking by anomalous localized resonance for the elasto-static system, Euro. J. Appl. Math., to appear, arXiv:1510.00989.

[7] I. Babuška, B. Andersson, P. Smith and K. Levin, Damage analysis of fiber composites. I. Statistical analysis on fiber scale, Comput. Methods Appl. Mech. Engrg. 172 (1999), 27-77.

[8] E.S. Bao, Y. Li and B. Yin, Gradient estimates for the perfect conductivity problem, Arch. Rational Mech. Anal. 193 (2009), 195-226.

[9] J. Bao, H. Li and Y. Li, Gradient estimates for solutions of the Lamé system with partially infinite coefficients, Arch. Rational Mech. Anal. 215 (2015), 307-351.

[10] J. Bao, H. Li and Y. Li, Gradient estimates for solutions of the Lamé system with partially infinite coefficients in dimensions greater than two, Adv. Math. 305 (2017), $298-338$.

[11] E.S. Bao, Y. Li and B. Yin, Gradient estimates for the perfect and insulated conductivity problems with multiple inclusions, Commun. Part. Diff. Eq. 35 (2010), 19822006. 
[12] L. Berlyand and A. Kolpakov, Network approximation in the limit of small interparticle distance of the effective properties of a high-contrast random dispersed composite, Arch. Rational Mech. Anal. 159 (2001), 179-227.

[13] C. Constanda, The boundary integral equation method in plane elasticity. Proc. Am. Math. Soc. 123 (1995), 3385-3396.

[14] B.E.J. Dahlberg, C.E. Kenig and G.C. Verchota. Boundary value problems for the systems of elastostatics in Lipschitz domains, Duke Math. J. 57(3) (1988), 795-818.

[15] H. Dong and H. Li, Optimal estimates for the conductivity problem by Green's function method, arXiv:1606.02793.

[16] J. E. Flaherty and J. B. Keller, Elastic behavior of composite media, Comm. Pure. Appl. Math. 26 (1973), 565-580.

[17] Y. Gorb, Singular behavior of electric field of high contrast concentrated composites, SIAM Multiscale Model. Simul. 13 (2015), 1312-1326.

[18] Y. Gorb and A. Novikov, Blow-up of solutions to a $p$-Laplace equation, SIAM Multiscale Model. Simul. 10 (2012), 727-743.

[19] H. Kang and E. Kim, Estimation of stress in the presence of closely located elastic inclusions: A numerical study, Contemporary Math. 660 (2016), 45-57.

[20] H. Kang, H. Lee and K. Yun, Optimal estimates and asymptotics for the stress concentration between closely located stiff inclusions, Math. Ann. 363 (2015), 12811306.

[21] H. Kang, M. Lim and K. Yun, Asymptotics and computation of the solution to the conductivity equation in the presence of adjacent inclusions with extreme conductivities, J. Math. Pure. Appl. 99 (2013), 234-249.

[22] H. Kang, M. Lim and K. Yun, Characterization of the electric field concentration between two adjacent spherical perfect conductors, SIAM J. Appl. Math. 74 (2014), $125-146$.

[23] H. Kang and S. Yu, A proof of the Flaherty-Keller formula on the effective property of densely packed elastic composites, arXiv:1707.02205.

[24] H. Kang and K. Yun, Optimal estimates of the field enhancement in presence of a bowtie structure of perfectly conducting inclusions in two dimensions, arXiv:1707.00098.

[25] J. B. Keller, Conductivity of a medium containing a dense array of perfectly conducting spheres or cylinders or nonconducting cylinders, J. Appl. Phys. 34 (1963), 991-993.

[26] J. Lekner, Electrostatics of two charged conducting spheres, Proc. R. Soc. A, 468 (2012), 2829-2848.

[27] H. Li, Y. Li, E.S. Bao and B. Yin, Derivative estimates of solutions of elliptic systems in narrow domains, Q. Appl. Math. 72 (2014), 589-596. 
[28] Y.Y. Li and L. Nirenberg, Estimates for elliptic system from composite material, Comm. Pure Appl. Math., LVI (2003), 892-925.

[29] M. Lim and S. Yu, Asymptotics of the solution to the conductivity equation in the presence of adjacent circular inclusions with finite conductivities, J. Math. Anal. Appl. 421 (2015), 131-156.

[30] M. Lim and S. Yu, Stress concentration for two nearly touching circular holes, arXiv:1705.10400.

[31] M. Lim and K. Yun, Blow-up of electric fields between closely spaced spherical perfect conductors, Commun. Part. Diff. Eq. 34 (2009), 1287-1315.

[32] M. Lim and K. Yun, Strong influence of a small fiber on shear stress in fiber-reinforced composites, J. Differ. Equations 250 (2011), 2402-2439.

[33] A. E. H. Love, A treatise on the mathematical theory of elasticity, 4th edition, Cambridge University Press, Cambridge (1927).

[34] S. Yu and H. Ammari, Plasmonic interaction between nanosphere, SIAM Review, to appear, arXiv:1604.02624.

[35] K. Yun, Estimates for electric fields blown up between closely adjacent conductors with arbitrary shape, SIAM J. Appl. Math. 67 (2007), 714-730.

[36] K. Yun, Optimal bound on high stresses occurring between stiff fibers with arbitrary shaped cross sections, J. Math. Anal. Appl. 350 (2009), 306-312.

[37] K. Yun, An optimal estimate for electric fields on the shortest line segment between two spherical insulators in three dimensions, J. Differ. Equations 261 (2016), 148-188. 